\title{
Attention modulation of complex motion patterns in human visual cortex
}

\author{
Dissertation \\ for the award of the degree \\ "Doctor of Philosophy" \\ Division of Mathematics and Natural Sciences \\ of the Georg-August-Universität Göttingen
}

submitted by

Sepideh Fazeli Neishabour

from Mashhad-Iran

Göttingen, 2014 


\section{Thesis Committee}

Prof. Dr. Stefan Treue (Advisor, First Referee)

Cognitive Neuroscience Laboratory

German Primate Center (DPZ)

Kellnerweg 4

37077 Göttingen Germany

PD Dr. Peter Dechent (Second Referee)

Göttingen University Medical School

Dept. of Cognitive Neurology

Robert-Koch-Str. 40

37075 Göttingen Germany

Prof. Dr. Alexander Gail

Sensorimotor Neuroscience Laboratory

German Primate Center (DPZ)

Center Kellnerweg 4

37077 Göttingen Germany

Prof. Dr. Fred Wolf

Max Planck Institute for Dynamics and Self-Organization Theoretical Neurophysics

Am Fassberg 17

37077 Göttingen Germany

\section{Members of the Examination Board}

Prof. Dr. Hansjörg Scherberger

Neurobiology Laboratory

German Primate Center (DPZ)

Kellnerweg 4

37077 Göttingen Germany

Prof. Dr. Ralf Heinrich

Dept. Cellular Neurobiology

Schwann-Schleiden Research Centre

Julia-Lermontowa-Weg 3

37077 Göttingen Germany

Date of the oral examination: 30 July 2014 
Herewith I declare that I have written this thesis independently and with no other aids and sources than quoted.

Sepideh Fazeli Neishabour

Göttingen, 27. May 2014 
To my mother 
- What would you think it's worth telling future generations about the life you've lived and the lessons you've learned from it?

- "I should like to say two things, one intellectual and one moral. The intellectual thing I should want to say is this: When you are studying any matter, or considering any philosophy, ask yourself only what are the facts and what is the truth that the facts bear out. Never let yourself be diverted either by what you wish to believe, or by what you think would have beneficent social effects if it were believed. But look only, and solely, at what are the facts. That is the intellectual thing that I should wish to say.

The moral thing I should wish to say... I should say love is wise, hatred is foolish. In this world which is getting more closely and closely interconnected we have to learn to tolerate each other, we have to learn to put up with the fact that some people say things that we don't like. We can only live together in that way and if we are to live together and not die together we must learn a kind of charity and a kind of tolerance which is absolutely vital to the continuation of human life on this planet."

BBC's Face to Face interview of Bertrand Russell 


\section{Acknowledgements}

First of all, I would like to express my special thanks of gratitude to Professor Stefan Treue and PD.Dr. Peter Dechent, my research supervisors, who gave me the opportunity to do my $\mathrm{PhD}$ project on my field of interest under their supervision. Over the last years, I have gained a lot from their patient guidance, enthusiastic encouragement and useful critiques of this research work. I thank Professor Alexander Gail and Professor Fred Wolf, members of my thesis committee for their continuous supports throughout my PhD work. I thank Professor Hansjörg Scherberger and Professor Ralf Heinrich for their kind support in evaluating this thesis. I would like to thank Professor Melanie Wilke as the head of the cognitive neurology department of university medicine Göttingen for her spiritual and financial support to conduct the fMRI part of my PhD project there.

I would also like to thank Dr Carsten Schmidt-Samoa for his irreplaceable advice and assistance on my fMRI project. I thank Ralf Brockhausen, Kevin Windolph, Severin Heumüller, Nina Käding and specially Ilona Pfahlert and Britta Perl for their valuable support throughout this research. And special thanks goes to Beatrix Glaser for her reliable and kind administrative support.

I thank Luis Ramos for his help in collecting psychophysical data. I am thankful to my coleagues: Bahareh Taghizadeh, Vera Veith, Philipp Schwedhelm, Dr. Suresh Krishna and specially Amr Maamoun for their kind help in proof-reading of my thesis and Dr. Cliodhna Quigley, Janina Hüer and again Vera Veith for providing great assistance on my psychophysics project. I would also like to thank all my present and the past lab members in cognitive neuroscience lab and cognitive neurology department for making the workday more enjoyable.

Finally, I wish to thank my mother for her support and encouragement throughout my life and my friends who helped me in finishing this project.

Thanks again to all who helped me. Without their help I wouldn't be able to make it. 


\section{Table of Contents}

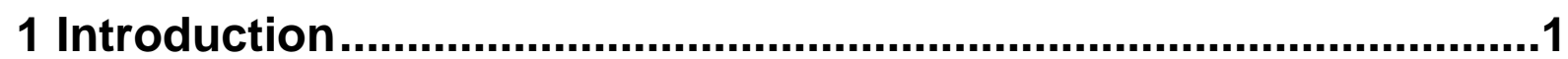

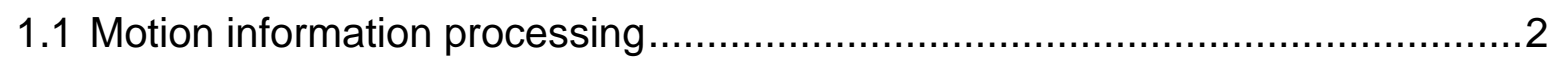

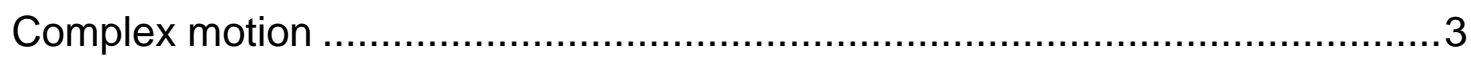

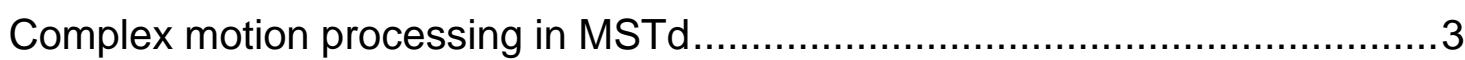

Inferior satellite of MT-complex as the human homologue for MSTd ...............4

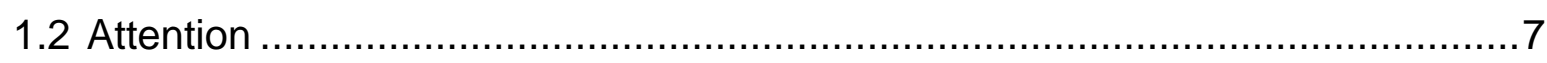

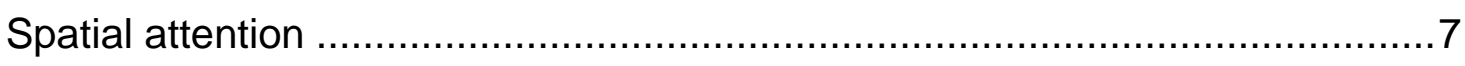

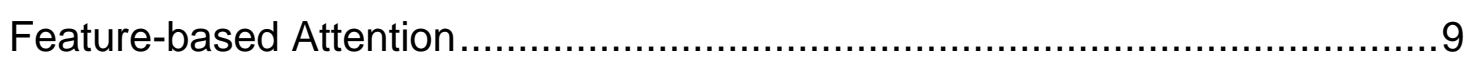

2 Original manuscripts ................................................................17

2.1 Attentional modulation of human BOLD responses to spiral motion patterns ..19

2.2 The influence of spatial attention on human direction discrimination

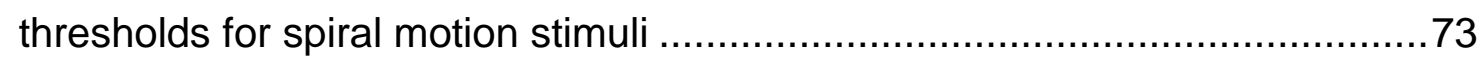

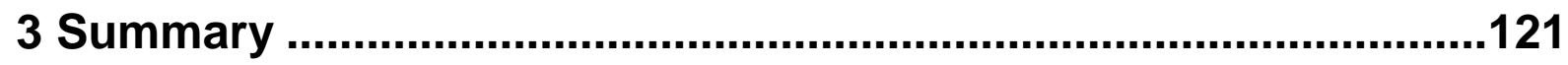

Bibliography.............................................................................123

Curriculum Vitae ...........................................................................133 



\section{Introduction}

When we are moving through the environment, our visual system exposes to a barrage of motion information about the objects around us as well as our own heading. Processing such an amount of incoming sensory data is beyond the power of the available processing resources. Thus, our visual system is provided with several mechanisms to select the most relevant information. Selective visual attention is one key solution to filter out the incoming visual information based on their relevance for the behavior. Visual attention emphasizes the representation of the behaviorally significant stimuli by dedicating the limited visual processing resources to them at the expense of the irrelevant information. Visual motion information is getting processed in a hierarchy of visual areas termed as the "dorsal stream", which starts in primary visual cortex (area V1) and ends in parietal lobe. Among the visual areas in the dorsal stream, middle temporal area (MT) and medial superior temporal area (MST) have the key role in visual motion processing for their preponderance of motion-sensitive neurons.

In this PhD dissertation, I studied the influence of attention on processing of complex motion information in human visual system. I used so called "spiral motion" patterns as the probe stimuli to simulate complex motion patterns. In the second chapter, I investigated the neuronal correlates of attention in response to "spiral" stimuli using functional magnetic resonance imaging (fMRI). I assessed the tuning as well as the influence of attention on such a hypothetical tuning to spiral motions in MT and MST areas in the dorsal stream of the human visual cortex, in addition to examining the influence of attention on the hemodynamic responses to spiral motion patterns. In the third chapter, I investigated the behavioral correlates of graded attention in discriminating spiral motions in a human psychophysics study.

In this chapter, I aimed to provide the reader with a comprehensive introduction about the particularly important concepts for this study. I first introduced the processing of visual motion with a particular emphasize on complex motion processing. Then, the cortical area MST, which has a key role in complex motion processing, was extensively assessed. I also granted a broad review of attentional mechanism involved in visual information processing. 


\subsection{Motion information processing}

Visual incoming information enters the visual system through the eyes. In retina, the light sensitive cells code the visual information. These neuronal codes are relayed further to the lateral geniculate nucleus (LGN) in thalamus. In the next stage, visual information goes to the primary visual cortex (area V1) through LGN. Information processing in visual cortex is carrying out in two distinct pathways known as the "dorsal stream" and the "ventral stream". They are both originating from area $V 1$ in the occipital lobe of the brain. The ventral stream travels to the temporal lobe via area V2, V3, V4, posterior inferior temporal (PIT) as well as central inferior temporal area (CIT). Ventral stream is mostly involved in object recognition and therefore it is termed as the "what" pathway. The dorsal stream, known as "where" pathway, ends in parietal lobe via area V2, V3, middle temporal area (MT), medial superior temporal area (MST), lateral intraparietal area (LIP) as well as ventral intraparietal area (VIP), (for review see: Maunsell and Newsome, 1987). The dorsal stream is involved in processing of motion information in order to guide actions in addition to developing spatial awareness. Therefore, deficits of the dorsal stream by either cerebral lesions (Newsome and Paré, 1988; Zeki, 1991) or brain stimulation (Becker et al., 2013) could cause motion blindness. Moreover, it is widely accepted that the motion processing along the dorsal stream is accomplishing in a hierarchical fashion (Maunsell and Newsome, 1987; Van Essen and Maunsell, 1983). Within this hierarchy, area V1 as the first area is characterized with orientation selectivity and small receptive fields (Hubel and Wiesel, 1968). As the next stage, medial temporal area (MT or V5) gets its input directly from V1 (Maunsell and Van Essen, 1983a; Zeki, 1974). MT cells have bigger receptive field compared to V1 (Dubner and Zeki, 1971) and large proportion of them show tuning (could be described by Gaussian function) to the direction of translation (linear) motion (Albright, 1984; Dubner and Zeki, 1971; Maunsell and Van Essen, 1983b). Going higher along the hierarchy, MST receives most of its input from MT (Maunsell and Van Essen, 1983a; Ungerleider and Desimone, 1986). The receptive field size of MST neurons is bigger than V1 and MT, mostly extends into the ipsilateral visual field (Albright and Desimone, 1987; Desimone and Ungerleider, 1986; Duffy and Wurtz, 1991a; Komatsu and Wurtz, 1988a; Lagae et al., 1994; Tanaka and Saito, 1989). Moreover, its dorsal part (MSTd) demonstrated selectivity to complex motions such as expansion, rotation 
(Duffy and Wurtz, 1991a, b) as well as spiral motion patterns (Spiral patterns are one class of optic flow patterns. They are uniquely determined with their pitch according to their radii, where expansion has the angle of $0^{\circ}$ )(Graziano et al., 1994). There are two remarkable trends in hierarchical organization of the dorsal stream. First, the size of the neuronal receptive field is bigger for the higher areas along the hierarchy. Second, the preferred stimulus of the neurons gets more complex at each stage of the visual hierarchy (Van Essen and Maunsell, 1983).

In the second chapter, I investigated the attentional modulation of hemodynamic responses to spiral motion pattern, and hence focused on area MT and MST in the dorsal stream.

\section{Complex motion}

When we move through the environment, projection of the objects in the visual field into our retina forms a complex motion patterns termed as "optic flow". These complex motion patterns are carrying essential information about the self-motion, heading as well as surrounding moving objects and therefore could develop our perception of the three-dimensional visual space in order to guide and control the actions.

Several psychophysics studies investigated the complex motion perception in human visual system. It was shown that the detection threshold is lower for expanding (radial) and rotating moving patterns than translation (Freeman and Harris, 1992). Another study by (Steiner et al., 1994) showed that the interocular transfer is higher for expansion/rotation motion-after-effect than the translation. In the same line, (Takeuchi, 1997) suggested an asymmetry in processing of expanding versus contracting moving patterns by conducting a visual search task. All these studies suggest the existence of a dedicated cerebral circuitry for processing complex motion patterns selectively. Moreover, using a sub-threshold summation experiment (Meese and Anderson, 2002) indicated the need for a selective mechanism to spiral motion patterns than cardinal directions of radial and circular motions.

\section{Complex motion processing in MSTd}

In order to find the neuronal substrate for complex motion perception, many monkey electrophysiological studies investigated the characteristic of the dorsal part of MST (MSTd) because it is getting its input from MT (MT is tuned to 
translation motion). Summarizing the most leading studies, it has been demonstrated that neurons in area MSTd have large receptive fields, most of them extending into the ipsilateral visual field (Duffy and Wurtz, 1991a), which makes MSTd to best response to large visual stimuli like optic flow patterns. Neurons in MSTd showed preference for stimuli containing speed gradient (Duffy and Wurtz, 1997), which could be produced in retina by the relative movement of solid objects. MSTd cells showed position variant responses to the large stimuli with large shift, which is an essential characteristic for heading determination (Duffy and Wurtz, 1995). Moreover, MSTd neurons are responding to pursuit eye movement (Komatsu and Wurtz, 1988b), which is relating producing of complex motion in retina to the direction of gaze.

All theses mentioned characteristic of MSTd in addition to its selectivity to complex motion patterns, make MST a good candidate for processing complex motion patterns.

\section{Inferior satellite of MT-complex as the human homologue for MSTd}

Human MT-complex is relatively large cluster mostly located on the lateral region of the occipital lobe within the inferior temporal sulcus (ITS), its ascending limb or its posterior extension. The MT-complex is mostly involved in motion processing as a homologue for clusters of motion-sensitive areas in monkey's dorsal superior temporal sulcus (STS). The most common localizer to identify MT-complex is the contrast of moving versus stationary dots, however, this simple contrast falls short in distinguishing human analogs of monkey MT and its surrounding satellites such as FST, MSTv and MSTd. Fig.1 illustrated the schematic of MT-complex and its surrounding regions in human and monkey brains. The first human imaging studies (Tootell and Taylor, 1995; Zeki et al., 1991) demonstrated motion sensitivity in human MT-complex. Following these studies, the MT and MST subdivision of MT-complex was subdivided based on their receptive field size and their retinotopic organization (Huk et al., 2002). They identified MT as a cluster with only contralateral activation and retinotopic organization, and MST as an area, exhibiting both ipsi and contralateral activation, without a remarkable retinotopic organization. Cerebral activity related to optic flow perception was identified in inferior part of MT-complex (de Jong et al., 1994) and another study 
(Morrone et al., 2000) demonstrated that the inferior region of MT-complex responds specifically to complex motion pattern (expansion and rotation).

Another study by (Peuskens et al., 2001) demonstrated that attending to heading stimulus (optic flow with speed gradient) versus dimming peripheral dot (while viewing optic flow pattern) causes modulation in inferior satellite of MT-complex. Considering all these finding, the inferior district of MT-complex might be a potential candidate corresponding to monkey MSTd, however there is continuing debate on identifying human homologue of monkey MSTd (opponent conclusion: (Tootell et al., 1998).

In the second chapter, we examined the voxel-based tunings to spiral motion patterns in MT and MST subdivisions of human MT-complex. 

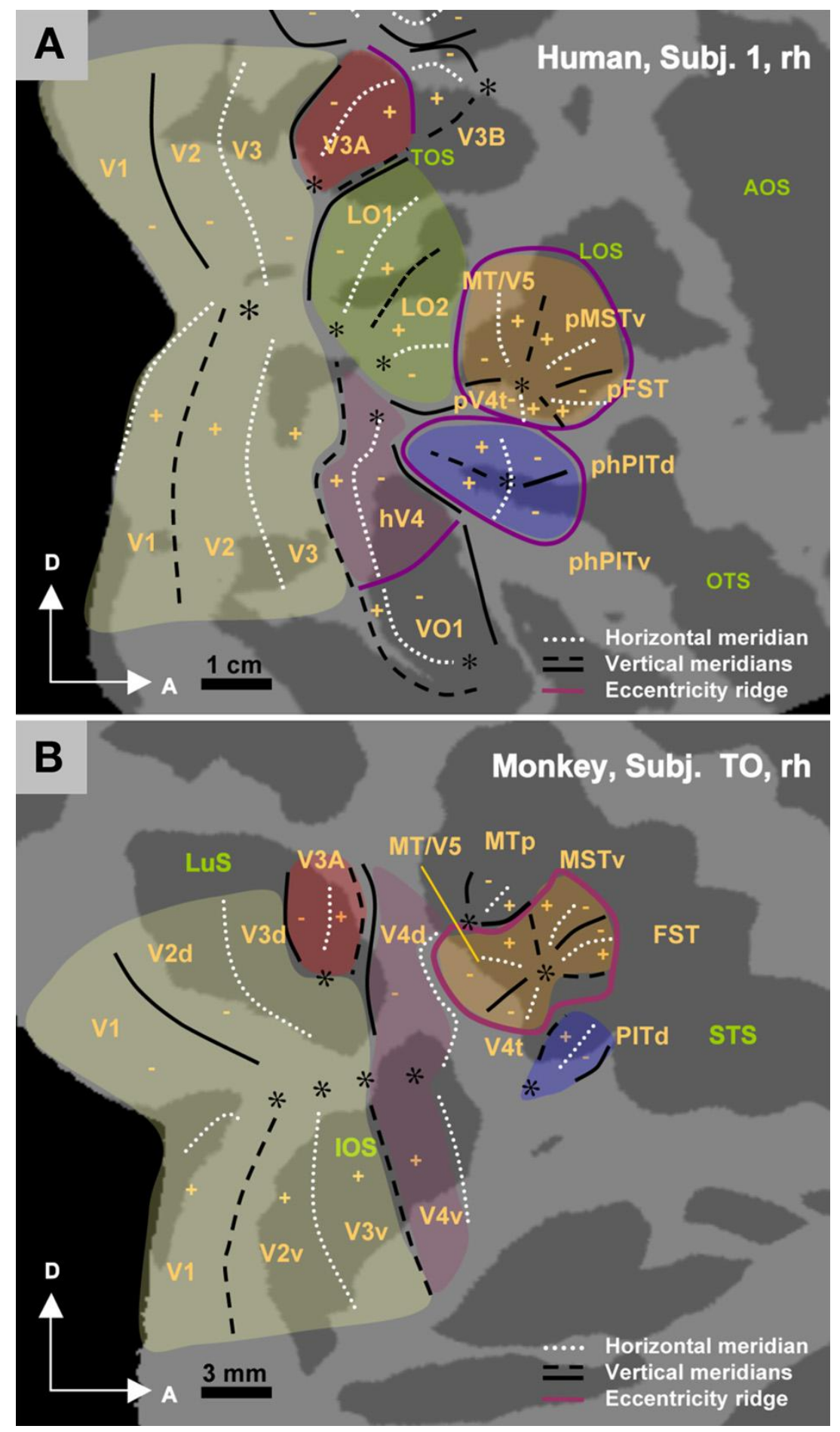

Figure 1: Schematic representation of the MT/V5 cluster and neighboring areas. A: Human data (Kolster et al., 2010) B: Monkey data (Kolster et al., 2009).

Kolster, H., R. Peeters and G. A. Orban (2010). "The retinotopic organization of the human middle temporal area MT/V5 and its cortical neighbors." JNS 30(29): 9801-9820.

Reprinted by permission from Journal of Neuroscience 


\subsection{Attention}

Although a notable part of primate's brain is dedicated to visual processing, the huge amount of incoming information to visual system is beyond its available recourses. This challenge demonstrates the necessity of a selective mechanism to filter out the behaviorally relevant information in expense of others. Dedication of the highest concentration of cone cells in retina to the foveal vision, accompanied by fast saccades to explore the visual space is one solution to select out the relevant information. In addition, visual attention is another mechanism for filtering out the relevant information based on their importance for the behavior. Visual attention can be either stimulus driven (bottom-up) or goal directed (top-down). As an example for top-down attention, if we have prior information about the color and size of a particular book beside its approximate position in a library bookshelf, we could deploy our attention only to the books with the same features as the target book, and therefore we can find it quite faster. Attention allocation accompanied with eye movement is known as overt attention, whereas, if it comes without any eye movement, it is termed as covert attention. Attention selects visual information according to their position in the visual filed (spatial attention), or their attribution to a specific feature such as color or motion direction (feature-based attention). Moreover, attention can also be directed to a complex objects (object-based attention), toward a moment in time (temporal attention) or particular sensory modality (multisensory attention).

\section{Spatial attention}

It is not exaggeration to say that spatial attention is the most intensively studied form of attention. Over the last three decades, many of studies focused to address its behavioral and neuronal correlates. The pioneering psychophysics studies of covert spatial attention showed that directing the spotlight of attention toward targets in the visual field, enhances their perception by improving the performance at them, reducing the reaction time with the cost of the impaired performance at the distracters (Eriksen and Yeh, 1985; Posner, 1980; Yeshurun and Carrasco, 1998). It was also shown that spreading attention over a larger field compared to the focused one, reduce the spatial resolution and performance efficiency (Eriksen 
and St. James, 1986; Eriksen and Yeh, 1985), which indicated the limitation of attention resources.

The achieved improvements by spatial attention at behavioral level motivated scientists to investigate its neuronal correlate. The first monkey electrophysiological study by (Moran and Desimone, 1985) showed that firing rate of neurons in area V4 increase if the probe stimulus was shown at the attended location versus un-attended one. Most of the following electrophysiological studies examined the effect of covert spatial attention by comparing the neuronal responses when attention is directed into versus outside their receptive fields in the same sensory configuration. Using this paradigm, spatial attention was tested in many visual cortical areas such as V1, V2, V4 (Luck et al., 1997) V1, V4 (McAdams and Maunsell, 1999) and MT, MST (Treue and Maunsell, 1996), all reporting that directing spatial attention from outside to the neuron's receptive field modulates its firing rate. Comparing determined attentional modulation in V1-V4 (McAdams and Maunsell, 1999), MT-MST (Treue and Maunsell, 1996), MT-VIP (Cook and Maunsell, 2002), we could conclude that the order of spatial attention modulation is bigger in higher areas along the visual hierarchy. Moreover, studying attentional modulation in almost all extrastriate visual areas yielded consistent findings, that attention modulation of sensory information is stimulus selective, i.e. attentional effect depends on the feature of the sensory inputs (Treue, 2001). Investigating spatial attention in area V4 (McAdams and Maunsell, 1999) and MT, MST (Treue and Maunsell, 1996) demonstrated it as a multiplicative effect. It means that responses of neurons tuned to any feature of the stimulus are enhanced by a constant gain across the feature dimension, i.e. the dynamic range (amplitude) of their tuning function is scaled up without any effect on its selectivity (bandwidth). Fig.2 depicts the multiplicative modulation observed in MT. In addition to the attention modulation of single neurons firing rates, it was shown that attention increases the gamma band $(35-90 \mathrm{~Hz})$ synchronization of V4 population cells (Fries et al., 2001).

Beside the electrophysiological animal studies, neuronal basis of spatial attention has been investigated in human visual cortex using neuroimaging techniques. It was shown that spatial attention increases the BOLD signal in the retinotopic visual areas representing the attended location (O'Craven and Savoy, 1995; Somers et al., 1999) and suppresses it in the areas representing the unattended 
locations (Kastner et al., 1998; Smith et al., 2000). The attention modulation was also observed even without direct visual stimulation (Kastner et al., 1999). Similar effect of spatial attention was also detected in subcortical regions, including lateral geniculate nucleus (LGN) in thalamus (O'Connor et al., 2002). Recently several fMRI studies (Saproo and Serences, 2010; Serences et al., 2009) reported the modulation of the orientation population codes with spatial attention using voxelbased tuning functions as well as multi voxel pattern analysis. Moreover, it was shown that spatial attention improves the ecoding of orientation (Kamitani and Tong, 2005) as well as linear motion direction (Kamitani and Tong, 2006).
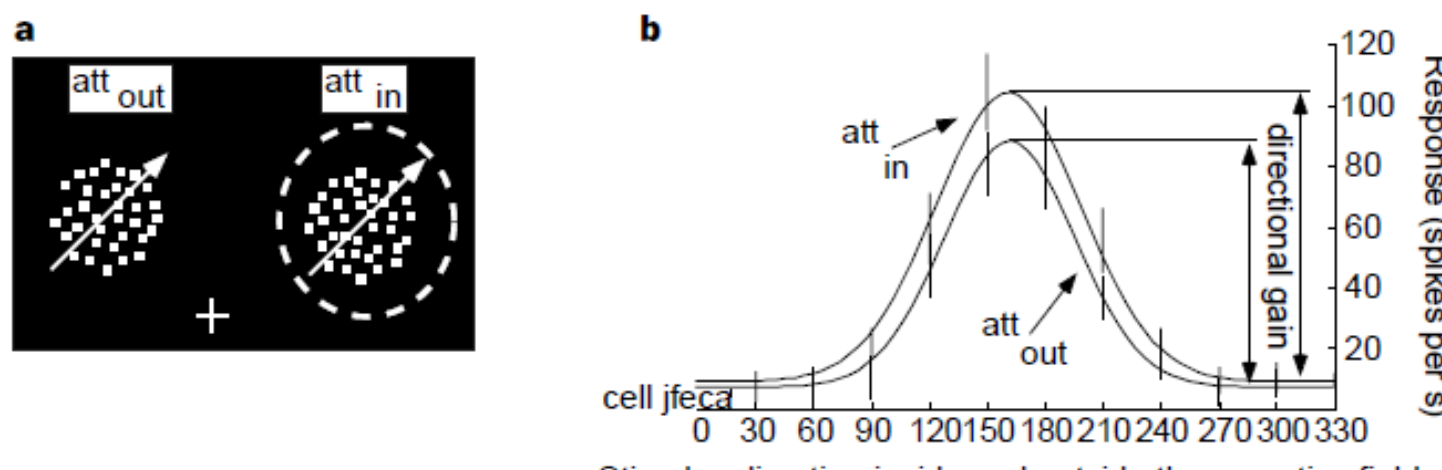

Figure 2: Effect of spatial attention on the directional tuning curve. A: Experimental paradigm: two identical random dot patterns (RDP) were displayed inside the receptive field indicated with the dashed circle, as well as outside the receptive filed at the ipsilateral hemifield. Both RDPs had the same direction randomly picked from twelve possible directions. B: directional tuning curves in different attentional conditions. The upper tuning curve was determined when attention was to the RDP inside the receptive field (marked att $_{\text {in }}$ ), and the lower tuning curve was extracted when attention was to the RDP outside the receptive field (marked att ${ }_{\text {out }}$ ). Spatial attention modulates the tuning curves by scaling up the amplitude without any effect on the tuning width (multiplicative modulation).

Treue, S. and J. C. Martinez-Trujillo (1999). "Feature-based attention influences motion processing gain in macaque visual cortex." N 399(6736): 575-579.

Reprinted by permission from Nature

\section{Feature-based Attention}

Majority of researches in studying attention concentrated on investigating the mechanism of the spatial attention, i.e. selecting relevant visual information from a specific region in the visual field. However, in some circumstances such as visual search, we might need to select important information across the entire visual field. In such conditions, knowing the exact features of a visual target (such as its 
color), makes it easier to find it among many distracters. For example, when we are searching for a specific car in a big parking lot, knowing its color is a significant cue to find it faster. It is because attention can be deployed to the target features in order to select them across the visual field.

Investigating the neuronal and behavioral correlates of the feature-based attention was the foci of many psychophysical, electrophysiological as well as neuroimaging studies. A pioneering electrophysiological study by Treue and Martinez-Trujillo (1999) reported the effect of feature-based attention on MT neurons firing rate. In this study, two stimuli were presented to a monkey; one inside and the other one outside the neuron's receptive field. The inside stimulus contained the preferred direction the outside one had either preferred or null direction. They showed when spatial attention was at the outsided stimulus with the preferred direction, the firing rate of the MT cell was higher compared to when attention was at the null direction outside the receptive field. The experimental paradigm as well as their result depicts in Fig. 3.

Moreover, they investigated the effect of feature-based attention on the neuronal tuning curve. They showed that feature-based attention affects the gain of the tuning curve without changing its bandwidth. They observed that this gain modulation was depended on the similarity between the attended feature and preferred feature of the neuron. Thus, they proposed "feature-similarity gain" model for feature-based attention. Later, in a follow up electrophysiological study (Martinez-Trujillo and Treue, 2004), they showed that feature-based attention increases the firing rate of neurons preferring the attended feature, and suppresses the activity of those tuned to the opposing feature, which shown in Fig.4. Thus, feature-based attention resulted to an increased of the population responses selectivity in a non-multiplicative way. In other words, feature-based attention enhances the representation of the attended feature at the population level. In the same line, feature-based attention was assessed in human psychophysics studies (Liu and Hou, 2011; Liu and Mance, 2011; Saenz et al., 2003; Shih and Sperling, 1996). As an example, Saenz et al., (2003) studied feature-based attention using a dual task paradigm with two spatially distant stimuli at the left and right visual field. Subjects were tasked to detect a speed change on both sides. They saw subjects had better performance when the attended stimuli shared the same feature (either upward or downward, or same 
color of either green or red) compared to when they had opposing features, which provided another support for the "feature-similarity gain" model.

a

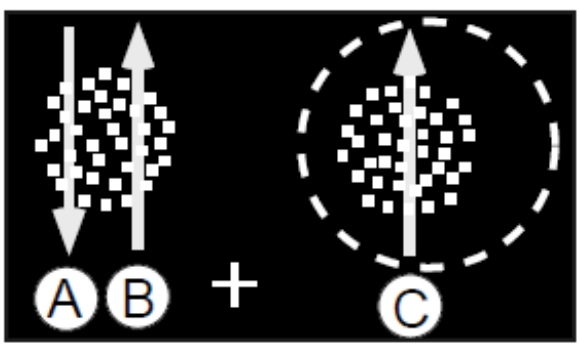

b

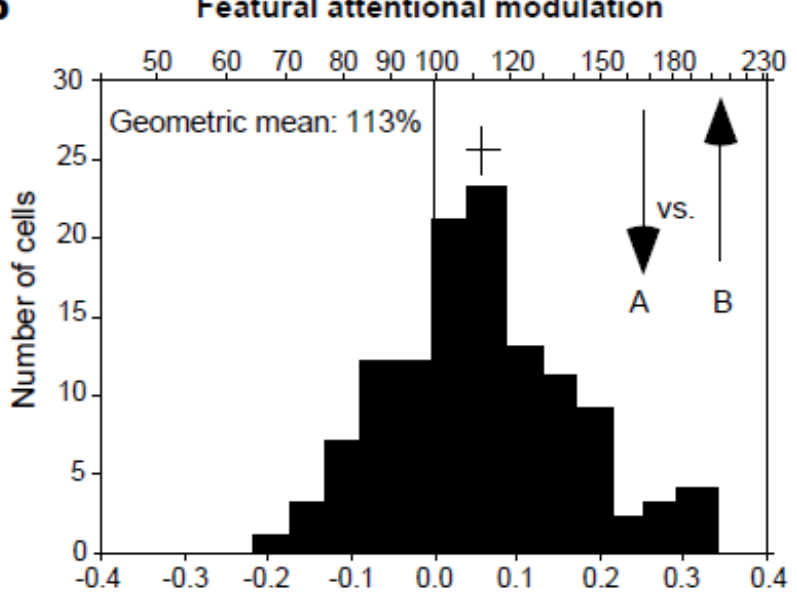

Figure 3: Effect of feature-based attention on the neuronal firing rate in area MT tuning curve. A: Experimental design: two random dot patterns (RDP) were presented inside the receptive field (indicated with the dashed circle) and outside the receptive filed. The RDP, which was inside the receptive filed, was moving at the preferred direction; and the other RDP had either the preferred or the null direction. B: distribution of feature-based attentional modulation was computed by comparing neuronal firing rate when attention was at the preferred $(B)$ versus anti-preferred $(A)$ direction outside the receptive field. The histogram showed $13 \%$ modulation (mean shift) by feature-based attention.

Treue, S. and J. C. Martinez-Trujillo (1999). "Feature-based attention influences motion processing gain in macaque visual cortex." N 399(6736): 575-579.

Reprinted by permission from Nature.

Furthermore, feature-based attention has been studied in several neuroimaging researches (Saenz et al., 2002; Serences and Boynton, 2007; Stoppel et al., 2011). It was shown that feature-based attention increases the hemodynamic responses to the ipsilateral distracter stimulus sharing the attended feature (Saenz et al., 2002). This modulation could even spread to un-stimulated neurons (Serences and Boynton, 2007). Moreover, it was reported that the magnitude of such a modulation depends on the similarity between the attended feature and presented stimulus (Stoppel et al., 2011). In summary, the converging electrophysiological, neuroimaging as well as psychophysics studies concluded that feature-based attention is a global mechanism that enhances the representation of the attended feature within the entire visual field. In chapter two, we examined the effect of feature-based attention on hemodynamic responses to 
the spiral motion patterns in addition to investigating its influence on the voxelbased tunings to the spiral motions.

A

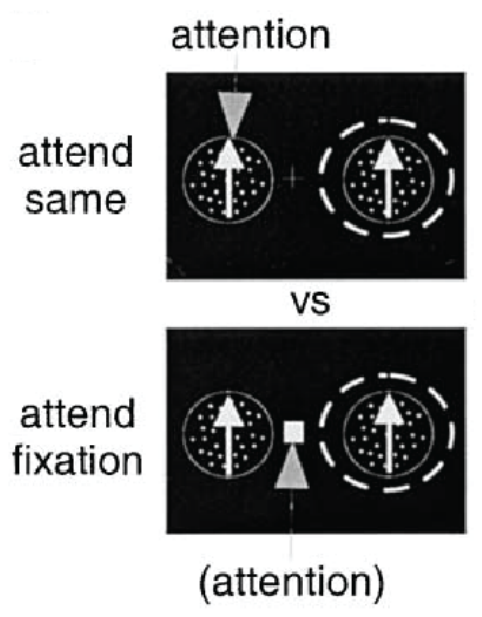

B

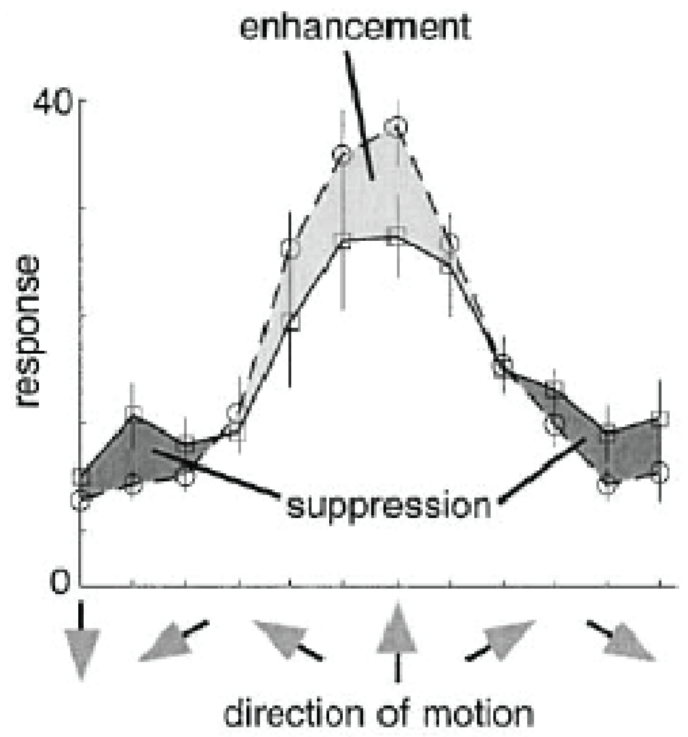

Figure 4: Effect of feature-based attention on the population responses in area MT tuning curve. A: Experimental design: two random dot patterns (RDP) with the same direction were presented to the receptive field (indicated with the dashed circle) and outside the receptive filed. The RDPs had one of the twelve sampled directions. B: directional tuning cureves. The upper tuning curve was determined when attention was to the same direction outside the receptive field (marked attend-same), and the lower tuning curve was extracted when attention was to the fixation point (marked attend-fixation). Feature-based attention enhances the selectivity of the population tuning curves.

Martinez-Trujillo, J. C. and S. Treue (2004). "Feature-based attention increases the selectivity of population responses in primate visual cortex." CB 14: 744-751.

Reprinted by permission from Cell. 


\section{Bibliography}

Albright, T.D., 1984. Direction and orientation selectivity of neurons in visual area MT of the macaque. Journal of Neurophysiology 52, 1106-1130.

Albright, T.D., Desimone, R., 1987. Local precision of visuotopic organization in the middle temporal area (MT) of the macaque. Experimental Brain Research 65, 582-592.

Awh, E., Pashler, H., 2000. Evidence for split attentional foci. Journal of Experimental Psychology: Human Perception and Performance 26, 834-846.

Becker, H.G., Haarmeier, T., Tatagiba, M., Gharabaghi, A., 2013. Electrical stimulation of the human homolog of the medial superior temporal area induces visual motion blindness. The Journal of neuroscience : the official journal of the Society for Neuroscience 33, 18288-18297.

Bichot, N.P., Cave, K.R., Pashler, H., 1999. Visual selection mediated by location: featurebased selection of noncontiguous locations. Percept Psychophys 61, 403-423.

Broadbent, D.E., 1958. Perception and Communication. Pergamon, London.

Cook, E.P., Maunsell, J.H.R., 2002. Attentional modulation of behavioral performance and neuronal responses in middle temporal and ventral intraparietal areas of macaque monkey. Journal of Neuroscience 22, 1994-2004

de Jong, B.M., Shipp, S., Skidmore, B., Frackowiak, R.S.J., Zeki, S., 1994. The cerebralactivity related to the visual-perception of forward motion in-depth. Brain 117, 1039-1054.

Desimone, R., Ungerleider, L.G., 1986. Multiple visual areas in the caudal superior temporal sulcus of the macaque. Journal of Comparative Neurology 248, 164-189.

Dubner, R., Zeki, S.M., 1971. Response properties and receptive fields of cells in an anatomically defined region of the superior temporal sulcus in the monkey. Brain Research 35, 528-532.

Duffy, C.J., Wurtz, R.H., 1991a. Sensitivity of MST neurons to optic flow stimuli. I. A continuum of response selectivity to large-field stimuli. Journal of Neurophysiology 65, $1329-1345$.

Duffy, C.J., Wurtz, R.H., 1991b. Sensitivity of MST neurons to optic flow stimuli. II. Mechanisms of response selectivity revealed by small-field stimuli. Journal of Neurophysiology 65, 1346-1359.

Duffy, C.J., Wurtz, R.H., 1995. Response of monkey MST neurons to optic flow stimuli with shifted centers of motion. Journal of Neuroscience 15, 5192-5208.

Duffy, C.J., Wurtz, R.H., 1997. Medial superior temporal area neurons respond to speed patterns in optic flow. Journal of Neuroscience 17, 2839-2851.

Eriksen, C.W., Yeh, Y., 1985. Allocation of attention in the visual field. Journal of Experimental Psychology: Human Perception and Performance 11, 582-597.

Freeman, T.C.A., Harris, M.G., 1992. Human sensitivity to expanding and rotating motion: Effects of complementary masking and directional structure. Vision Research 32, 81-87.

Fries, P., Reynolds, J.H., Rorie, A.E., Desimone, R., 2001. Modulation of oscillatory neuronal synchronization by selective visual attention. Science 291, 1560-1563.

Graziano, M.S.A., Andersen, R.A., Snowden, R.J., 1994. Tuning of MST neurons to spiral motions. Journal of Neuroscience 14, 54-67.

Hubel, D.H., Wiesel, T.N., 1968. Receptive fields and functional architecture of the monkey striate cortex. Journal of Physiology 195, 215-243. 
Huk, A.C., Dougherty, R.F., Heeger, D.J., 2002. Retinotopy and functional subdivision of human areas MT and MST. Journal of Neuroscience 22, 7195-7205.

Jans, B., Peters, J.C., De Weerd, P., 2010. Visual spatial attention to multiple locations at once: the jury is still out. Psychological Review 117, 637-684.

Kahnemann, D., 1973. Attention and Effort, 1st edition ed. N.J: Prentice Hall.

Kamitani, Y., Tong, F., 2005. Decoding the visual and subjective contents of the human brain. Nature Neuroscience 8, 679-685.

Kamitani, Y., Tong, F., 2006. Decoding seen and attended motion directions from activity in the human visual cortex. Current Biology 16, 1096-1102.

Kastner, S., De Weerd, P., Desimone, R., Ungerleider, L.G., 1998. Mechanisms of directed attention in the human extrastriate cortex as revealed by functional MRI. Science 282, 108-111.

Kastner, S., Pinsk, M.A., De Weerd, P., Desimone, R., Ungerleider, L.G., 1999. Increased activity in human visual cortex during directed attention in the absence of visual stimulation. Neuron 22, 751-761.

Kolster, H., Mandeville, J.B., Arsenault, J.T., Ekstrom, L.B., Wald, L.L., Vanduffel, W., 2009. Visual field map clusters in macaque extrastriate visual cortex. Journal of Neuroscience 29, 7031-7039.

Kolster, H., Peeters, R., Orban, G.A., 2010. The retinotopic organization of the human middle temporal area MT/V5 and its cortical neighbors. Journal of Neuroscience 30, 98019820.

Komatsu, H., Wurtz, R.H., 1988a. Relation of cortical areas MT and MST to pursuit eye movements I. Localization and visual properties of neurons. Journal of Neurophysiology 60, 580-603.

Komatsu, H., Wurtz, R.H., 1988b. Relation of cortical areas MT and MST to pursuit eye movements. III. Interaction with full-field visual stimulation. Journal of Neurophysiology $60,621-644$.

Lagae, L., Maes, H., Raiguel, S., Ziao, D.K., Orban, G.A., 1994. Responses of macaque STS neurons to optic flow components: A comparison of area MT and MST. Journal of Neurophysiology 71, 1597-1626.

Liu, T., Hou, Y., 2011. Global feature-based attention to orientation. Journal of Vision 11.

Liu, T., Mance, I., 2011. Constant spread of feature-based attention across the visual field. Vision Research 51, 26-33.

Luck, S.J., Chelazzi, L., Hillyard, S.A., Desimone, R., 1997. Neural mechanisms of spatial selective attention in areas V1, V2, and V4 of macaque visual cortex. Journal of Neurophysiology 77, 24-42.

Luck, S.J., Hillyard, S.A., Mouloua, M., Hawkins, H.L., 1996. Mechanisms of visual-spatial attention: resource allocation or uncertainty reduction ? Journal of Experimental Psychology: Human Perception and Performance 22, 725-737.

Martinez-Trujillo, J.C., Treue, S., 2004. Feature-based attention increases the selectivity of population responses in primate visual cortex. Current Biology 14, 744-751.

Maunsell, J.H.R., Newsome, W.T., 1987. Visual processing in monkey extrastriate cortex. Annual Review of Neuroscience 10, 363-401.

Maunsell, J.H.R., Van Essen, D.C., 1983a. The connections of the middle temporal visual area (MT) and their relationship to a cortical hierarchy in the macaque monkey. Journal of Neuroscience 3, 2563-2586. 
Maunsell, J.H.R., Van Essen, D.C., 1983b. Functional properties of neurons in the middle temporal visual area (MT) of the macaque monkey. I. Selectivity for stimulus direction, speed, and orientation. Journal of Neurophysiology 49, 1127-1147.

McAdams, C.J., Maunsell, J.H.R., 1999. Effects of attention on orientation-tuning functions of single neurons in Macaque cortical area V4. Journal of Neuroscience 19, 431441.

Meese, T.S., Anderson, S.J., 2002. Spiral mechanisms are required to account for summation of complex motion components. Vision Research 42, 1073-1080.

Moran, J., Desimone, R., 1985. Selective attention gates visual processing in the extrastriate cortex. Science 229, 782-784.

Morrone, M.C., Tosetti, M., Montanaro, D., Florentinii, A., Cioni, G., Burr, D.C., 2000. A cortical area that responds specifically to optic flow, revealed by fMRI Nature Neuroscience 3, 1322-1328.

Newsome, W.T., Paré, E.B., 1988. A selective impairment of motion perception following lesions of the middle temporal visual area (MT). Journal of Neuroscience 8, 2201-2211.

O'Connor, D.H., Fukui, M.M., Pinsk, M.A., Kastner, S., 2002. Attention modulates responses in the human lateral geniculate nucleus. Nature Neuroscience 5, 1203-1209.

O'Craven, K.M., Savoy, R.L., 1995. Attentional modulation of activation in human MT shown with functional magnetic resonance imaging (fMRI), Association for the Research in Ophthalmology (USA), Annual Meeting, p. S856.

Peuskens, H., Sunaert, S., Dupont, P., Van Hecke, P., Orban, G.A., 2001. Human brain regions involved in heading estimation. The Journal of neuroscience : the official journal of the Society for Neuroscience 21, 2451-2461.

Posner, M.I., 1980. Orienting of attention. Quarterly Journal of Experimental Psychology 32, 3-25.

Posner, M.I., Snyder, C.R.R., Davidson, B.J., 1980. Attention and the detection of signals. JEP-General 109, 160-174.

Saenz, M., Buracas, G.T., Boynton, G.M., 2002. Global effects of feature-based attention in human visual cortex. Nature Neuroscience 5, 631-632.

Saenz, M., Buracas, G.T., Boynton, G.M., 2003. Global feature-based attention for motion and color. Vision Research 43, 629-637.

Saproo, S., Serences, J.T., 2010. Spatial attention improves the quality of population codes in human visual cortex. Journal of Neurophysiology 104, 885-895.

Serences, J.T., Boynton, G.M., 2007. Feature-based attentional modulations in the absence of direct visual stimulation. Neuron 55, 301-312.

Serences, J.T., Saproo, S., Scolari, M., Ho, T., Muftuler, L.T., 2009. Estimating the influence of attention on population codes in human visual cortex using voxel-based tuning functions. Neuroimage 44, 223-231.

Shih, S.I., Sperling, G., 1996. Is there feature-based attentional selection in visual search? Journal of Experimental Psychology: Human Perception and Performance 22, 758-779.

Smith, A.T., Singh, K.D., Greenlee, M.W., 2000. Attentional suppression of activity in the human visual cortex. NeuroReport 11, 271-277.

Somers, D.C., Dale, A.M., Seiffert, A.E., Tootell, R.B.H., 1999. Functional MRI reveals spatially specific attentional modulation in human primary visual cortex. Proceedings of the National Academy of Sciences 96, 1663-1668.

Steiner, V., Blake, R., Rose, D., 1994. Interocular transfer of expansion, rotation, and translation motion aftereffects. Perception 23, 1197-1202. 
Stoppel, C.M., Boehler, C.N., Strumpf, H., Heinze, H.J., Noesselt, T., Hopf, J.M., Schoenfeld, M.A., 2011. Feature-based attention modulates direction-selective hemodynamic activity within human MT. Human Brain Mapping 32, 2183-2192.

Takeuchi, T., 1997. Visual search of expansion and contraction. Vision Research 37, 2083-2090.

Tanaka, K., Saito, H.-A., 1989. Analysis of motion of the visual field by direction, expansion/contraction, and rotation cells clustered in the dorsal part of the medial superior temporal area of the macaque monkey. Journal of Neurophysiology 62, 626-641.

Tootell, R.B., Mendola, J.D., Hadjikhani, N.K., Liu, A.K., Dale, A.M., 1998. The representation of the ipsilateral visual field in human cerebral cortex. Proceedings of the National Academy of Sciences of the United States of America 95, 818-824.

Tootell, R.B., Taylor, J.B., 1995. Anatomical evidence for MT and additional cortical visual areas in humans. Cerebral cortex (New York, N.Y. : 1991) 5, 39-55.

Treue, S., 2001. Neural correlates of attention in primate visual cortex. Trends in Neurosciences 24, 295-300.

(Treue, S., Martinez-Trujillo, J.C., 1999. Feature-based attention influences motion processing gain in macaque visual cortex. Nature 399, 575-579.

Treue, S., Maunsell, J.H.R., 1996. Attentional modulation of visual motion processing in cortical areas MT and MST. Nature 382, 539-541.

Ungerleider, L.G., Desimone, R., 1986. Projections to the superior temporal sulcus from the central and peripheral field representations of V1 and V2. Journal of Comparative Neurology 248, 147-163.

Van Essen, D.C., Maunsell, J.H.R., 1983. Hierarchical organization and functional streams in the visual cortex. Trends in Neurosciences, 370-375.

Yeshurun, Y., Carrasco, M., 1998. Attention improves or impairs visual performance by enhancing spatial resolution. Nature 396, 72-75.

Zeki, S., 1991. Cerebral akinetopsia (visual motion blindness). A review. Brain 114 ( Pt 2), 811-824.

Zeki, S., Watson, J.D.G., Lueck, C.J., Friston, K.J., Kennard, C., Frackowiak, R.S.J., 1991. A direct demonstration of functional specialization in human visual cortex. Journal of Neuroscience 11, 641-649.

Zeki, S.M., 1974. Functional organization of a visual area in the posterior bank of the superior temporal sulcus of the rhesus monkey. Journal of Physiology 236, 549-573. 


\section{Original manuscripts}

This chapter contains the following manuscripts:

2.1 Attentional modulation of human BOLD responses to spiral motion patterns

Sepideh Fazeli, Carsten Schmidt-Samoa, Peter Dechent, Stefan Treue prepared for submission.

2.2 The influence of spatial attention on human direction discrimination thresholds for spiral motion stimuli

Sepideh Fazeli, Stefan Treue prepared for submission. 


\subsection{Attentional modulation of human BOLD responses to spiral motion patterns}

In this chapter, we examined the effects of spatial and feature-based attention on the blood-oxygen-level-dependent (BOLD) signals in the MT and MST subregions of the human MT-complex in response to spiral motion patterns using functional magnetic resonance imaging (fMRI). Spatial attention modulated the BOLD signal in both MT and MST. We observed stimulus-selective modulation of the BOLD signal in the spiral-selective voxels in MST. Similarly, we saw a suppressive MSTspecific feature-based modulation.

\section{Author's contribution}

S. Fazeli and S. Treue designed the experiment. S. Fazeli performed data collection and analysis. S. The manuscript was written by S. Fazeli and edited by C. Schmidt-Samoa, P. Dechent and S. Treue. All authors discussed the results and worked on the manuscript. 


\title{
Attentional modulation of human BOLD responses to spiral motion patterns
}

\author{
Sepideh Fazeli ${ }^{1}$, Carsten Schmidt-Samoa ${ }^{2}$, Peter Dechent ${ }^{2}$, Stefan Treue ${ }^{1,3}$ \\ ${ }^{1}$ Cognitive Neuroscience Laboratory, German Primate Center, Goettingen, Germany \\ ${ }^{2}$ Department of Cognitive Neurology, University Medicine Goettingen, Goettingen, Germany \\ ${ }^{2}$ Bernstein Center for Computational Neuroscience, Goettingen, Germany
}

\section{ABSTRACT}

Visual attention allocates sensory processing resources to relevant information at the expense of other inputs deemed less relevant. Previous studies have shown the effects of spatial and feature-based visual attention in human with linear motion stimuli. In this study, we investigated spatial and feature-based attentional modulation in response to so called "spiral motion" patterns in the middle temporal area (MT) and the medial superior temporal area (MST) in healthy human subjects using functional magnetic resonance imaging (fMRI). We observed modulation of the hemodynamic response peak by spatial attention in MT and MST. In a subpopulation of voxels in MT and MST, which showed tuning to spiral motion patterns, we observed that spatial attention modulates the gain of such voxelbased tuning properties in MST and affects their baseline in MT. Such a stimulusselective modulation in MST suggests spiral visual motion as a preferred feature for MST but not for MT neurons. Feature-based attention suppresses the peak of hemodynamic responses only in MST. Similarly, feature-based attention scales down the gain of the voxel-based tunings only in MST. This indicates an areaspecific feature-based modulation. In conclusion, MST specific feature-based attention in addition to the feature-dependent spatial attention in MST point to it as the area most contributing to spiral motion processing, in line with previous studies in macaque visual cortex. 


\section{GENERAL INTRODUCTION}

In a given task, visual attention allocates sensory processing resources to the behaviorally relevant information at the expense of less significant inputs (Carrasco and McElree, 2001; Eriksen and Yeh, 1985; Pestilli and Carrasco, 2005; Posner et al., 1980; Yeshurun and Carrasco, 1999). Neuronal and behavioral correlates of attention have been extensively studied over the last two decades. The following papers should serve as examples: (Cook and Maunsell, 2004; Eriksen and Yeh, 1985; Gandhi et al., 1999; Posner, 1980; Serences, 2011; Treue and Martinez-Trujillo, 1999; Treue and Maunsell, 1996; Verghese et al., 2012). The efforts of the majority of visual attention researchers have been concentrated on investigating spatial attention as well as feature-based attention. Directing attention to a specific part of the visual space defines spatial attention; whereas, attending to a specific feature of a stimulus such as its color, orientation or motion direction is known as feature-based attention. Attention is called covert, if attentional allocation is accomplished without eye movement.

Converging electrophysiological, neuroimaging as well as psychophysics studies on spatial attention have shown that directing the spatial focus of attention to a target site increases the activity of neurons responding to it in the contralateral visual cortex (Buracas and Boynton, 2007; Gandhi et al., 1999; Murray and Wojciulik, 2004; Somers et al., 1999). It also increases the dynamic range of neuronal responses to the target's features such as direction of motion by scaling up their tuning functions (McAdams and Maunsell, 1999; Saproo and Serences, 2010; Serences et al., 2009; Treue and Maunsell, 1996). In addition, attention improves behavioral accuracy and speeds up responses to attended target (Carrasco and McElree, 2001; Desimone and Duncan, 1995; Eriksen and Yeh, 1985; Posner et al., 1980) with the cost of impaired performance at unattended locations (Desimone and Duncan, 1995; Pestilli and Carrasco, 2005).

In contrast, feature-based attention acts across the visual field as a global mechanism, which is supported by monkey electrophysiology studies (MartinezTrujillo and Treue, 2004; Maunsell and Treue, 2006; Treue and Martinez-Trujillo, 1999) as well as human neuroimaging findings (Saenz et al., 2002, 2003; Serences and Boynton, 2007). This means that feature-based attention is independent of the spatial focus of attention, and modulates the neuronal activity based on the attended feature. There are two primary models for neuronal 
mechanism of feature-based attention, known as "feature-matching" model (Motter, 1994) and "feature-similarity gain" model (Martinez-Trujillo and Treue, 2004; Treue and Martinez-Trujillo, 1999). According to the feature-matching model, the neuronal modulation reaches its maximum if the presented feature to the cell matches the attended feature. In other words, it is independent of the target's feature as well as of its similarity to the cell's preferred feature. Whereas, the feature-similarity model declares that feature-based attentional modulation depends on the similarity between the target's feature and the preferred feature of the cell. The feature-similarity gain model gets its main support from monkey electrophysiological data (Martinez-Trujillo and Treue, 2004; Treue and MartinezTrujillo, 1999). In addition, there are some studies in humans pointing toward the same conclusion (Liu et al., 2007; Saenz et al., 2002; Stoppel et al., 2011). As an example, in a functional magnetic resonance imaging (fMRI) study by (Stoppel et al., 2011), linear motion dot patterns were used with three levels of coherency. In each trial one direction was cued, which had the same or opposite direction to the probing stimulus, subjects were then asked to detect a fast movement in the stimulus. The highest modulation of the human MT hemodynamic activity was observed when the stimulus had the same direction as the cued direction in the $100 \%$ coherency. The lowest effect was seen when the stimulus contained the opposite direction of the cue. The inverse trend of the effect was observed using $70 \%$ coherency (i.e. lowest level of coherency). The Blood-oxygenation-level dependent (BOLD) signal in $\mathrm{fMRI}$ voxels are thought to reflect underlying neuronal activity which is mostly caused by active neurons in the inspected voxels (Sheinberg and Logothetis, 2001). Therefore, in this particular experiment, the hemodynamic activity was mostly related to the activity of neurons preferring the presented direction in highest coherency. In lowest coherency, the BOLD signal was linked to the firing of neurons preferring other directions. Thus, Stoppel et. al. interpreted their findings as a support for the "feature-similarity gain" model, which is expecting the highest increase of hemodynamic activity by attending to the preferred direction at the $100 \%$ coherency and lowest effect by attending to the opposite direction, while predicting the inverse effects at the low coherency condition.

Many human neuroimaging studies in the field of attention investigated the effects of attention on visual motion processing. The importance of visual motion to 
survival and the relative feasibility of using neuroimaging techniques to investigate visual areas specialized in visual motion processing explain such a trend (Kamitani and Tong, 2006; O'Craven et al., 1997; O'Craven and Savoy, 1995; Serences and Boynton, 2007; Somers et al., 1999). Optic flow is a pattern of visual motion, which results from relative movement between the observer and the scene. Spiral motion patterns are a class of optic flow patterns uniquely parameterized by the direction between their local speed and their radii (Graziano et al., 1994). Such characteristics of spiral motion patterns allow studying tuning properties of neurons in the visual cortex in response to them. It is widely accepted that visual areas located along the dorsal stream of the primate visual cortex are involved in motion processing in a hierarchical fashion (Maunsell and Newsome, 1987; Van Essen and Maunsell, 1983). Middle temporal (MT)-complex (termed also as hMT or $\mathrm{MT+}$ ) is a part of human dorsal stream, which is a specialized motion processing cluster. It contains the human homologues of the monkey's MT and the medial superior temporal (MST) areas (Kolster et al., 2010). Based on electrophysiological studies in macaque, MT is located below to MST in the visual motion processing hierarchy. Electrophysiology studies showed linear motion direction tuning properties in MT (Albright, 1984; Dubner and Zeki, 1971; Maunsell and Van Essen, 1983b). Higher in the visual hierarchy, neurons in MST get their input mostly from MT neurons (Maunsell and Van Essen, 1983a), show selectivity to optic flow patterns such as expansion and rotation (Duffy and Wurtz, 1991a, b) and show tuning properties to spiral motion patterns (Graziano et al., 1994). In the same line, human imaging studies provided evidence for linear motion direction selectivity (Kamitani and Tong, 2006; Zeki et al., 1991) as well as selectivity for optic flow patterns (de Jong et al., 1994; Smith et al., 2006; Wall et al., 2008) within human MT-complex. Although there are indications for selective responses to the cardinal directions in spiral motion space (i.e. expansion and rotation) within human MT-complex (Morrone et al., 2000; Wall et al., 2008), there is still a lack of direct evidence for spiral motion tuning in human visual cortex. In this chapter, we examined the existance of voxel-based tuning properties to spiral stimuli as well as how they are affected by spatial and feature-based attention within human MTcomplex.

We first localized MT and MST subregions of MT-complex using a standard localizer paradigm, based on the ipsilateral activation of MT and MST to a 
peripheral stimulus (Huk et al., 2002). It is widely known that the neuronal receptive field size increases in higher areas along the visual processing hierarchy (Desimone and Ungerleider 1986, Albright and Desimone 1987). A typical MT neuron has a relatively small receptive field rarely extended into the ipsilateral field, for example see: (Dubner and Zeki, 1971). In contrast, the size of a typical MST receptive field is big enough to extend into the ipsilateral field, for example see: (Desimone and Ungerleider, 1986). Such estimations of receptive field sizes of MT and MST neurons was obtained using electrophysiology in monkeys (Albright and Desimone, 1987; Desimone and Ungerleider, 1986; Duffy and Wurtz, 1991a; Komatsu and Wurtz, 1988; Tanaka and Saito, 1989), and are supported by human imaging studies (Huk et al., 2002; Kolster et al., 2010; Smith et al., 2006). Thus, a peripheral stimulus might activate MT in the contralateral hemisphere while activating MST in both contralateral and ipsilateral hemispheres, and thereby be used as a discriminative method to segregate MT and MST. Then, we aimed to answer whether spatial attention has an effect on the hemodynamic responses to spiral motion patterns in MT and MST subdivisions of human MT-complex. To that end, we conducted two experimensts. In the first experiment, we used a rapid event-related design and deconvolusion analysis (Dale and Buckner, 1997; Rosen et al., 1998) to determine the attentional modulation of the hemodynamic responses to the spiral motion stimuli. In the second experiment, we examined voxel-based tuning properties to spiral motion patterns as well as the influence of spatial attention on them in MT and MST. The concept of voxel-based tuning is constructed based on two assumptions. First, there should be a slight bias of population tuning preferences in each voxel to a particular feature, known as its preferred stimulus. Second, there should be a sensitivity of the vasculature in MT/MST to anisotropic distribution of feature-selective neurons (Kamitani and Tong, 2005; Serences and Saproo, 2010; Serences et al., 2009). Based on these assumptions, voxel's preferred stimulus can cause a bias in its responses, which could be detected in the BOLD signal. Thus, in the second experiment we measured the BOLD responses to twelve spiral directions for each voxel in order to extract its voxel-based tuning profile to spiral motion patterns. Then, we selected tuned voxels based on a novel criterion proposed in this study. We assessed the effects of spatial attention on the voxel-based tuning profiles using Monte Carlo cross-validation (Cao et al., 2007). In addition, we proposed a 
classifier, which read out the spiral direction presented to the subject by inferring the response of the tuned voxels in MT and MST. We then investigated the effects of attention on the performance of our classifier using Monte Carlo crossvalidation.

We also studied the general effects of feature-based attention on the hemodynamic responses to the expanding spiral patterns in the third experiment. Similar to the first experiment where spatial attention was investigated, we used rapid event related design and deconvolution analysis to estimate the hemodynamic responses in two conditions of attending to the same and opposite direction of the probe stimulus. In the fourth experiment, after selecting tuned voxels to spiral motion patterns based on our proposed inclusion criterion, we examined the influence of feature-based attention on the determined voxel-based tuning properties in MT and MST subdivisions of human MT-complex. Extracting the voxel-based tuning properties allows us to not only investigate the sensory encoding of complex motion patterns in the human MT-complex, but to examine modulatory effects of feature-based attention on such tuning properties to determine which model ("feature-similarity gain" or "feature-matching") fits our data best. In addition, similar to what was done in the second experiment, we assessed the effects of feature-based attention on the performance of our classifier, using Monte Carlo cross-validation.

\section{METHODS}

\section{General method}

\section{Participants}

Fourteen healthy volunteers with normal, corrected or acceptable vision (eight females), including one of the authors (SF) took part in this study. All participants (except SF) were naive to the aim of the study and gave their informed consent. Ethics committee of the psychology department of University Goettingen approved the study.

\section{Data acquisition}

FMR imaging was performed using a 3-Tesla Magnetom TIM TRIO scanner (Siemens Healthcare, Erlangen, Germany) with a twelve-channel head coil. Highresolution 3D Turbo FLASH anatomical images with the technical parameters of 
repetition time $(T R)=2250 \mathrm{~ms}$, echo time $(T E)=3.35 \mathrm{~ms}$, inversion time $=900 \mathrm{~ms}$, flip angle $9^{\circ}$ and voxel-size $=1 \times 1 \times 1 \mathrm{~mm}^{3}$ were acquired for each subject at the beginning of each session. The functional data was recorded using a gradientecho echo-planar imaging (EPI) sequence in 22 slices of $2 \mathrm{~mm}$ thickness per volume. The location and orientation of the slices were subjectively optimized to cover the medial temporal lobe and the primary visual cortex. The technical parameters for the functional scans were TE $=36 \mathrm{~ms}, \mathrm{TR}=2000 \mathrm{~ms}$, flip angle $70^{\circ}$, field-of-view $=192 \times 256 \mathrm{~mm}^{2}$, voxel size $=2 \times 2 \times 2 \mathrm{~mm}^{3}$ with interleaved acquisition order.

\section{Eye tracking}

Subjects were required and monitored to fixate exclusively a central fixation point, i.e. those runs where subjects had systematic eye movements (more than $10 \%$ of the trails) were dropped out of the analysis. Eye position was sampled at $60 \mathrm{~Hz}$ using a View Point Eye Tracker PC-60 (Arrington Research, Inc., Scottsdale, USA), a fiber optic MR-compatible eye tracker system.

\section{Stimuli}

The visual stimuli were pre-recorded as video files (avi format) in Psycho Toolbox, MATLAB (version R2011b, The MathWorks, Inc., Natick, Massachusetts, United States) and then presented using the stimulation software Presentation (Version 9.00, Neurobehavioral Systems, Albany, CA, USA) through LCD goggles with a resolution of $800 \times 600$ pixels creating a visual field of $32 \times 24$ deg $^{2}$ (Resonance Technology, Northridge, CA, USA).

The visual stimuli consisted of high contrast, bright moving dot patterns displayed on a dark background. The dot density was 9 dots $/ \mathrm{deg}^{2}$ and each dot was a square of $0.0064 \mathrm{deg}^{2}$.

The coherently moving spiral dot patterns (spiral stimuli) consisted of dots moving coherently along spiral trajectories based on the following equations:

$$
d r / d t=v \cos \phi, \quad d \theta / d t=\frac{v}{r} \sin \phi
$$

Where $r$ and $\theta$ are polar coordinates of each random dot and $d r / d t$ and $d \theta / d t$ are its radial and angular velocities respectively. Spiral parameters of $v$ and $\phi$ are speed and direction of the spiral motion. The local speed of every random dot is determined based on its distance from the center as $v=\alpha r$ where alpha is the proportionality constant factor termed as speed gradient. For a given stimulus, all 
dots move at the same angle relative to the radii, where, $\phi=0^{\circ}$ is pure expansion $(\mathrm{EXP}), \phi=90^{\circ}$ is pure clockwise rotation (CW), $\phi=180^{\circ}$ is pure contraction (CON) and $\phi=270^{\circ}$ is pure counterclockwise rotation (CCW). The spiral space is illustrated in Sup.Fig.1.

Spiral noise stimulus (randomly moving spiral dot patterns) and stationary dot patterns were used for the localizer experiment in addition to the spiral stimuli. In spiral noise stimulus, dots are moving incoherently with random direction but with the same gradient speed used in the spiral motion patterns.

In all experiments the speed gradient of spiral stimulus was $\alpha=3.2$. The average local speed of the probing stimulus was $8 \mathrm{deg} / \mathrm{sec}$.

\section{Data analysis}

The recorded data were analyzed using Brain Voyager QX 2.4 (Brain Innovation, Inc., Maastricht, The Netherlands). The preprocessing of functional data in each run consisted of slice time correction, 3D motion correction to compensate head motion and temporal high pass filtering to remove typical low frequency signal drift (2 cycles/run). Then the preprocessed functional data were co-registered to the anatomical scan, and transformed into the standard space of Talairach and Tournoux. The voxel size of resultant volume-time-course (VTC) was $3 \times 3 \times 3 \mathrm{~mm}^{3}$ for the localizer, the first experiment (spatial attention) as well as the third experiment (feature-based attention). Linear trend removal as well as spatial smoothing with a Gaussian kernel of $6 \mathrm{~mm}$ full width at half maximum was done on the normalized data in these experiments. The voxel size in second and fourth experiments was $2 \times 2 \times 2 \mathrm{~mm}^{3}$. With the smaller voxel size, we aimed to have higher spatial resolution to extract voxel-based tunings in MT and MST. We applied linear trend removal but not any spatial smoothing to the second and fourth experiments' VTCs to prevent integration of information from neighboring voxels.

\section{Localizer}

\section{Localizer experiment}

The localizer experiment consisted of two functional runs, each lasting 980 seconds. These runs were interspersed with a short break of about 5 minutes.

Each functional run started with the presentation of a circular ( $10^{\circ}$ diameter) spiral motion stimulus centered $10^{\circ}$ to the right of the central fixation point. The stimulus 
direction changed systematically every two seconds in a sequence of: $0^{\circ}, 60^{\circ}$, $120^{\circ}, 180^{\circ}, 240^{\circ}$ and $300^{\circ}$. After 12 second, an aperture filled with stationary dots replaced the spiral stimulus. The stationary stimulus had the same density, size and position as the spiral motion stimulus. The stationary dot pattern was displayed for another $12 \mathrm{sec}$, while the dots were randomly repositioned every 2 seconds to match the regular direction changes in the spiral motion stimulus. Following the stationary dots, the spiral noise stimulus was displayed for 12 seconds. It was then followed by another 12 seconds presentation of the stationary dot patterns.

This spiral stimulus - stationary dots - spiral noise- stationary dots cycle was repeated 10 times. After a rest period of $20 \mathrm{sec}$ (only blank screen) the same cycle of stimuli was repeated for another 10 times but the aperture was displayed at the left side of the fixation spot.

During the whole run, the subject was required to maintain the gaze on the central fixation spot while performing an attention task at the fixation point. The color of the fixation spot was changing randomly from white to one of the eight possible colors of: 'red: $R G B=[200,0,0]$ ', 'green: $R G B=[0,255,0]$ ', 'blue: $R G B=[0,0,200]$ ', 'gray: RGB=[100,100,100]', 'violet: $R G B=[255,0,255]$ ', 'yellow: $R G B=[255,255,0]$ ' and 'cyan: $R G B=[0,255,255]$ ' for $160 \mathrm{~ms}$. Two successive color changes were separated by $1500-8000 \mathrm{~ms}$. Subjects were tasked to press the response button as soon as they detect that the color of the fixation spot changed to red. The localizer paradigm is depicted in Fig.1.A.

\section{Localizer analysis}

To determine the regions of interest (ROIs) in each individual subject, the preprocessed data were fitted to a general linear model (GLM) to estimate the beta values. The regressors for the GLM were the convolution of the boxcar estimation of the neuronal responses to the stimulus in each condition with the hemodynamic impulse response function. We also segmented white matter and extracted its time course as an estimation of the physiological and measurement artifacts, e.g. heart rate, breathing, etc. The white matter time course was fed to the model as a confound predictor of the GLM analysis. 
A - localizer paradigm

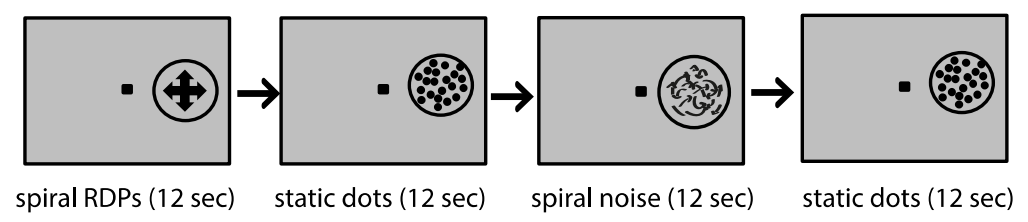

B - activity map of sample subject when stimuli were at right visual filed
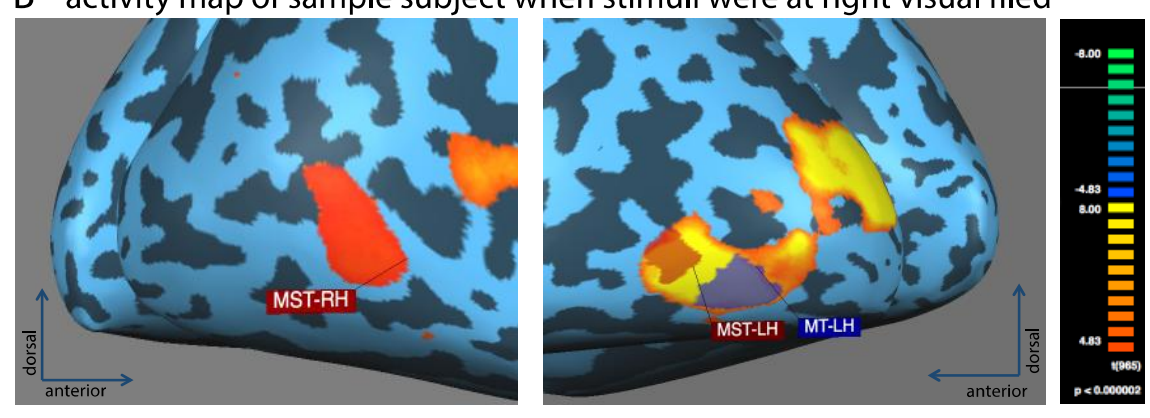

C - activity map of sample subject when stimuli were at left visual filed
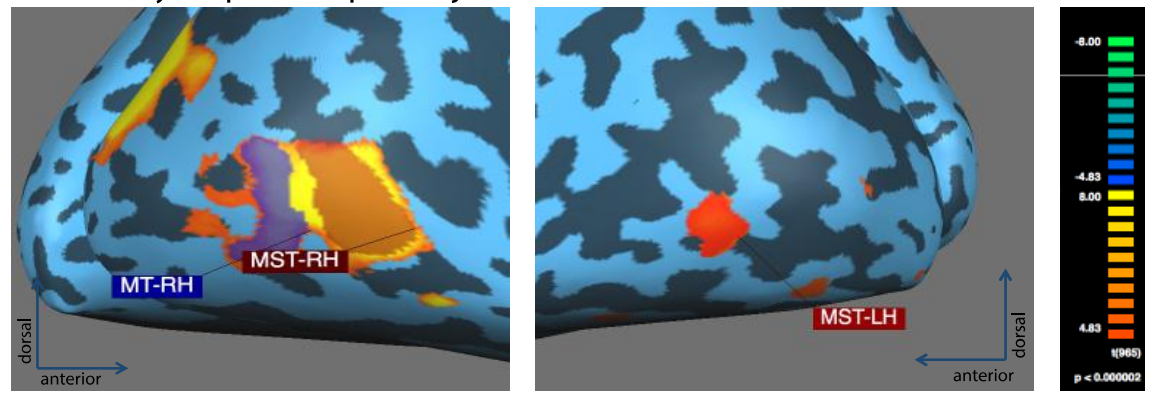

Fig. 1. Localizer experiment design and result of a sample subject

A: Localizer experiment design: The localizer experiment consisted of two functional runs, each with 20 trials. Each trial started by displaying a spiral stimulus ( $10^{\circ}$ diameter) at $10^{\circ}$ eccentricity in either the left (for first 10 trials in each run) or the right (for second 10 trials in each run) to the fixation point for $12 \mathrm{sec}$, followed by the stationary dot stimulus at the same location for another 12 sec. Then, the spiral noise stimulus was displayed for $12 \mathrm{sec}$ in the aperture, followed by another $12 \mathrm{sec}$ presentation of the stationary dots stimulus. The spiral stimulus changed its direction to every second. B: Sample subject beta map in the contrast of (right spiral stimulus - right stationary dots) was projected into his/her normalized inflated brain representation. MT-complex-LH and MST-RH are marked based on the contra and ipsilateral activity pattern in this contrast.

C: Sample subject beta map is illustrated in same way as the Fig.1.B but for the contrast of (left spiral stimulus - left stationary dots). Similar to the Fig.1.B, MT-complex-RH and MST-LH are marked. 
The whole MT-complex in each hemisphere was defined as the cluster, which was activated contralaterally at the contrast of the spiral stimulus versus stationary dots. MST was determined as the part of the MT-complex, which was activated both contra- and ipsilaterally; and finally MT was defined as the area which was activated only contralaterally and was located posterior to MST (Huk et al., 2002). To compensate for multiple comparison, the MT-complex activity maps were corrected using either a false discovery rate $(q(F D R)<0.05)$ or Bonferroni $(p($ Bonf $)<0.05)$ procedure depended on the activity map of each individual subjects in order to segregate MT-complex as an isolated cluster. MST maps were not corrected and the p-value threshold was set either to 0.01 or 0.05 to optimize signal to noise ratio.

For better visualization, the estimated beta map for each contrast was projected onto an inflated representation of each subject's anatomical data. To create the inflated brain, the high-resolution anatomical data was first normalized to the space of Talairach and Tournoux, corrected for the inhomogeneity, and after gray matter segmentation, the cortical mesh was created for each hemisphere, and inflated afterwards. All these steps were done using Brain Voyager V.2.4.

\section{Spatial Attention}

\section{Participants}

Twelve healthy volunteers (eight females, age 23-35 years), including one of the authors (SF) took part in the localizer session. AS, BK, CT, KK, LK, MS, PL, and SN participated in the first experiment and CT, JL, KF, KK, LK, PL, SF, SN and TJ in the second experiment.

\section{Spatial attention experiments}

To investigate the effect of spatial attention, two experiments were performed. The first experiment was designed to study the general effect of attention on the hemodynamic responses. Spiral motion voxel-based tuning properties and any attentional modulation of such properties were specifically investigated in the second experiment.

\section{First experiment}

The first experiment consisted of four functional runs each contained 60 trials. These runs were separated with a short break of about 5 minutes. Every trial started with the appearance of a fixation spot, followed by a central cue after $1000 \mathrm{~ms}$. The cue was a triangle pointing randomly to either left or right, indicating 
the location of the upcoming target stimulus. $500 \mathrm{~ms}$ after the appearance of the cue two expanding spiral stimuli $\left(10^{\circ}\right.$ diameter) were displayed for $3 \mathrm{sec}$, centered at $10^{\circ}$ eccentricity to the right and the left. Each stimulus underwent 0-3 speed changes. Subjects had to report the number of speed changes of the target stimulus during the following inter-trial interval. The experimental paradigm is depicted in Fig.2. The duration of these inter-trial intervals were randomly jittered between $1 \mathrm{sec}$ and $16 \mathrm{sec}$.

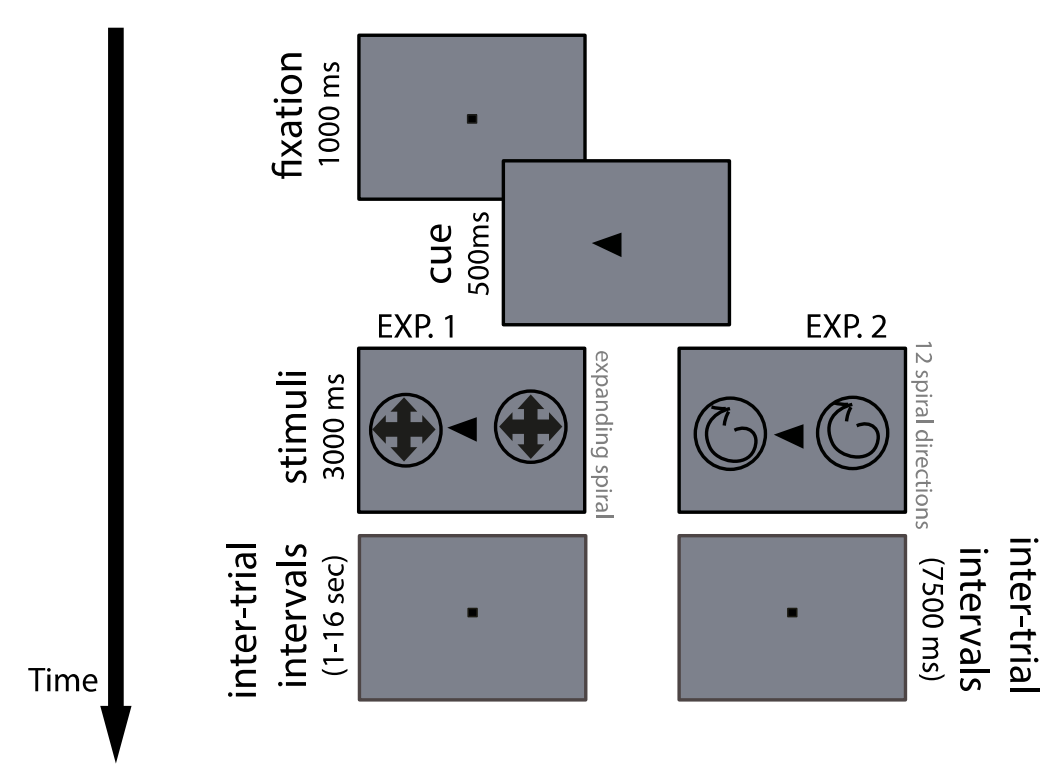

\section{Fig.2. Spatial attention experiment design}

Every trial started by the appearance of a fixation spot. After $1000 \mathrm{~ms}$ a central triangle (the cue) was displayed pointing to either the left or right, indicating the upcoming target location. After $500 \mathrm{~ms}$ two identical circular ( $5^{\circ}$ radius) spiral random dot patterns were displayed for $3 \mathrm{sec}$. The spiral stimuli were centered at $10^{\circ}$ eccentricity to the right and the left of the fixation spot. Each stimulus contained 0-3 speed changes occurring with the same probability. In the first experiment, in each trial the spiral stimuli contained expanding spirals, whereas, in the second experiment the spiral stimuli had one of the twelve equally spaced spiral directions. Subjects had to report the number of speed changes at the target stimulus during the coming inter-trial interval. The inter-trial interval was randomized between 1-16 seconds for the first experiment, and was fixed to $7500 \mathrm{~ms}$ in the second experiment.

\section{Second experiment}

The trial design in the second experiment was the same as the first experiment with two differences. Both spiral stimuli had one direction out of the twelve possible directions of: $0^{\circ}, 30^{\circ}, 60^{\circ}, 90^{\circ}, 120^{\circ}, 150^{\circ}, 180^{\circ}, 210^{\circ}, 240^{\circ}, 270^{\circ}, 300^{\circ}$, 
$330^{\circ}$. Moreover, the inter-trials intervals were fixed to $7500 \mathrm{~ms}$. All the other parameters were the same as in the first experiment.

\section{Analysis}

\section{Attention Modulation}

To investigate the effects of spatial attention we conducted two experiments. In the first experiment, we determined the effect of attention on the hemodynamic responses to expanding $\left(0^{\circ}\right)$ spirals using a uni-variate analysis. In the second experimnet, we investigated the spatial attentional modulation to different spiral directions using voxel-based tuning analysis. We also examined attentional effect on the classifier performance based on the tuned voxels response profile.

\section{The effect of attention on the hemodynamic response}

Deconvolution analysis of the BOLD signal in the rapid event-related design was used to examine attentional modulation of the BOLD response to spiral stimulus. For the deconvolution analysis, we used a general linear model (GLM) to estimate the hemodynamic response at all sampled time points, in each attention condition (Dale and Buckner, 1997). This estimation of the hemodynamic response is based on the assumption of linear contribution of the preceding trials on the BOLD signal. This criterion was met as the inter-stimulus intervals in our experiment were longer than 2.5 seconds (Dale and Buckner, 1997). Moreover, the estimation of the hemodynamic responses would only converge successfully if the inter-trial intervals are properly jittered and the conditions are balanced. This condition was also met in our design by jittering the time separating two successive events between 5.5-19.5 sec, while attention conditions were randomized and balanced. Indeed, jittering of the inter-trial intervals guaranties a random mixture of effects of preceding trials (conditions) at different time points of the BOLD signal. Therefore, the GLM design matrix is not singular and its inverse matrix can be solved in order to separate the effects of preceding trials from the BOLD signal, and ultimately estimate the hemodynamic response in each condition (Dale and Buckner, 1997). Furthermore, the high temporal resolution of the jittering (1s) than the TR (2s) provided us with different mixtures of the preceding trials in the temporal resolution of one second, which consequently allowed us to increase the temporal resolution of the estimated hemodynamic response to a temporal resolution of one second. Hence, the hemodynamic response for each attentional condition was estimated using the deconvolution analysis. The deconvolution analyses was done in Brain 
Voyager QX 2.4. The estimated hemodynamic responses were then averaged across all voxels in each $\mathrm{ROI}$ for every individual subject. We then tested the main hemisphere effects as well as the interaction between hemisphere and attention using full factorial repeated measured ANOVA across all subjects. The rmANOVA had three levels of time (estimated time points of the hemodynamic response, hemisphere and attention condition). If there was no systematic difference between the activation patterns across hemispheres, we averaged the estimated responses across the left and right ROIs for each subject. Finally, we computed the attentional modulation of the hemodynamic activity (averaged across the hemispheres) over its peak, i.e. from 4 to 8 second after the spiral stimuli onset in MT and MST. Attentional modulation index was computed as follows:

attentional index $=\frac{\text { attend contra }- \text { attendipsi }}{\text { attend contra }+ \text { attendipsi }}$

The Wilcoxon sign ranked test was applied for the statistical comparisons between attentional conditions. All the statistical analysis was done in MATLAB (version R2011b, The MathWorks, Inc., Natick, Massachusetts, United States).

\section{Attention modulation of population tuning profiles}

In the second experiment, we aimed to extract voxel-based tuning in each voxel of MT and MST.

First, the volume time course from each voxel was normalized on a run-by-run basis using z-transformation to remove the effect of different scanning runs. Then, for each voxel the BOLD response to each event was estimated as the difference between the maximum value of the BOLD signal over the time window of 4 to 12 second after the stimuli onset, which covered the BOLD signal peak, and its minimum over the last two seconds before the stimulus onset represented the baseline. The estimated responses to all events in all four runs determined the BOLD response time course in each voxel. We normalized the response time course of each voxel using softmax normalization explained in the following equation:

$$
\mathrm{y}=\operatorname{softmax}(\mathrm{x})=\exp (\mathrm{x}) / \operatorname{sum}(\exp (\mathrm{x}))
$$


Then, the response profile (tuning function) in each voxel was computed as the function that is mapping the average of its responses to each presented spiral direction independent of the attentional condition.

In order to select the tuned voxels and examine their tuning in each ROI, we introduced an inclusion criterion. The tuning function of each voxel was transferred into the twelve vectors corresponding to the twelve spiral directions. Each vector had the angle of $\theta$ as its corresponding spiral direction and the amplitude of $r$ as the mean response of the voxel to the direction of $\theta$. Then, we computed vectorial sum of these twelve vectors in each voxel. The vectorial sum operator gave two values: the amplitude and the direction. For a tuned voxel, this amplitude should be significantly bigger than the absolute mean of the tuning profile vectors as described in the following equation:

$$
\left|\sum_{i=1}^{n} \vec{r}_{i}=\right|>\frac{\sum_{i=1}^{n}\left|\vec{r}_{i}\right|}{n}
$$

As an illustrative example, we simulated a Gaussian (tuned) response profile and a noisy response function. Then, we computed the amplitude of their vectorial sum as well as the absolute mean of their response profiles (depicted in Sup.Fig.2) to demonstrate the result of our suggested criterion in these two extreme cases.

We used bootstrapping to simulate the distribution of the suggested tuning criterion in each voxel. Then, we tested if the mean of the criterion distribution is bigger than zero using ttest at $5 \%$ statistical significant. All the voxels that satisfied the inclusion criterion were used for further tuning analysis. For the tuning analysis, we used Monte Carlo cross validation (Cao et al., 2007) with $75 \%$ of the events for the training and the remaining $25 \%$ for the test. In each iteration the preferred direction of each voxel was determined as the direction of its tuning function vectorial sum independent of the attentional conditions using the training data. Then the tuning functions of the voxels in test data set were aligned to their preferred direction in each attentional condition separately. Sup.Fig.3 illustrated the flowchart for computing the voxel-based tunings.

Moreover, we checked the soundness of our inclusion criterion in selecting tuned voxels by simulating noisy volume time courses with both normal distribution (mean $=0, s t d=1$ ) and Poisson distribution $(\lambda=1)$. Our inclusion criterion accepted only less than $0.05 \%$ of the noisy voxels as tuned voxels. We also used a 
permutation based testing as a sanity check for the inclusion criterion by randomly exchanging the labels of directions. Using such a permutation test, less than $5 \%$ of the voxels were selected based on our inclusion criterion.

If there is neural tuning to spiral patterns with anisotropic distribution within voxels, and the vasculature in MT/MST is sensitive to this anisotropic distribution, then we might see a systematic BOLD response pattern to different spiral stimuli, i.e. means the evoked response patterns are more similar for the close stimuli in spiral space. Thus, Gaussian function is a proper candidate to model such a tuning profile. To examine the voxel-based tunings properties for each individual subject,

a circular Gaussian function of $f(x)=a+b \times e^{c \times(\cos (x-d)-1)}$ was fitted to the tuning profiles across the accepted tuned voxels in each $\mathrm{ROI}$ and each attentional condition separately. Then, the baseline, the amplitude, the bandwidth and the center of the fitted Gaussians were determined. The modulation indices of these parameters were computed to investigate the effects of spatial attention on the voxel-based tuning properties.

All the analysis was done in MATLAB (version R2011b, The MathWorks, Inc., Natick, Massachusetts, United States).

\section{Decoding of the presented direction with and without attention}

Tuning to sensory features in the brain is thought to be essential for perception. Although classification techniques such as support vector machines provided evidence for the emergence of perception in the sensory areas such as primary visual cortex, they failed to justify the impact of neuronal tuning on the classifier performance directly (Kamitani and Tong, 2005, 2006).

To study the direct association between voxel-based tunings and the classifier performance, we implemented a linear decoder for each attentional condition. It used the responses of the tuned voxels as its input to decode the direction of the presented spiral. In its first level, all tuned voxels that passed our tuning criterion were divided into twelve groups based on their preferred direction. Each group (we termed it spiral group) represented one direction out of the twelve sampled spiral directions. In the second step, the response values to each event were averaged across the contributing voxels in each spiral group (for MT and MST separately). Therefore, there were twelve values for each event, corresponding to each spiral group. A weighted vectorial sum (where each group had a weight of Wi ) of these values was computed in the third level. The direction of the vectorial sum 
determined the presented spiral direction. Our proposed decoder was evaluated using Mote Carlo cross validation with $75 \%$ of the events for the training and the remaining $25 \%$ for the test. In each iteration, the tuned voxels were grouped based on their preferred direction determined by the training events. The weights of these groups (Wi) were determined using the least square optimizer to minimize the error of the model in decoding spirals using the training data set. Then the performance of the model was determined using the test events.

\section{Feature-based Attention}

\section{Participant}

Twelve healthy subjects (eight females, age 23-35), including one of the authors (SF) took part in the localizer session. BK, CT, LK, JL, MO, PL, SF, SN, TL and TJ participated in the third experiment and CT, JL, KF, LK, MS, MO, PL, SF, SN and $\mathrm{TJ}$ in the fourth experiment.

\section{Feature-based attention experiments}

Feature-based attention was studied in two experiments. The first experiment aimed at optimizing the investigation of the main effects of feature-based attention on the hemodynamic responses. The second experiment allowed us to assess the encoding of the spiral motion patterns reflected in the BOLD signal and their modulation by feature-based attention. Besides, we examined the decoding of spiral motion pattern using the fMRI time courses of MT and MST.

\section{Third experiment}

The third experiment consisted of four functional runs each with 60 trials. These runs were interspersed with short breaks of about 5 minutes. Subjects had to foveate a central fixation point. Every trial started by displaying a central fixation point for $1000 \mathrm{~ms}$. It followed by displaying a central cue (a triangle pointing to one of the screen quarters to indicate the upcoming target) for $500 \mathrm{~ms}$. Then three spiral moving stimuli, centered at either $\left[\left(10^{\circ}, 0^{\circ}\right),\left(-8^{\circ}, 4^{\circ}\right)\right.$ and $\left.\left(-8^{\circ},-4^{\circ}\right)\right]$ or $[(-$ $\left.10^{\circ}, 0^{\circ}\right),\left(8^{\circ}, 4^{\circ}\right)$ and $\left.\left(8^{\circ}, 4^{\circ}\right)\right]$ relative to the center of the screen, were displayed for $3 \mathrm{sec}$. These spiral stimuli had a diameter of $10^{\circ}, 9.6^{\circ}$ and $9.6^{\circ}$ respectively. The probe stimulus in this study was the horizontal midline centered stimulus, which contained expanding spiral. Therefore, the cue always pointed to one of the other two patches as the upcoming target. The upper non-probe stimulus contained expanding (same direction as probe stimulus) and the lower one contained contracting (opposite direction to the probe stimulus) spiral moving pattern. Each 
of the non-probe spiral stimuli underwent none, one, two, or three speed changes, each case having equal probability of occurrence. Subjects had to report the number of speed changes at the target stimulus during the following inter-trial interval. Inter trial intervals were jittered between $1 \mathrm{sec}$ and $16 \mathrm{sec}$. The experimental paradigm is depicted in Fig.3.

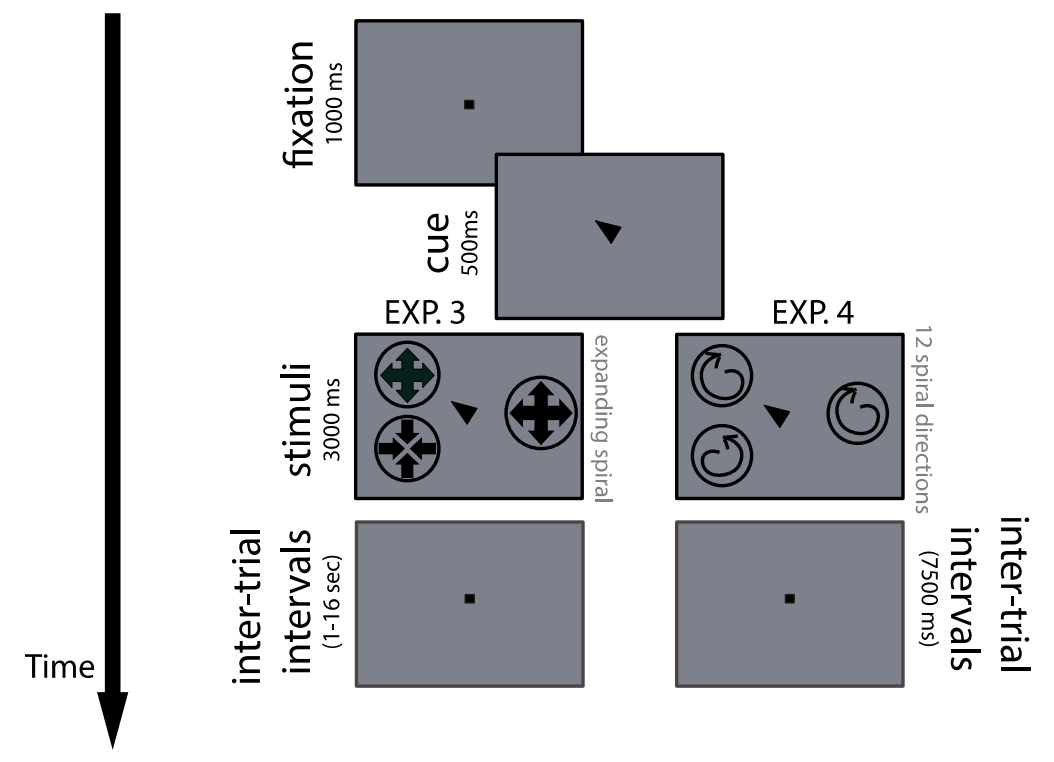

\section{Fig.3. Feature-based attention experiment design}

Every trial started by the appearance of a fixation spot for $1000 \mathrm{~ms}$. Then the central triangle cue was displayed for $500 \mathrm{~ms}$ pointing randomly to one of the visual quadrates, indicating the upcoming target location. Afterwards, three circular apertures filled with spiral random dot patterns (RDPs) were displayed for $3 \mathrm{sec}$. The three patches centered at either $[(10,0),(-8,4)$ and $(-8,-4)]$ or $[(-$ $10,0),(8,4)$ and $(8,-4)]$ coordinates with diameter of $10^{\circ}, 9.6^{\circ}$ and $9.6^{\circ}$ respectively, where $(0,0)$ is the coordinate of the fixation spot. The spiral patch centered on the horizontal midline is the probe stimulus. The upper non-probe stimulus contained spiral RDPs with the same direction as the probe stimulus and the lower one contained spiral RDPs with the opposite direction to the probe stimulus. Each of the non-probe stimuli contained 0-3 speed changes happening with the same probability. In the third experiment, the probe stimulus was an expanding spiral, whereas, in the fourth experiment the probe stimulus had one of the twelve equally spaced spiral directions in each trial. Subjects had to report the number of speed changes at the target stimulus during the next inter-trial interval. The inter-trial interval was randomized between 1-16 seconds for the third experiment, and was fixed to $7500 \mathrm{~ms}$ in the fourth experiment.

\section{Fourth experiment}

The trial design in this experiment was the same as the third experiment with two differences. The probe spiral stimulus had one direction out of the twelve possible 
directions of $0^{\circ}, 30^{\circ}, 60^{\circ}, 90^{\circ}, 120^{\circ}, 150^{\circ}, 180^{\circ}, 210^{\circ}, 240^{\circ}, 270^{\circ}, 300^{\circ}, 330^{\circ}$. The upper non-probe stimulus had the same spiral direction as the probe stimulus and the other one contained spiral stimulus with the opposite direction. Moreover, the inter-trials intervals were set fixed to $7500 \mathrm{~ms}$. All the other parameters were the same as the third experiment.

\section{Analysis}

\section{Feature-based attention modulation}

We studied the effects of feature-based attention in the third and the fourth experiments. There, we only used the contralateral voxels to probe stimulus for the analysis. Considering the design of the experiment, these voxels were in the ipsilateral hemisphere to the allocation of spatial attention.

In the third experiment, we aimed to use same analytical approach as the first spatial attention experiment. Feature-based attentional modulation index was computed as follows:

attention index $=\frac{\text { attend same }- \text { attend ops }}{\text { attend same }+ \text { attend ops }}$

In the fourth experiment, we normalized the volume time course of each voxel on a run-by-run basis using z-transformation same as the experiment two. Then, in each voxel we estimated the BOLD response to each event as the modulation of the maximum value of the BOLD signal over the time window of 4 to 12 second after the stimuli onset (corresponding to its peak) and its minimum over the last two seconds before the stimulus onset. Then, we appended together the estimated responses from the four runs and normalized the resultant time course using min-max normalization. The voxel-based tuning profiles were computed in a same way as the experiment two. We also selected the tuned voxels based on the same inclusion criterion mentioned before. A circular Gaussian function with the same equation was fitted to the voxel-based tunings in each attention condition for MT and MST. We determined the baseline, the amplitude, the bandwidth and the center of the fitted Gaussians, and computed the modulation indices for these fit parameters to examine the influence of feature-based attention on voxel-based tunings.

All the analysis was done in MATLAB (version R2011b, The MathWorks, Inc., Natick, Massachusetts, United States). 


\section{RESULT}

\section{Localizer}

MT and MST sub-regions of the human MT-complex were localized using the described localizer paradigm. Our localizer was designed based on the paradigm first proposed by (Huk et al., 2002). We modified their task by using twelve spiral directions rather than expanding spiral. We used the contrast of peripheral spiral stimulus versus stationary dot patterns to segregate MT-complex in the contralateral hemisphere and MST in the ipsilateral hemisphere. Highlighted by this contrast, MT was also defined as a cluster activated only in contralateral hemisphere. We also considered the ascending limb of inferior temporal sulcus (ITS) as an anatomical landmark for the approximate location of the human MTcomplex. Our localized MSTs were mostly anterior/dorsal to the ITS and the MTs in most cases were defined at its posterior/ventral adjacent region. In other words, MST was more anterior/dorsal to MT; i.e. $Y$ coordinates of MSTs were bigger than MTs (Wilcoxon sign ranked test, $\mathrm{p}<0.05$ ). Fig. $1 \mathrm{~B}-\mathrm{C}$ illustrated the activity maps of one sample subject for the contrast of "spiral stimulus versus stationary dots" projected onto the inflated brain. The identified MT and MST are also depicted. We were successful to segregate MT and MST in both hemispheres of twelve subjects out of fourteen using our localizer contrast of "spiral stimulus vs. stationary dots". In the other two participants, the localizer contrast could not elicit any ipsilateral activity in one hemisphere (for $\mathrm{BK}$ in right and for $\mathrm{JL}$ in left hemisphere). Therefore, we failed to identify MT/MST in that hemisphere for those two volunteers. In those two cases, we only used the data from the other hemisphere. The standard Talairach coordinates of the localized MTs and MSTs in the population of fourteen volunteers were summarized in Table 1.

Table 1- Averaged Talairach coordinates of the center of the MT and MST subregions of MTcomplex in the left and right hemispheres

\begin{tabular}{lcccccc}
\hline & \multicolumn{2}{c}{$\mathrm{LH}$} & \multicolumn{3}{c}{$\mathrm{RH}$} & $\mathrm{X}$ \\
Area & $\mathrm{X}$ & $\mathrm{Y}$ & $\mathrm{Z}$ & $\mathrm{X}$ & $\mathrm{Y}$ & $\mathrm{Z}$ \\
MT & $-43 \pm 1.2$ & $-77 \pm 1$. & $-6 \pm 1.9$ & $37 \pm 1.3$ & $-74 \pm 1$. & $-6 \pm 1.3$ \\
MST & $-44 \pm 1.2$ & $-69 \pm 1.5$ & $-7 \pm 1.1$ & $38 \pm 0.9$ & $-64 \pm 1.2$ & $-3 \pm 1.7$ \\
\hline mean \pm SE & & &
\end{tabular}


The identified locations of MT and MST segregated by our localizer were approximately at the same Talairach coordinates as what had already been shown by (de Jong et al., 1994; Morrone et al., 2000) for the human MT-complex; but at the inferior extreme of MT and MST sub-regions of MT complex reported by (Dukelow et al., 2001; Kolster et al., 2010; Smith et al., 2006). This inconsistency between the results could originate from the use of different type of stimuli (twelve directions of spiral random dot patterns (RDP) vs. expanding/contracting RDPs) or different eccentricity of the peripheral stimulus. The second reason seems more unlikely, as MST is known as an area with no clear retinotopic organization (Huk et al., 2002; Kolster et al., 2010).

Furthermore, as we did not have any retinotopic mapping to distinguish MT and MST, we might have some voxels assigned to the wrong cluster.

Given together, we would rather call the segregated regions by our localizer putative-MST (pMST) and putative-MT (pMT).

Moreover, we used the Talairach coordinates of MT and MST reported by (Kolster et al., 2010) (Sup.Table1) to recalculate both the spatial and feature-based attentional modulation in order to verify our attention results and prove the reliability of our extracted ROIs.

\section{Spatial attention}

We recorded the BOLD responses from 12 human subjects ( 8 females, age 23-35 years). In the two spatial attention experiments, subjects had to attend to one of two RDPs, placed to the right and left of the central fixation point while maintaining their gaze at the central fixation point.

\section{Spatial attentional modulation of the hemodynamic response}

We investigated the attentional modulation of the hemodynamic responses to the expanding spiral RDPs when attention was on the contralateral stimulus vs. the ipsilateral one. In this analysis, we estimated the hemodynamic response to the spiral stimulus using the deconvolution analysis in the rapid event-related design in PMT and PMST of each hemisphere separately. We first check for the hemisphere effect and did not observe any difference of the estimated hemodynamic response between left and right hemispheres, either in pMT (rmANOVA, hemisphere effect: $F=2.47, p=0.16$, interaction of hemisphere and attention: $F=0.81, p=0.63$ ) or in pMST (rm-ANOVA, hemisphere effect: $F=1.67$, 
$p=0.1$, hemisphere and attention interaction: $F=0.81, p=0.63)$. Then we averaged the estimated hemodynamic responses across both hemispheres for each individual subject. The averaged hemodynamic responses in the population of eight subjects are depicted in panels A and B of the Fig. 4 for pMT and pMST respectively. The plot shows the peak modulation of the hemodynamic response when attention was at the contralateral stimulus vs. the ipsilateral one. Then, we computed the attentional modulation indices as described in the methods. Panel $\mathrm{C}$ of the Fig.4 demonstrates the box plots of the attentional modulation indices in the population of eight subjects for pMT and pMST. There, we observed a $13 \%$ (median) modulation of the peak response in pMT and 10\% (median) modulation in PMST when attention was shifted from the ipsilateral visual field to the contralateral one ( $\mathrm{pMT} \& \mathrm{pMST}, \mathrm{p}=0.08$, Wilcoxon sign ranked test).

\section{Spatial attentional modulation of population voxel-based tunings}

We examined the voxel-based tunings to the spiral stimuli, and the effects of spatial attention on them in PMT and PMST sub-regions of human MT-complex. We aimed to see whether there was a systematic BOLD response pattern to spiral RDPs similar to neural tuning obtained using electrophysiology method by (Graziano et al., 1994), i.e. neighbor stimuli in spiral space evoke similar response patterns than more distant stimuli in the spiral space. Hence, we computed the response profile of each voxel to the presented twelve directions in PMT and pMST. Next, the tuned voxels were selected using our suggested inclusion criterion. About $39 \pm 1 \%$ of pMT and $33 \pm 8 \%$ of pMST voxels were selected as tuned voxels across nine subjects. For each tuned voxel, we computed the voxelbased tuning profile and aligned it to its preferred direction using Monte Carlo cross validation in both attention conditions separately. Then we averaged the extracted tuning profiles across the tuned voxels to reveal the population voxelbased tuning profile in PMT and PMST for each subject in each attention condition. Fig. 5, panels $A$ and $B$ show the voxel-based tunings averaged across nine subjects in each attention condition, for PMT and PMST respectively. It can be seen that spatial attention modulates the population voxel-based tuning properties. To check this effect quantitatively, we fitted a circular Gaussian function to the population voxel-based tuning of each subject, and computed the attentional modulation indices for fit parameters. Fig.5.C and Fig.5.D illustrate the attentional modulation indices for each fitted parameter across nine subjects in PMT and 
PMST respectively. We observed $90 \%$ additive modulation of the baseline of the fitted Gaussians ( $p=0.01$, Wilcoxon sign ranked test) in $p M T$ and $a+8 \%$ modulation (median) of the amplitude of the fitted Gaussians $(p=0.03$, Wilcoxon sign ranked test) in PMST with spatial attention. Spatial attention had no significant effect on the amplitude in pMT ( $p=0.5$, Wilcoxon sign ranked test), the baseline in pMST ( $p=0.25$, Wilcoxon sign ranked test), the bandwidth in both pMT and pMST (pMT: $p=0.16$ \& pMST: $p=0.2$, Wilcoxon sign ranked test) and the center of the Gaussians in both areas ( $\mathrm{pMT}: \mathrm{p}=0.91$ \& $\mathrm{pMST}$ : $\mathrm{p}=0.16$, Wilcoxon sign ranked test).

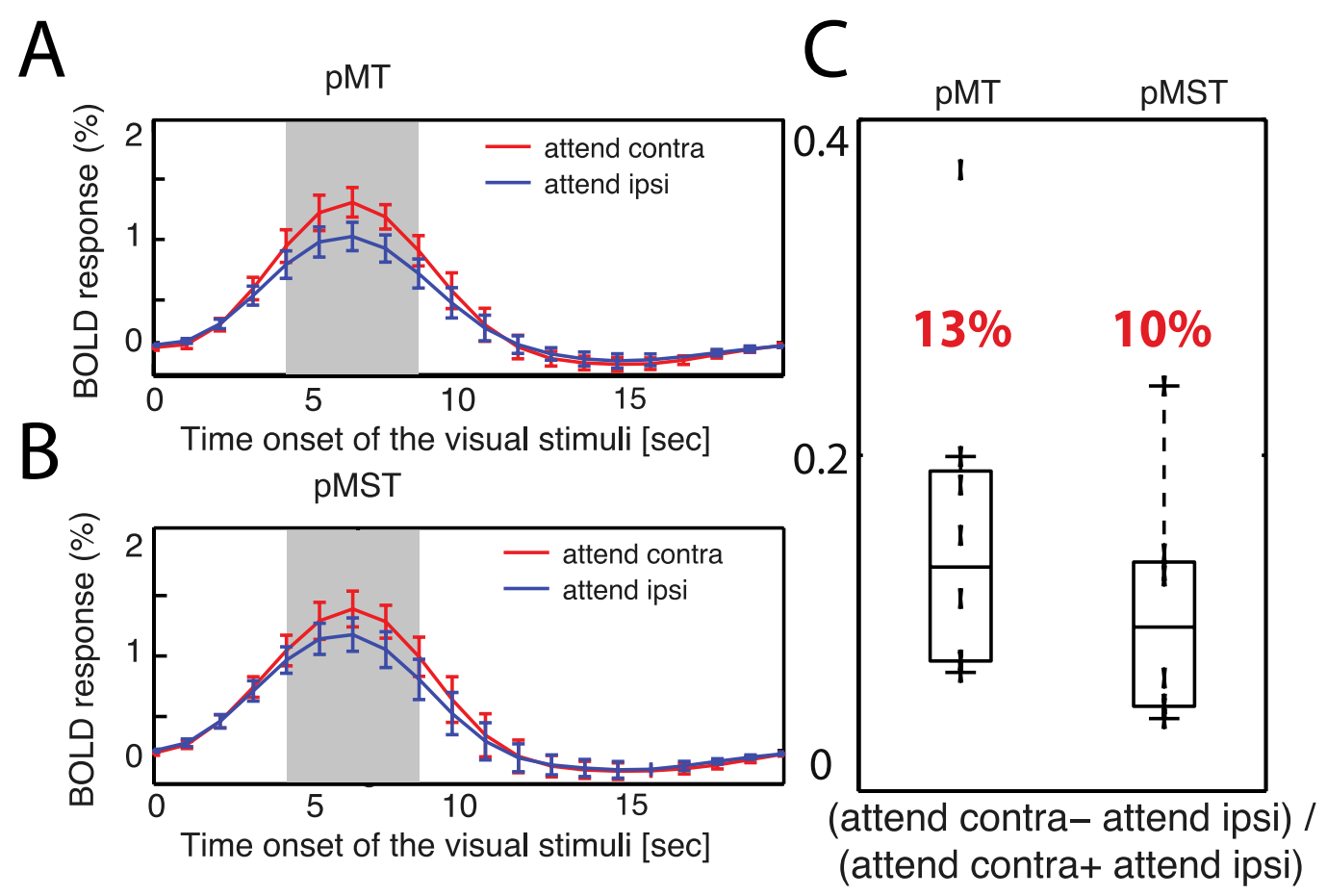

Fig.4. Spatial attentional modulation of the hemodynamic response peak to the expanding spirals

(A) The average of the hemodynamic responses to the expanding spiral across eight subjects depicts for two attentional conditions in PMT. The red curve corresponds to the attend-contra condition and the blue curve corresponds to the attend-ipsi condition. The error bars represent standard error of the population mean. (B) Averaged pMST hemodynamic response (plotted in a same way as in the Fig.4.A). (C) Spatial attentional modulation indices across eight subjects are plotted for pMT and pMST. Attention modulation indices had the median of $13 \%$ in pMT ( $p=0.008$ Wilcoxon sign ranked test) and $10 \%$ in pMST ( $\mathrm{p}=0.008$ Wilcoxon sign ranked test). 

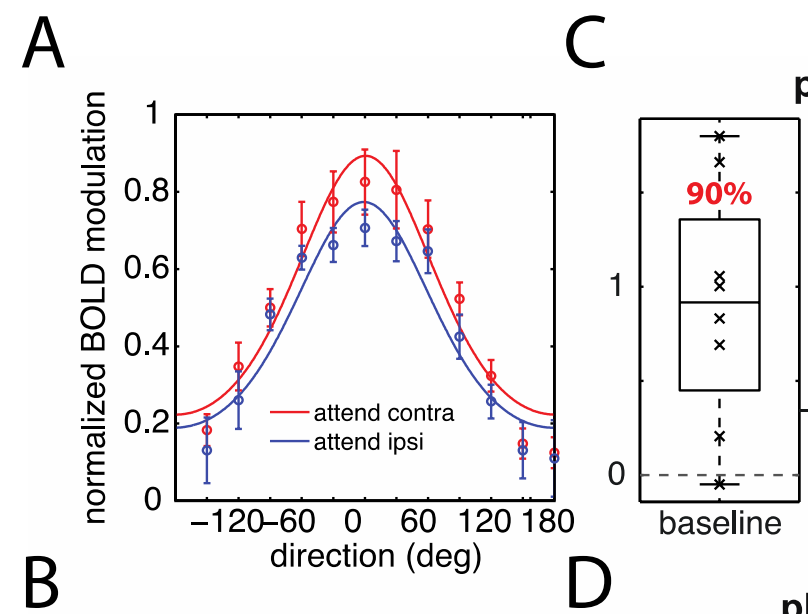

PMT
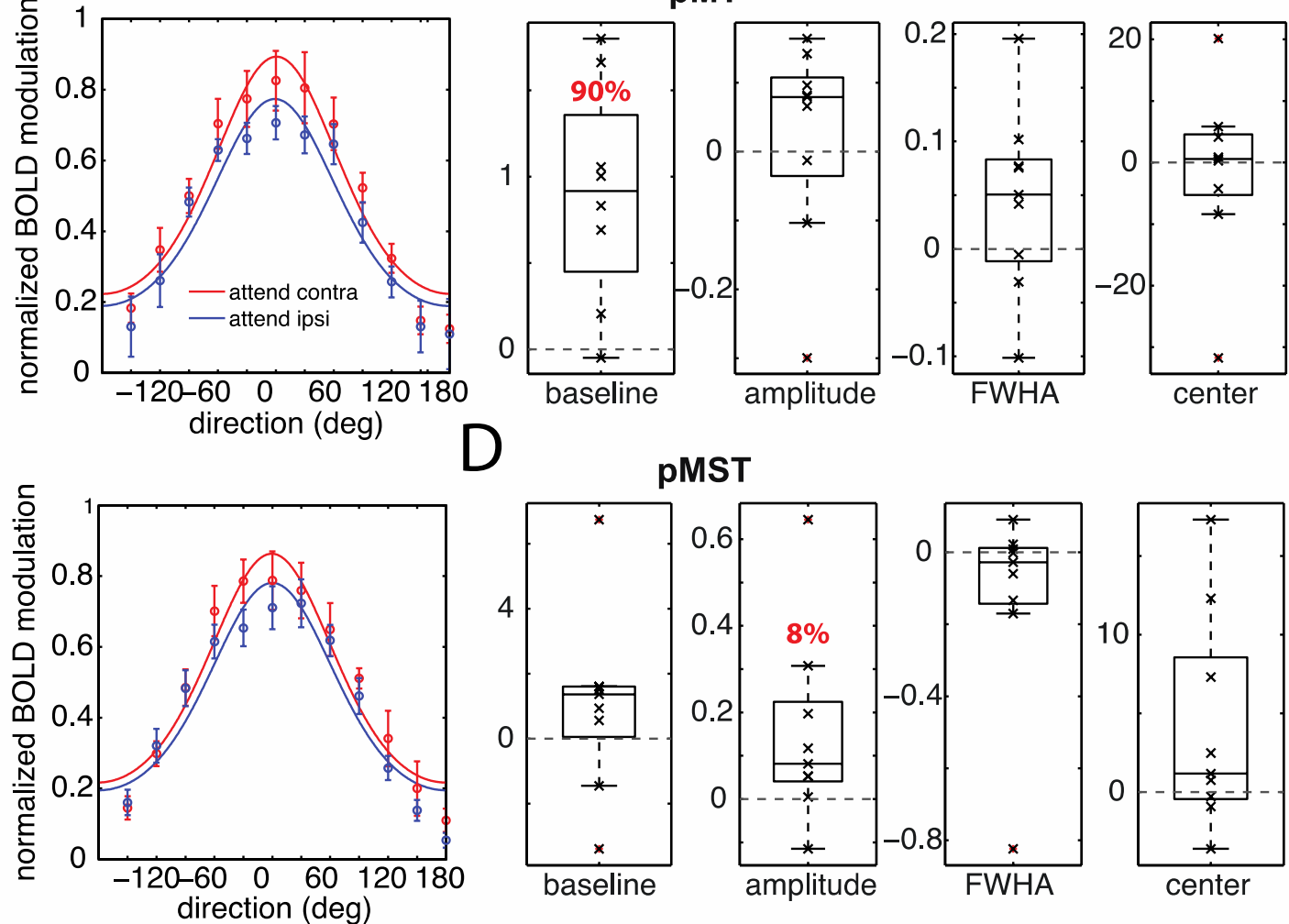

pMST
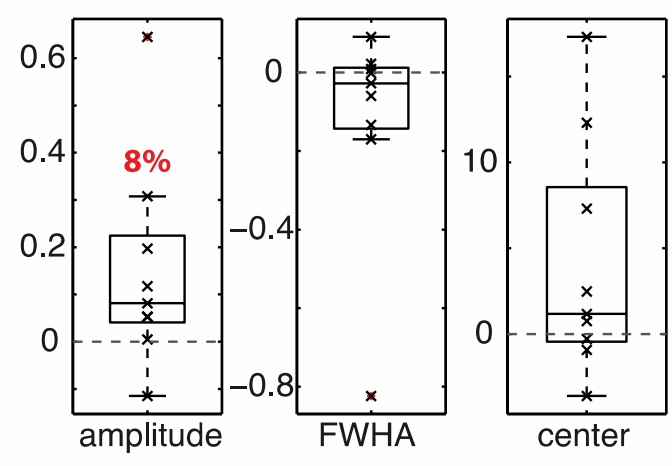

\section{Fig.5. Spatial attention modulation of the voxel-based tuning functions}

(A) Population voxel-based tunings: For each voxel the aligned voxel-based tuning was mapping the BOLD response to the difference between the presented direction and the preferred direction. The voxel-based tunings were max-min normalized and averaged across all tuned voxels to create the population voxel-based tuning in the PMT for each subject. The population voxel-based tuning averaged across the population of nine subjects in two attention conditions of 'attend-contra' and 'attend-ipsi' and plotted. Error bars represent the standard error of the population mean. (B) Average of the population voxel-based tuning functions are plotted for PMST in the same way as in panel A. (C) The resultant population voxel-based tuning for each subject was fitted to a circular Gaussian to determine the modulation of the voxel-based tuning fit parameters. The boxplots show the attention modulation indices ('attend contra' - 'attend ipsi')/ ('attend contra' + 'attend ipsi') for the parameters of the fitted Gaussians. There was a significant baseline modulation (median $90 \%$, $p=0.01$ Wilcoxon sign ranked test) in pMT. (D) The attention modulation indices of the fitting parameters are plotted for PMST as in the panel C. We saw a significant modulation of the Gaussian amplitude (median $8 \%, p=0.03$ Wilcoxon sign ranked test). 
A

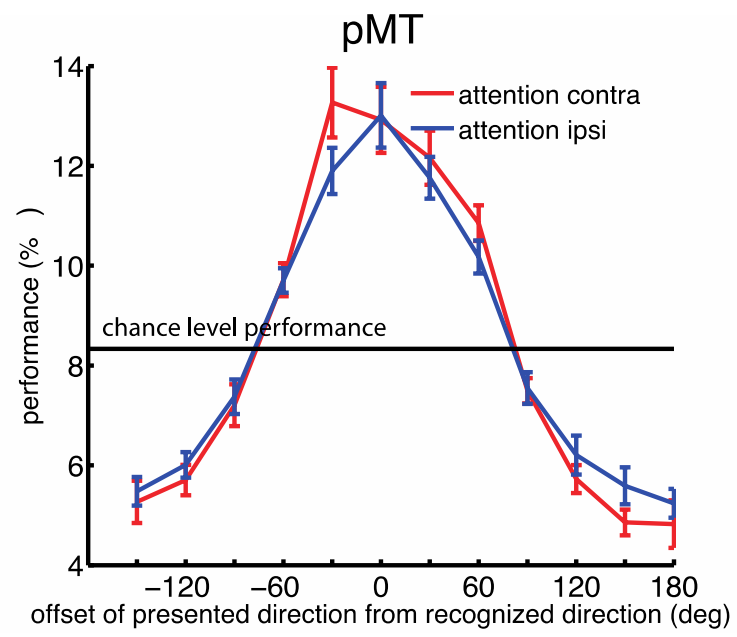

B

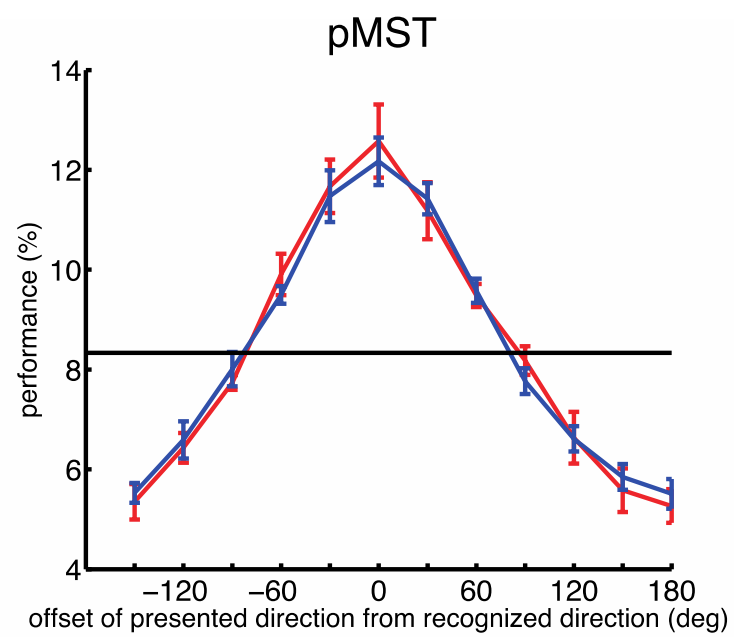

Fig.6. Decoding of the presented spiral with and without spatial attention

(A) The decoder performance using the data from pMT voxels is depicted as a function of the difference between the presented direction and the predicted direction. There were two decoders corresponding to the two attention conditions. For each decoder, first voxels were grouped into twelve groups based on their preferred direction. In each group, the mean of the BOLD responses to their corresponding spiral direction across all their contributing voxels was computed as its value. The weighted vectorial sum of the groups' values indicated the presented spiral direction. The decoder performance in two attention conditions of 'attend-contra' and 'attend-ipsi' was averaged across the population of eight subjects and plotted. Error bars represent the standard error of the population mean. There was no significant modulation of the decoder performance by attention. (D) The decoder performance is plotted as in panel A for area pMST. There was no significant modulation of the classifier performance by attention.

\section{Decoding the presented direction with and without spatial attention}

Neuronal tuning of spiral directions might contribute to the perception of optic flow patterns. Thus, we designed a decoder to decode the presented spiral direction based on the response of the tuned voxels in PMT and pMST. We also examined the influence of attention on the performance of our decoder by decoding the direction of the presented spiral stimulus with and without attention. The performance of the decoder was evaluated using Monte Carlo cross validation. Fig.6 depicts its performance by using tuned voxels in PMT and pMST. Our decoder successfully decoded the direction presented to it independent of attention better than chance level (which is 1/12) (Wilcoxon sign ranked test, $\mathrm{p}=0.01$ ). Our decoder demonstrated a promising enhancement of its performance with spatial attention (rmANOVA, main attention effect: $F=3.79, p=0.09$ ). Its performance improved from $11.9 \%$ to $13.2 \%$ (mean) in pMT (rmANOVA, $F=9.88$, 
$p=0.01$ ); but did not change in pMST. However, we observed no difference of decoder performance between pMT and pMST (rmANOVA, main area effect: $F=$ 3.33, $p=0.11$, area $x$ attention: $F=2.35, p=0.16$ ).

\section{Feature-based attention}

We recorded the fMRI data from 12 human subjects (8 females, age 23-35 years). During attention experiments subjects had to attend to one of the vertically aligned RDPs while maintaining their gaze at the central fixation point. The other unattended RDP at the opposite visual field is our probe stimulus. The upper stimulus contained RDPs with same direction as the probe one and the lower one contained RDPs moving in opposite direction to the probe stimulus. The localized voxels in contralateral hemisphere to the probe stimulus were examined as MT and MST ROls to determine feature-based attention modulation.

\section{Feature-based attentional modulation of the hemodynamic response}

We aimed to assess the feature-based attentional modulation of the hemodynamic responses to spiral motion patterns. Therefore, we compared the estimated hemodynamic responses to the probe stimulus, i.e. the expanding spiral stimulus when attention was directed to the same direction vs. the opposite direction.

We used deconvolution analysis of a rapid event-related design to estimate the hemodynamic responses to the expanding spiral stimulus in PMT and PMST in each hemisphere separately. We saw neither a significant main hemisphere effect (rmANOVA, MT: $F=0.1, p=0.75$; MST: $F=0.098, p=0.76$ ), nor an interaction of attention and hemisphere (rmANOVA, MT: $F=1.05, p=0.32$; MST: $F=0.19, p=0.66$ ). Then for each individual subject we averaged the estimated hemodynamic responses in their ROIs across both hemispheres. The averages of the estimated hemodynamic responses in the population of ten subjects are depicted in panels $\mathrm{A}$ and $B$ of the Fig.7 for PMT and PMST respectively. We can see a suppression of the peak response in PMST but not in pMT. Thus, we computed the attentional modulation of the hemodynamic responses over their peak in pMT and PMST. The box plots of the attentional modulation in PMT and PMST in the population of ten subjects is plotted in panels $\mathrm{C}$ of the Fig.7. There, we observed a $-3 \%$ (median) modulation of the peak of the estimated hemodynamic response in PMST when attention was directed from the opposite stimulus to the same within the ipsilateral 
visual field to the probe stimulus $(p=0.03$, Wilcoxon sign ranked test). No modulation of the hemodynamic activity occurred in pMT.
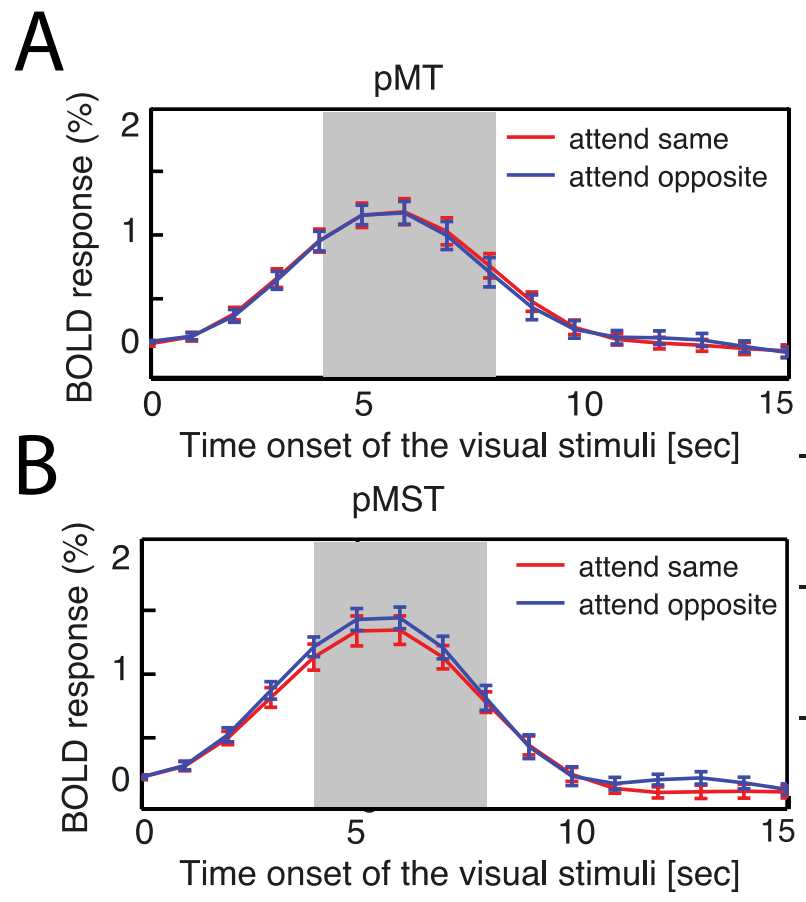

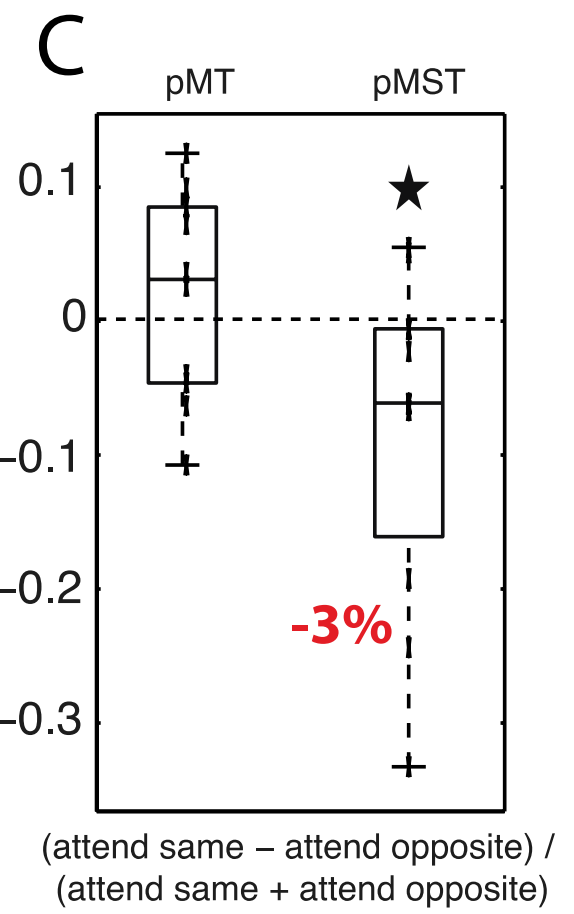

Fig.7. Feature based attentional modulation of the hemodynamic response peak to the expanding spirals

(A) Estimated hemodynamic responses to the expanding spiral were averaged across 10 subjects when attention is directed either to the same (red) direction or opposite (blue) direction within the ipsilateral visual field to the probe stimulus in area pMT. The error bars represent standard error of the population mean. (B) Hemodynamic responses averaged across the population of 10 subjects in pMST plotted in as same way as in the Fig.7.A. (C) Distribution of the feature based attention modulation indices in PMT and PMST are plotted. Feature-based attention modulated the hemodynamic response peak only in pMST (median: $-3 \%, p=0.03$ Wilcoxon signed rank test).

\section{Feature-based attentional modulation of population voxel-based tunings}

We examined voxel-based tunings and the effect of feature-based attention on them in PMT and PMST subregions of human MT complex.

As a first step, we only included tuned voxels based on our suggested inclusion criterion for the further tuning analysis. About $22 \pm 2 \%$ of pMT and $20 \pm 2 \%$ of PMST voxels were selected as tuned voxels. At this step, we had to exclude one subject (JL) because of the small number of the tuned voxels in his ROls. For this subject we had only the voxels form the right hemisphere as we failed to localize MT and MST in left hemisphere. 
The response profile of each voxel in the ROls at both attend-same as well as attend-opposite conditions were estimated using Monte Carlo cross validation and were aligned to their preferred directions. They were averaged across all the tuned voxels to extract the population voxel-based tuning in PMT and PMST for each subject. Fig.8, panels $A$ and $B$ show the population tuning profile averaged across nine subjects, in both attention same and opposite conditions, in PMT and pMST respectively.

Then we determined the parameters of the fitted Gaussians to the voxel-based tuning in each attentional condition. Feature-based attentional modulation indices of each fitted parameter across nine subjects are illustrated in Fig. 8.C and Fig. 8.D for PMT and pMST respectively.

We observed $-12 \%$ (median) modulation of the amplitude of the fitted Gaussians in PMST when attention was shifted from the opposite spiral direction to the same one within the ipsilateral visual field ( $p=0.03$, Wilcoxon sign ranked test). Featurebased attention had no significant effect on the baseline $(p=0.7$, Wilcoxon sign ranked test), bandwidth ( $p=0.5$, Wilcoxon sign ranked test) as well as the center ( $p=0.9$, Wilcoxon sign ranked test) of the Gaussians in pMST. Moreover, featurebased attention had no significant effect on none of the fitting parameters in PMT (Wilcoxon sign ranked test, baseline: $p=0.35$, amplitude: $p=0.5$, bandwidth: $p=0.2$ and center: $\mathrm{p}=1$ ).

\section{Decoding the presented direction with and without feature-based attention}

Similar to the second experiment, we examined the contribution of tuned voxels in PMT and PMST in decoding of spiral directions in addition to studying the influence of feature-based attention on our decoder performance. We decoded the direction of the presented spiral stimulus with and without feature-based attention using our proposed decoder. The performance of our decoder was evaluated using Monte Carlo cross validation. Fig.9.A-B depicts the performance of our proposed decoder based on the data extracted from the tuned voxels in PMT and PMST in attentionsame and attention opposite conditions. Our model successfully decoded the presented direction to it independent of the attended featured (Wilcoxon sign ranked test, $p=0.0001$ ). The decoder did not demonstrate any enhancement of its performance by attending to different features either in pMT (rmANOVA, $F=1.79$, $\mathrm{p}=0.22$ ) or in $\mathrm{pMST}$ (rmANOVA, $\mathrm{F}=0.68, \mathrm{p}=0.43$ ). Moreover, we observed no 
difference in the decoder performance between pMT and PMST (rmANOVA, main area effect: $F=1.54, p=0.25$; area $x$ attention: $F=0.38, p=0.55)$.

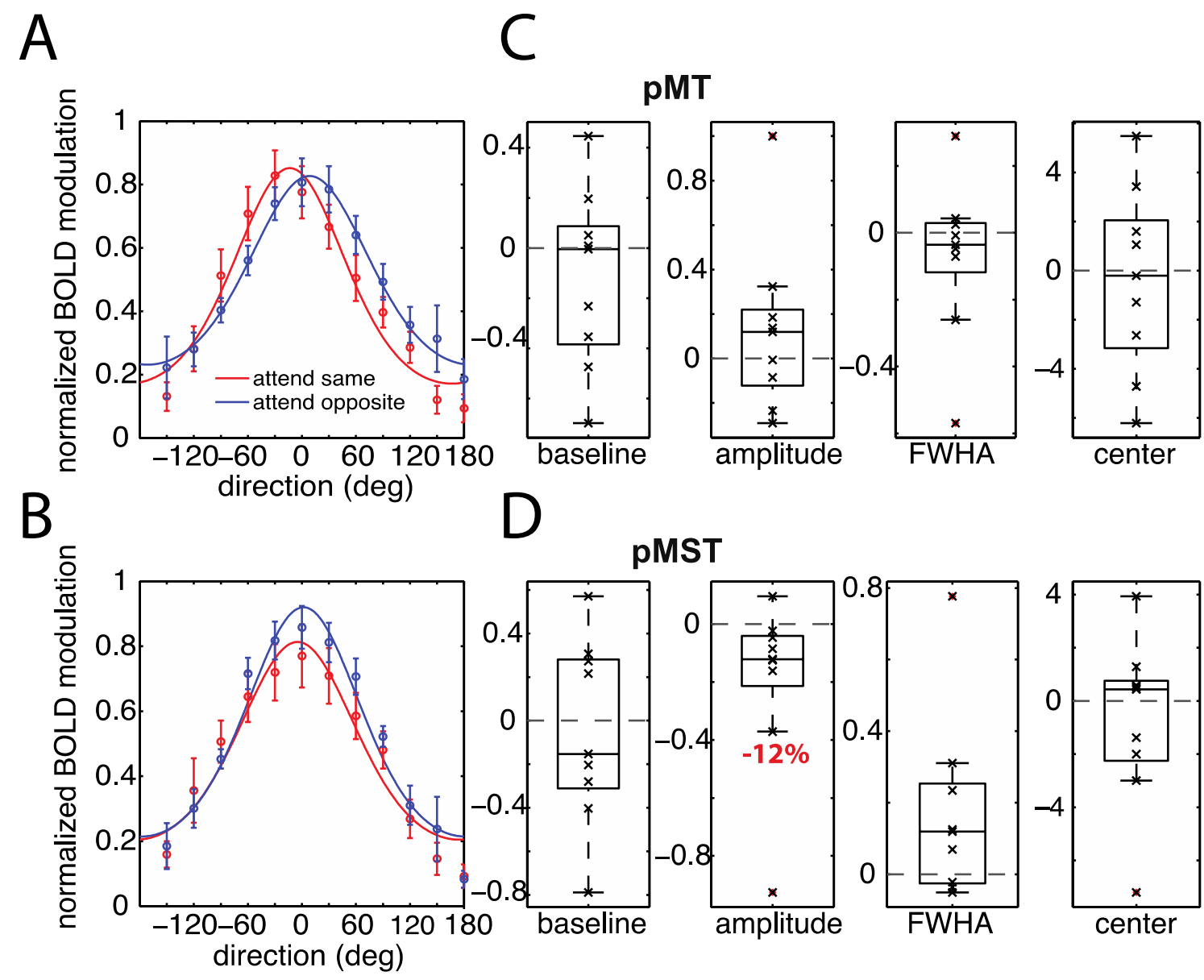

Fig.8. Feature based attentional modulation of the voxel-based tuning functions

(A) Population voxel-based tunings: For each voxel the aligned voxel-based tuning mapped the BOLD response to the difference between the presented direction and the preferred direction. The voxel-based tunings were first min-max normalized and then averaged across all voxels in the ROI to create the population tuning function. The average of the population tuning functions in PMT across the ten subjects is plotted. Error bars represent the standard error of the population mean. (C) Gaussian function with four free parameters (of baseline, amplitude, bandwidth and center of the tuning curve) was fitted to the population voxel-based tuning for each subject. The distribution of the feature-based attentional indices is plotted for each fit parameter for pMT voxels. (B) The average of the population voxel-based tunings across ten subjects is plotted in a same way as Fig.8.A for voxels in PMST. (D) Indices of the attention modulation of the fit parameters are shown for pMST tuned voxels in a same way as Fig.8.B. There was a significant modulation of the Gaussian amplitude (median $-12 \%, p=0.03$ Wilcoxon sign ranked test). 
A

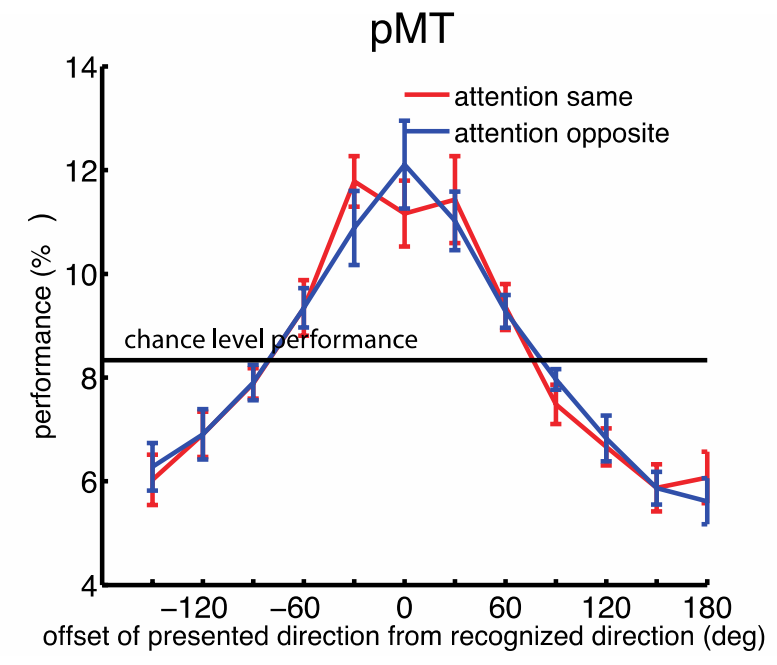

B

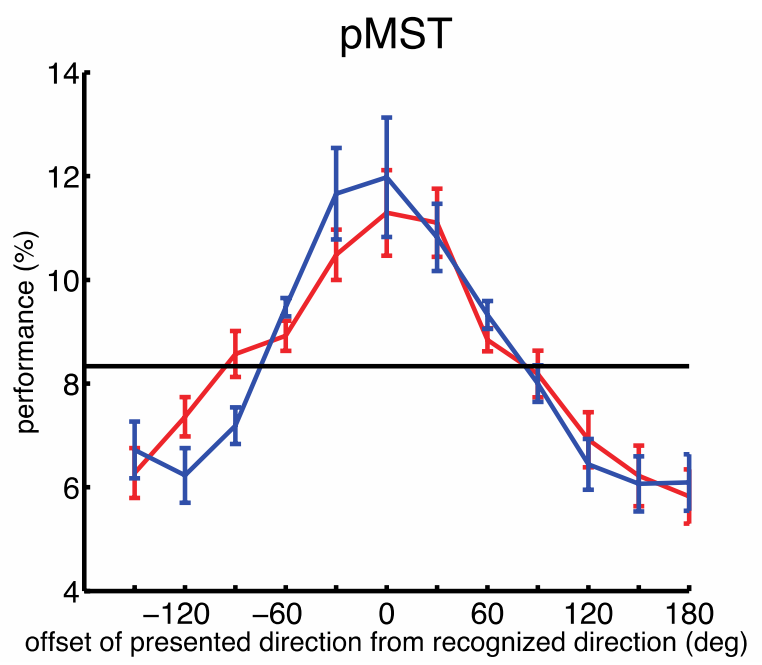

Fig.9. Decoding of the presented spiral with and without feature-based attention

(A) The decoder performance is plotted as a function of the offset between the presented direction and the predicted direction for PMT voxels in same way as the Fig.6. The decoder did not show any improvement of its performance by feature-based attention. (B) The classifier performance was plotted as in panel A for area pMST. There was no significant modulation of the classifier performance by feature-based attention.

\section{DISCUSSION}

We investigated the effects of spatial and feature-based attention on responses to spiral motion patterns in MT and MST subdivisions of the human MT-complex. The results of the first spatial attention experiment show a $13 \%$ and $10 \%$ modulation of the peak response to the expanding spiral patterns in pMT and PMST respectively. We examined voxel-based tuning properties in PMT and pMST in the second experiment. About $39 \%$ of pMT and $33 \%$ of pMST voxels were selected as tuned voxels. Moreover, spatial attention caused an additive modulation of the population voxel-based tuning functions in PMT and a multiplicative modulation of them in pMST. In the third experiment, we investigated the modulatory effects of feature-based attention on the hemodynamic activity. We observed that the peak response decreases with attending to the same direction as the probe stimulus only in PMST whereas no significant effect was seen in PMT. In the fourth experiment, feature-based attentional modulation of the voxel-based tunings was examined. About $20 \%$ of voxels in both PMT and PMST passed our tuning criterion. We then fitted a Gaussian function to their population tuning profile. The 
amplitudes of the fits to the population voxel-based tuning were reduced by feature-based attention in PMST, whereas feature-based attention did not show any influence on the properties of the fitted Gaussians to the population voxelbased tunings in PMT.

\section{Localizer}

In this study we adapted the localizer paradigm of Huk et al. (2002) to identify MT and MST subdivisions of the human MT complex based on their difference to activate the ipsilateral part of the MT complex. Although, the main distinguishable difference of MT and MST is known to be their receptive field size (Huk et al., 2002; Smith et al., 2006), it is still possible that we had erroneously misidentified some part of MT as MST or vice versa. Therefore, we repeated the analysis for investigating the effect of spatial and feature-based attention on the hemodynamic responses to the spiral motion patterns, using the averaged Talairach coordinates of MT and MST reported by Kolster et al. (2010). The observed attentional modulation showed a similar trend as the one we identified using our defined ROls, though using different ROls removed the significance of our results (spatial attention: Sup-Fig.4, feature-based attention: Sup-Fig. 5).

The average coordinates of the localized pMT and PMST were inferior to the coordinates previously (Dukelow et al., 2001; Kolster et al., 2010; Smith et al., 2006) but approximately at the same location as what was reported as the specialized part of human MT-complex for optic flow processing (de Jong et al., 1994; Morrone et al., 2000). Either this inconsistency is related to physical differences between the stimuli that the two kinds of localizers used or it might occur because of the variation induced by the normalization to the Talairach space. Moreover, using spiral stimuli with a speed gradient rather than conventional stimuli without heading component, we might activate the inferior satellite of the MT-complex rather than its conventional MT and MST subdivisions. The inferior satellite of the MT-complex has been suggested as the most probable human homologue for MSTd in monkeys (Peuskens et al., 2001). However, a consensus on identifying the human homologues of all monkey areas has not been reached yet.

\section{Voxel-based tunings to spiral motion patterns}

Many monkey electrophysiological studies have shown existence of selectivity to different feature dimensions across the visual cortex. For example, V1 shows 
tuning to the orientation of bars (Hubel and Wiesel, 1968). Further along the dorsal stream of the monkey's visual cortex, area MT is selective to the linear motion direction (Albright, 1984; Dubner and Zeki, 1971; Maunsell and Van Essen, 1983b) and higher in the hierarchy, MSTd is tuned to the direction of the spiral motion patterns (Graziano et al., 1994). In this hierarchical processing, each area seems to be involved in processing one feature of the incoming visual information as its main feature, although they show selectivity to other features as well (Van Essen and Maunsell, 1983). Moreover, complexity of the encoded features seems to increase in higher areas along the visual hierarchy (Van Essen and Maunsell, 1983). We investigated the encoding of spiral motion patterns in the BOLD signal in PMT and PMST as an indirect evidence for neuronal tuning in those areas. Based on our inclusion criterion about $30 \%$ of voxels were selected as tuned voxels in both pMT and pMST in the spatial attention experiment and about $20 \%$ of voxels in the feature-based attention experiment. The observed voxel-based tunings within a proportion of MT/MST voxels might reveal the existence of neuronal spiral tuning in the human MT complex under the assumption of an anisotropic distribution of such neuronal tunings within tuned voxels, which could be an evidence for columnar organization in human MT-complex. The observed voxel-based tuning to spiral motions was expected in PMST based on electrophysiological studies in macaque monkeys (Graziano et al., 1994). The observed tuning in pMT could relate to the hypothetical feedback connections from human MST to MT, which were demonstrated in macaque monkey's visual cortex (Maunsell and Van Essen, 1983a). Moreover, the detected voxel-based tunings might emerge from the summation of the tuning to linear components of the spiral stimulus across the population of neurons within each voxel. However, this suspicion seems unlikely to be true for PMST because of its large receptive fields. In this case, feature-selective attentional modulation can reveal the preference of each area for a specific dimension of the stimulus. As we observed featureselective attentional modulation (both spatial and feature-based attentional effects) only in PMST, we can conclude that spiral motion seems to be the preferred dimension for human MST.

There was an inherited spatial smoothing in our analysis as we transferred the functional scans into the anterior commissure-posterior commissure (AC-PC) plane and the standard Talairach space. This translation induced some spatial 
smoothing because of all translation, rotation and scaling steps. This induced smoothing could potentially attenuate the voxel-based tunings and increase the false-negative error rate of our suggested inclusion criterion. Thus, there might be more tuned voxels in pMT/pMST, which were rejected to be a tuned voxels. Although the spatial smoothing may attenuate the tuning, it may also increase the SNR in each voxel by canceling out the spatial noise caused by movement.

Furthermore, we checked the sanity of our suggested inclusion criterion by replacing the BOLD signal of each voxel with random values with normal or Poisson distribution and then checked our inclusion criterion using these noisy voxels, where less than $5 \%$ of them were selected as tuned voxels.

\section{Spatial attention}

Attending to a target stimulus in a specific part of the visual field enhances the activity of neurons responding to it. Such a spatial attention modulation was shown to be more pronounced in the higher areas along the visual hierarchy (Maunsell and Cook, 2002; Treue and Maunsell, 1999). We examined the attentional modulation of responses to spiral motion patterns in PMT and PMST. We used a deconvolution analysis to estimate the hemodynamic response in our rapid eventrelated design in each attentional condition. We observed spatial attention enhancing the peak of the hemodynamic response to expanding spiral stimuli in both pMT (13\%) and pMST (10\%) subdivision of MT complex. Although, the similar magnitudes of the modulations in both areas were in disagreement with previous studies (Maunsell and Cook, 2002; Treue and Maunsell, 1999), we cannot conclude that attention modulates the BOLD signal similarly in MT and MST. Indeed, this observed modulation could be because of the bias of our localizer in identifying voxels with big population receptive fields as PMST voxels. In other words, our spatial attention contrast (attention-contra vs. attention-ipsi) for pMT voxels is more or less equal to comparing attention-inside vs. attentionoutside of the population receptive fields; though it is not applicable for PMST voxels. Actually, we identified PMST as part of MT-complex, which is responding both contralaterally and ipsilaterally to the stimulus, with a lack of retinotopic organization. Therefore, the spatial attention contrast is mostly comparing attention-to-the-receptive-field-hotspot vs. attention-to-the-far-boundaries. Given together, the observed attentional modulation in PMST supports attentional modulation within receptive fields. Moreover, given this inconsistency in spatial 
attention contrast, we cannot compare the order of the modulation magnitude in PMT versus PMST.

Electrophysiological studies demonstrated that spatial attention is modulating the neuronal activity in a stimulus-selective fashion; i.e. there is a stronger increase in the neuronal firing rate for the preferred stimulus than the increase for the null stimulus (McAdams and Maunsell, 1999; Treue and Maunsell, 1996). Such a stimulus-selective modulation would be reflected as a multiplicative modulation of the tuning functions and consequently as a multiplicative modulation of the population voxel-based tuning. So far, our knowledge about the influence of attention on the population response profiles to spiral motion patterns has been restricted due to technical limitations inherited in recording/imaging techniques. Electrophysiological recording restricts the measurement to a very small cortical region. In contrast, functional imaging techniques such as $\mathrm{FMRI}$ could potentially reveal voxel-based tunings (Saproo and Serences, 2010; Serences et al., 2009). By decreasing the voxel-size and with the help of analytical methods, we examined voxel-based tunings and the effects of spatial attention on tuning properties in PMT and PMST. We applied an inclusion criterion to select tuned voxels for further examination of attentional modulation. We then investigated the influence of attention on the extracted voxel-based tuning profiles and observed a $90 \%$ additive modulation in PMT and $8 \%$ multiplicative modulation in PMST. If the voxel-based tuning properties are weak in pMT, then any multiplicative modulation could be seen as an additive scaling. This could occur under measurement variability when the mean standard error of the amplitude modulation is bigger than its mean. In this case, the observed additive attentional modulation of PMT voxel-based tuning could be in fact a week multiplicative modulation, which was misidentified as additive modulation. Thereby, we expect to see a positive correlation between the multiplicative modulation and the selectivity of the tuned voxels. Sup.Fig. 6 depicts the scatter plot of the multiplication modulations versus the selectivity indices for all tuned voxels in area pMT for a sample subject. We did not observe any positive correlation between the attentional modulation and the selectivity of the voxels. The same result was observed for the other subjects. Therefore, we could confirm the validity of the observed additive modulation. We also applied Monte Carlo cross validation to ensure that the tuning properties as well as the attentional modulation are not due to noise or any bias in selecting 
training-test data sets; as the noise effects should be canceled out by the cross validation. Together, the lack of stimulus-selective attentional modulation in pMT shows that spiral motion is a non-preferred feature for human MT, whereas, the observed multiplicative feature-selective modulation in PMST indicates spiral motion as a preferred feature for human MST.

Moreover, multiplicative attentional modulation observed in PMST could increase the information content of the voxel-based tunings by increasing the dynamic range of them, which eventually might facilitate decoding of features in higher areas (Butts and Goldman, 2006; Saproo and Serences, 2010). In comparison, additive modulation is supposed to enhance the signal to noise ratio at single unit level, since noise is scaled with the root of signal response ( $\Delta$ noise $\propto \Delta \sqrt{\text { signal })}$ under the assumption of Poisson noise (typical neuronal noise in visual cortex) (Mitchell et al., 2007). Even though additive scaling is assumed to increase the signal to noise ratio at single unit level, its role is not very clear at the population level yet (Saproo and Serences, 2010). Therefore, we cannot indicate the role of additive modulation observed in the tuned voxels in pMT in perception of spiral patterns. Thus, we examined the effect of attention on perception of spiral stimuli by proposing a neuronal decoder indirectly. Neuronal coding is important, as it seems to contribute to visual perception (Kamitani and Tong, 2005, 2006). In the second experiment, tuned voxels with different preferred directions can form distinctive activity patterns distributed in MT/MST, which eventually yield decoding of different spiral directions. There, attention could bias such neuronal coding in favor of behaviorally relevant stimuli (Kamitani and Tong, 2006; Saproo and Serences, 2010; Serences et al., 2009). Therefore, we suggested a decoder, which is combining the information across all tuned voxels to link BOLD responses to different spiral stimuli. Our decoder was successful to predict the direction of the presented stimulus with accuracy of about $12 \%$, which is better than chance level. We did not observe any improvement of the performance by attention in PMST but in PMT. The lack of significant attentional modulation of the decoder performance in PMST could be due to the small magnitude of the multiplicative attentional modulation, where a higher magnitude of the modulation is necessary to increase the tunings dynamic range and accordingly improve in the classifier performance in pMST. And it might also be due to the decoder's low power to determine the presented direction. 


\section{Feature-based attention}

Attending to a specific feature of the target stimulus, such as its motion direction, increases the activity of neurons coding that specific feature across the whole visual field as a global selective mechanism (Maunsell and Treue, 2006; Saenz et al., 2002). We investigated the effect of feature-based attention on the hemodynamic responses to the spiral motion patterns using deconvolution analysis in the rapid event-related design. Our results demonstrated a $-3 \%$ modulation of the peak of the hemodynamic response with feature-based attention only in PMST subdivision of MT-complex. This suppression of the hemodynamic activity with feature-based attention is in disagreement with previously reported results (Martinez-Trujillo and Treue, 2004; Stoppel et al., 2011).

We also examined the effect of the feature-based attention on the population voxel-based tunings. We first applied an inclusion criterion to select tuned voxels, where about $20 \%$ of pMT and pMST voxels were accepted. Investigating the influence of feature-based attention on the voxel-based tuning properties, we observed $-12 \%$ multiplicative modulations of the fitted Gaussians to the voxelbased tunings in PMST. We saw no feature-based attentional modulation of the fits parameters in PMT, which is consistent with our observed hemodynamic peak modulation reported before.

In our experimental paradigms, feature-similarity gain model predicts a selectivity modulation of the population voxel-based tunings, while the feature-matching model, as a feature independent mechanism, expects additive modulation. If we assume a positive relationship between the BOLD signal and the neuronal firing rate, both models expect a positive modulation of the tuning profiles, which is in disagreement with our observed negative modulation. Therefore, the negative observed modulation could be presumably because one of the following possibilities:

- As the stimulus with same direction was always located at the upper visual field and the opposite stimulus at the lower field, one could argue that the modulation is because of the spatial attention directed to the upper versus lower visual field. We investigated the feature-based attentional modulation in voxels ipsilateral to the focus of spatial attention. Thus, if spatial attention caused the observed effect, we would expect to see higher or at least same order of modulation magnitude in voxels contralateral to the spatial attention. Therefore, we 
calculated the hemodynamic response as well as the voxel-based tuning modulations for the contrast of attention-up vs. attention-down for the contralateral voxels to the spatial attention. We observed significant modulation neither in PMT nor in PMST in the both feature-based attention experiments (Sup.Fig.7, Sup.Fig.8). Moreover, it was already shown that the spatial attention in MTcomplex has no retinotopic organization, i.e. attending to the upper versus the lower visual field cannot cause any significant modulation (Tootell et al., 1998). Thus, we can rule out that attending to upper vs. lower stimulus causes the observed effects.

- It is also possible that the actual feature-based attention happened at neighboring areas to PMST in the MT-complex and the observed negative modulation in PMST was because of the blood stealing.. Therefore, we checked the feature-based attention modulation in the pre-localized MT-complex subregions as well as its neighboring areas reported by (Kolster et al., 2010) as shown in Sup.Fig.5. We observed no significant modulation in any of the neighboring areas to MT and MST, however, we observed positive modulation in the posterior inferior temporal (PIT) region. This area is a part of the visual ventral stream, which is located in IT cortex. PIT seems to code the contrast of 3D object versus 2Ds (Kolster et al., 2010). There is also some evidence that it might combine motion signal with object information (Thomas Yeo et al., 2011). Therefore, as spiral patterns are somehow appearing as 3D moving stimuli, it might be possible that attending to different types of spiral patterns elicits featurebased attentional modulation in area PIT. We could also speculate that the observed modulation in PIT originated from the hypothetical connections from MTcomplex to PIT.

- It is possible to argue that positive modulation in tuned voxels in PMST caused blood stealing from other non-tuned voxels, and ultimately yielded the negative modulation in PMST. We could reject this speculation, as we observed negative multiplicative modulation even in the tuned voxels in pMST (Fig.8).

- In our experimental paradigm, we had one stimulus with opposite direction than the other two stimuli. This stimuli configuration might create a pop-out singleton feature, which might be more salient than the others might. Thus, attending to such a salient feature could modulate the neuronal activity of neurons contributing to its encoding (Müller et al., 1995). Thereby, we would see a positive 
modulation by attending to the singleton opposite direction, consistent with what we observed. Although the singleton feature modulation justifies our results obtained from the ipsilateral voxels to the spatial attention, it falls short to explain the lack of such a modulation in contralateral voxels.

All together, unfortunately we failed to provide explanation for the mechanism behind the resultant modulation observed in our two feature-based attention experiments.

The increased dynamic range caused by attending to the opposite direction in PMST could increase the information content of the voxel-based tunings, and eventually might facilitate spiral direction decoding (Butts and Goldman, 2006; Saproo and Serences, 2010). Our proposed decoder was successful to predict the direction of the presented stimulus with about $12 \%$ accuracy, which was better than the chance level. However, feature-based attention did not to improve the performance of our decoder either in PMST or in PMT. The small magnitude of the multiplicative modulation besides the small number of tuned voxels (about 20\%) might not provide our decoder with enough power to significantly improve its performance.

\section{CONCLUSION}

In this study, we showed voxel-based tunings to spiral motion patterns in a subpopulation of pMT and pMST voxels ( $30 \%$ of the voxels in the spatial attention experiment and $\sim 20 \%$ of the voxels in the feature-based attention experiment). This result confirms the existence of neuronal tuning to spiral motions in the human MT-complex, similar to what has been shown in area MSTd of monkeys (Graziano et al., 1994).

The observed spatial attention modulation in PMST might add to the evidences for spatial attention modulation within receptive fields, as some of the receptive fields in MST include the unattended stimulus in the ipsilateral visual field, contrary to the receptive fields in MT. On the other hand, this important differences between the attentional contrasts in PMT and PMST limits us to compare the attentional modulatory effects quantitatively.

The observed multiplicative spatial attention modulation in PMST is in agreement with the electrophysiological studies reporting stimulus-specific modulation at the 
neuronal level. However, the absence of such a multiplicative modulation in PMT indicates that spiral motion is not the preferred dimension in human pMT.

We observed a suppressive feature-based attention modulation of hemodynamic activity in pMST. Similarly, we saw a suppressive multiplicative modulation of the Gaussian fits to the voxel-based tunings only in PMST. These results indicate an area-specific feature-based modulation, which suggested pMST but not pMT as the area with most contribution to the spiral motion encoding and consequently spiral motion processing. Furthermore, we cannot explain the observed modulation of the BOLD in the feature-based attention experiments by the featuresimilarity gain model. Thus, we could not determine the mechanisms behind the observed modulation of the BOLD signal in PMST in our feature-based attention experiments. 


\section{BIBLIOGRAPHY}

Albright, T.D., 1984. Direction and orientation selectivity of neurons in visual area MT of the macaque. Journal of Neurophysiology 52, 1106-1130.

Albright, T.D., Desimone, R., 1987. Local precision of visuotopic organization in the middle temporal area (MT) of the macaque. Experimental Brain Research 65, 582-592.

Buracas, G.T., Boynton, G.M., 2007. The effect of spatial attention on contrast response functions in human visual cortex. Journal of Neuroscience 27, 93-97.

Butts, D.A., Goldman, M.S., 2006. Tuning curves, neuronal variability, and sensory coding. PLoS Biology 4, 0639-0646.

Cao, F., Hong, W., Wu, Y., Pottier, E., 2007. The Comparison of the V-Fold and the Monte-Carlo cross validation to estimate the number of clusters for the fully polarimetric sar data segmentation, Geoscience and Remote Sensing Symposium, 2007. IGARSS 2007. IEEE International, pp. 2485-2486.

Carrasco, M., McElree, B., 2001. Covert attention accelerates the rate of visual information processing. Proceedings of the National Academy of Sciences 98, 5363-5367.

Cook, E.P., Maunsell, J.H.R., 2004. Attentional modulation of motion integration of individual neurons in the Middle Temporal visual area Journal of Neuroscience 24, 79647977.

Dale, A.M., Buckner, R.L., 1997. Selective averaging of rapidly presented individual trials using fMRI. Hum Brain Mapp 5, 329-340.

de Jong, B.M., Shipp, S., Skidmore, B., Frackowiak, R.S.J., Zeki, S., 1994. The cerebralactivity related to the visual-perception of forward motion in-depth. Brain 117, 1039-1054.

Desimone, R., Duncan, J., 1995. Neural mechanisms of selective visual attention. Annual Review of Neuroscience 18, 193-222.

Desimone, R., Ungerleider, L.G., 1986. Multiple visual areas in the caudal superior temporal sulcus of the macaque. Journal of Comparative Neurology 248, 164-189.

Dubner, R., Zeki, S.M., 1971. Response properties and receptive fields of cells in an anatomically defined region of the superior temporal sulcus in the monkey. Brain Research 35, 528-532.

Duffy, C.J., Wurtz, R.H., 1991a. Sensitivity of MST neurons to optic flow stimuli. I. A continuum of response selectivity to large-field stimuli. Journal of Neurophysiology 65, 1329-1345.

Duffy, C.J., Wurtz, R.H., 1991b. Sensitivity of MST neurons to optic flow stimuli. II. Mechanisms of response selectivity revealed by small-field stimuli. Journal of Neurophysiology 65, 1346-1359.

Dukelow, S.P., De Souza, J.F.X., Culham, C.J., Van den Berg, A.V., Menon, R.S., Vilis, T., 2001. Distinguishing subregions of the human MT+ complex using visual fields and pursuit eye movements. Journal of Neurophysiology 86, 1991-2000.

Eriksen, C.W., Yeh, Y., 1985. Allocation of attention in the visual field. Journal of Experimental Psychology: Human Perception and Performance 11, 582-597. 
Gandhi, S.P., Heeger, D.J., Boynton, G.M., 1999. Spatial attention affects brain activity in human primary visual cortex. Proceedings of the National Academy of Sciences 96, 33143319.

Graziano, M.S.A., Andersen, R.A., Snowden, R.J., 1994. Tuning of MST neurons to spiral motions. Journal of Neuroscience 14, 54-67.

Hubel, D.H., Wiesel, T.N., 1968. Receptive fields and functional architecture of the monkey striate cortex. Journal of Physiology 195, 215-243.

Huk, A.C., Dougherty, R.F., Heeger, D.J., 2002. Retinotopy and functional subdivision of human areas MT and MST. Journal of Neuroscience 22, 7195-7205.

Kamitani, Y., Tong, F., 2005. Decoding the visual and subjective contents of the human brain. Nature Neuroscience 8, 679-685.

Kamitani, Y., Tong, F., 2006. Decoding seen and attended motion directions from activity in the human visual cortex. Current Biology 16, 1096-1102.

Kolster, H., Peeters, R., Orban, G.A., 2010. The retinotopic organization of the human middle temporal area MT/V5 and its cortical neighbors. Journal of Neuroscience 30, 98019820.

Komatsu, H., Wurtz, R.H., 1988. Relation of cortical areas MT and MST to pursuit eye movements I. Localization and visual properties of neurons. Journal of Neurophysiology $60,580-603$.

Liu, T., Larsson, J., Carrasco, M., 2007. Feature-based attention modulates orientationselective responses in human visual cortex. Neuron 55, 313-323.

Martinez-Trujillo, J.C., Treue, S., 2004. Feature-based attention increases the selectivity of population responses in primate visual cortex. Current Biology 14, 744-751.

Maunsell, J.H.R., Cook, E.P., 2002. The role of attention in visual processing. Philosophical Transactions of the Royal Society of London, Series B 357, 1063-1072.

Maunsell, J.H.R., Newsome, W.T., 1987. Visual processing in monkey extrastriate cortex. Annual Review of Neuroscience 10, 363-401.

Maunsell, J.H.R., Treue, S., 2006. Feature-based attention in visual cortex. Trends in Neurosciences 29, 317-322.

Maunsell, J.H.R., Van Essen, D.C., 1983a. The connections of the middle temporal visual area (MT) and their relationship to a cortical hierarchy in the macaque monkey. Journal of Neuroscience 3, 2563-2586.

Maunsell, J.H.R., Van Essen, D.C., 1983b. Functional properties of neurons in the middle temporal visual area (MT) of the macaque monkey. I. Selectivity for stimulus direction, speed, and orientation. Journal of Neurophysiology 49, 1127-1147.

McAdams, C.J., Maunsell, J.H.R., 1999. Effects of attention on orientation-tuning functions of single neurons in Macaque cortical area V4. Journal of Neuroscience 19, 431 441.

Mitchell, J.F., Sundberg, K.A., Reynolds, J.H., 2007. Differential attention-dependent response modulation across cell classes in Macaque visual area V4. Neuron 55, 131-141. 
Morrone, M.C., Tosetti, M., Montanaro, D., Florentinii, A., Cioni, G., Burr, D.C., 2000. A cortical area that responds specifically to optic flow, revealed by fMRI Nature Neuroscience 3, 1322-1328.

Motter, B.C., 1994. Neural correlates of attentive selection for color or luminance in extrastriate area V4. Journal of Neuroscience 14, 2178-2189.

Müller, H.J., Heller, D., Ziegler, J., 1995. Visual search for singleton feature targets within and across features dimensions. Perception \& Psychophysics 57, 1-17.

Murray, S.O., Wojciulik, E., 2004. Attention increases neural selectivity in the human lateral occipital complex. Nature Neuroscience 7, 70-74.

O'Craven, K.M., Rosen, B.R., Kwong, K.K., Treisman, A., Savoy, R.L., 1997. Voluntary attention modulates fMRI activity in human MT-MST. Neuron 18, 591-598.

O'Craven, K.M., Savoy, R.L., 1995. Attentional modulation of activation in human MT shown with functional magnetic resonance imaging (fMRI), Association for the Research in Ophthalmology (USA), Annual Meeting, p. S856.

Pestilli, F., Carrasco, M., 2005. Attention enhances contrast sensitivity at cued and impairs it at uncued locations. Vision Research 45, 1867-1875.

Peuskens, H., Sunaert, S., Dupont, P., Van Hecke, P., Orban, G.A., 2001. Human brain regions involved in heading estimation. The Journal of neuroscience : the official journal of the Society for Neuroscience 21, 2451-2461.

Posner, M.I., 1980. Orienting of attention. Quarterly Journal of Experimental Psychology 32, 3-25.

Posner, M.I., Snyder, C.R.R., Davidson, B.J., 1980. Attention and the detection of signals. JEP-General 109, 160-174.

Rosen, B.R., Buckner, R.L., Dale, A.M., 1998. Event-related functional MRI: Past, present, and future. Proceedings of the National Academy of Sciences 95, 773-780.

Saenz, M., Buracas, G.T., Boynton, G.M., 2002. Global effects of feature-based attention in human visual cortex. Nature Neuroscience 5, 631-632.

Saenz, M., Buracas, G.T., Boynton, G.M., 2003. Global feature-based attention for motion and color. Vision Research 43, 629-637.

Saproo, S., Serences, J.T., 2010. Spatial attention improves the quality of population codes in human visual cortex. Journal of Neurophysiology 104, 885-895.

Serences, J.T., 2011. Mechanisms of selective attention: response enhancement, noise reduction, and efficient pooling of sensory responses. Neuron 72, 685-687.

Serences, J.T., Boynton, G.M., 2007. Feature-based attentional modulations in the absence of direct visual stimulation. Neuron 55, 301-312.

Serences, J.T., Saproo, S., 2010. Population response profiles in early visual cortex are biased in favor of more valuable stimuli. Journal of Neurophysiology 104, 76-87.

Serences, J.T., Saproo, S., Scolari, M., Ho, T., Muftuler, L.T., 2009. Estimating the influence of attention on population codes in human visual cortex using voxel-based tuning functions. Neuroimage 44, 223-231. 
Sheinberg, D.L., Logothetis, N.K., 2001. Noticing familiar objects in real world scenes: the role of temporal cortical neurons in natural vision. Journal of Neuroscience 21, 1340-1350.

Smith, A.T., Wall, M.B., Williams, A.L., Singh, K.D., 2006. Sensitivity to optic flow in human cortical areas MT and MST. European Journal of Neuroscience 23, 561-569.

Somers, D.C., Dale, A.M., Seiffert, A.E., Tootell, R.B.H., 1999. Functional MRI reveals spatially specific attentional modulation in human primary visual cortex. Proceedings of the National Academy of Sciences 96, 1663-1668.

Stoppel, C.M., Boehler, C.N., Strumpf, H., Heinze, H.J., Noesselt, T., Hopf, J.M., Schoenfeld, M.A., 2011. Feature-based attention modulates direction-selective hemodynamic activity within human MT. Human Brain Mapping 32, 2183-2192.

Tanaka, K., Saito, H.-A., 1989. Analysis of motion of the visual field by direction, expansion/contraction, and rotation cells clustered in the dorsal part of the medial superior temporal area of the macaque monkey. Journal of Neurophysiology 62, 626-641.

Thomas Yeo, B.T., Krienen, F.M., Sepulcre, J., Sabuncu, M.R., Lashkari, D., Hollinshead, M., Roffman, J.L., Smoller, J.W., Zollei, L., Polimeni, J.R., Fischl, B., Liu, H., Buckner, R.L., 2011. The organization of the human cerebral cortex estimated by intrinsic functional connectivity. J Neurophysiol 106, 1125-1165.

Tootell, R.B.H., Hadjikhani, N., Hall, E.K., Marrett, S., Vanduffel, W., Vaughan, J.T., Dale, A.M., 1998. The retinotopy of visual spatial attention. Neuron 21, 1409-1422.

Treue, S., Martinez-Trujillo, J.C., 1999. Feature-based attention influences motion processing gain in macaque visual cortex. Nature 399, 575-579.

Treue, S., Maunsell, J.H.R., 1996. Attentional modulation of visual motion processing in cortical areas MT and MST. Nature 382, 539-541.

Treue, S., Maunsell, J.H.R., 1999. Effects of attention on the processing of motion in macaque middle temporal and medial superior temporal visual cortical areas. Journal of Neuroscience 19, 7591-7602.

Van Essen, D.C., Maunsell, J.H.R., 1983. Hierarchical organization and functional streams in the visual cortex. Trends in Neurosciences, 370-375.

Verghese, P., Kim, Y.J., Wade, A.R., 2012. Attention selects informative neural populations in human v1. Journal of Neuroscience 32, 16379-16390.

Wall, M.B., Lingnau, A., Ashida, H., Smith, A.T., 2008. Selective visual responses to expansion and rotation in the human MT complex revealed by functional magnetic resonance imaging adaptation. European Journal of Neuroscience 27, 2747-2757.

Yeshurun, Y., Carrasco, M., 1999. Spatial attention improves performance in spatial resolution tasks. Vision Research 39, 293-306.

Zeki, S., Watson, J.D.G., Lueck, C.J., Friston, K.J., Kennard, C., Frackowiak, R.S.J., 1991. A direct demonstration of functional specialization in human visual cortex. Journal of Neuroscience 11, 641-649. 


\title{
Supplementary Material for
}

\section{Attentional modulation of human BOLD responses to spiral motion patterns}

\author{
Sepideh Fazeli ${ }^{1}$, Carsten Schmidt-Samoa ${ }^{2}$, Peter Dechent ${ }^{2}$, Stefan Treue ${ }^{1,3}$ \\ ${ }^{1}$ Cognitive Neuroscience Laboratory, German Primate Center, Goettingen, Germany \\ ${ }^{2}$ Department of Cognitive Neurology, University Medicine Goettingen, Goettingen, Germany \\ ${ }^{2}$ Bernstein Center for Computational Neuroscience, Goettingen, Germany
}

\section{SUPPLEMENTARY FIGURES}

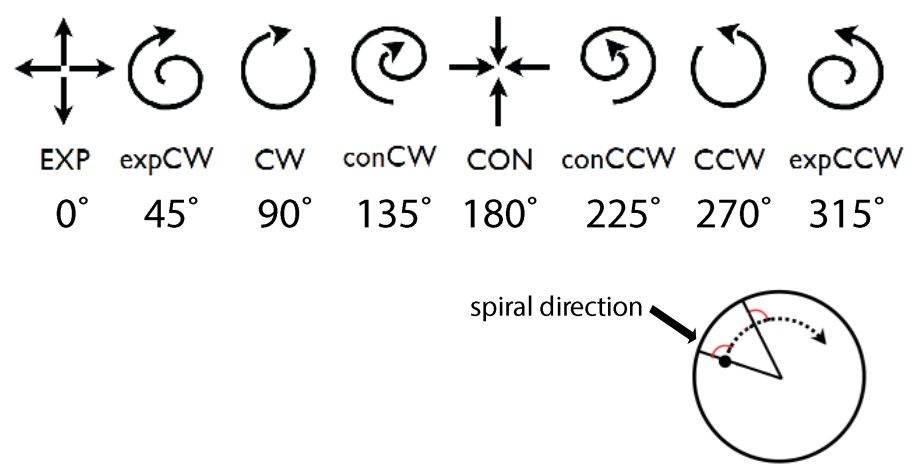

\section{Sup.Fig.1: Trajectory, type and direction (deg) of the spiral motion patterns}

Spiral direction is the angle between the spiral trajectory and its radii. Each spiral direction represents a unique spiral patterns. There, $\phi=0^{\circ}$ is pure expansion (EXP), $0^{\circ}<\phi<90^{\circ}$ corresponds to expanding clockwise spirals, $\phi=90^{\circ}$ is pure clockwise rotation (CW), $90^{\circ}<\phi<180^{\circ}$ defines contracting clockwise spirals RDPs, $\phi=180^{\circ}$ is pure contraction (CON), $180^{\circ}<\varphi<270^{\circ}$ determines contracting counterclockwise spirals RDPs, $\quad \phi=270^{\circ}$ is pure counterclockwise rotation (CCW) and finally $270^{\circ}<\phi<360^{\circ}$ defines expanding counterclockwise spiral RDPs. 

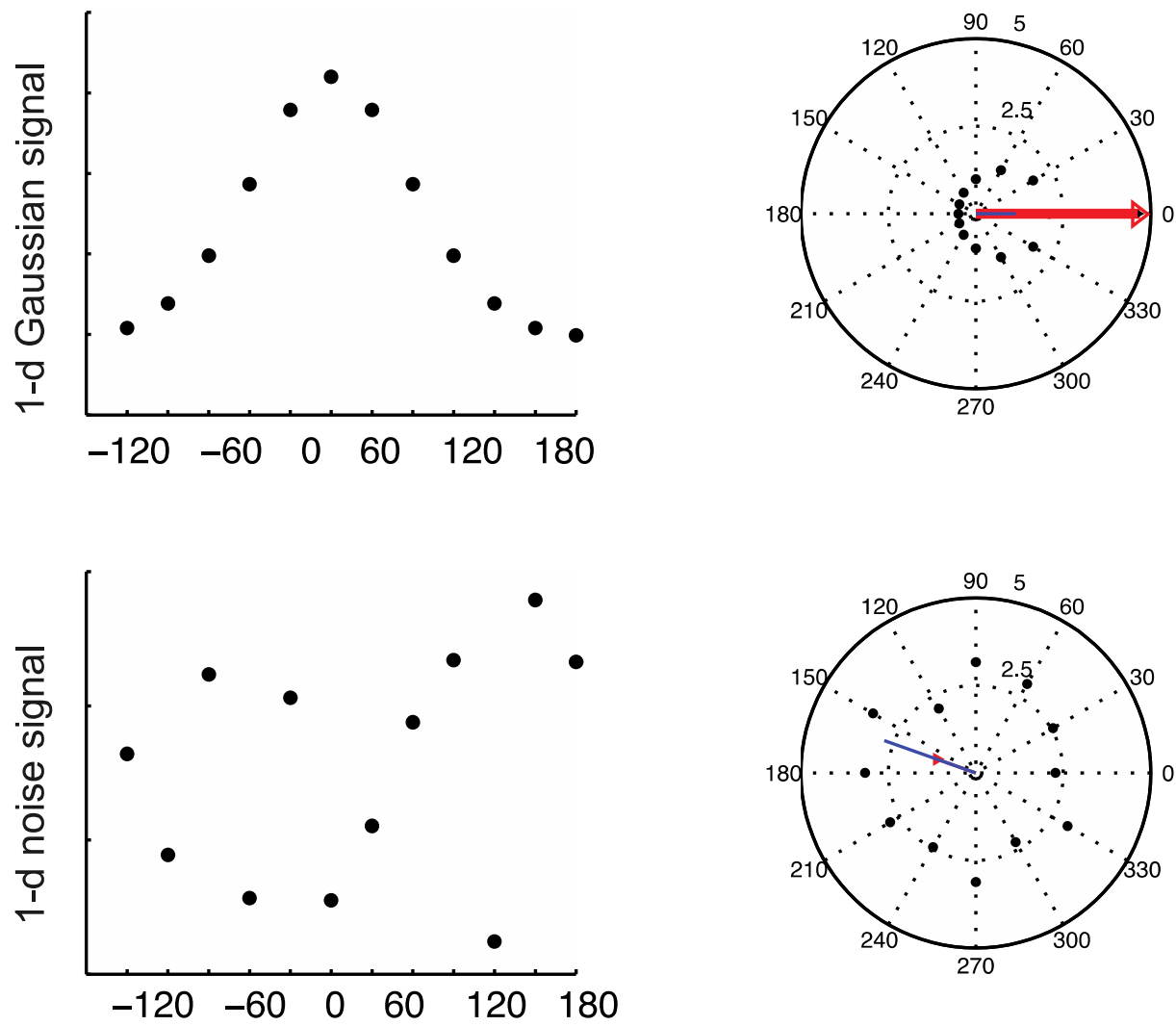

1-d signal

\section{Sup.Fig.2. Selecting tuned voxels versus noise}

A bell-shaped (tuned) response profile and a noisy response function was simulated. Then, the amplitude of their vectorial sum as well as the absolute mean of their response profiles was computed. For a tuned voxel with a bell-shape response profile the magnitude of the vectorial sum is bigger than the absolute mean of its responses to the different directions. In contrast, the magnitude of the vectorial sum of a noisy response profile is smaller compared to its absolute mean. The figure illustrates this difference between tuned and noisy response profiles. 


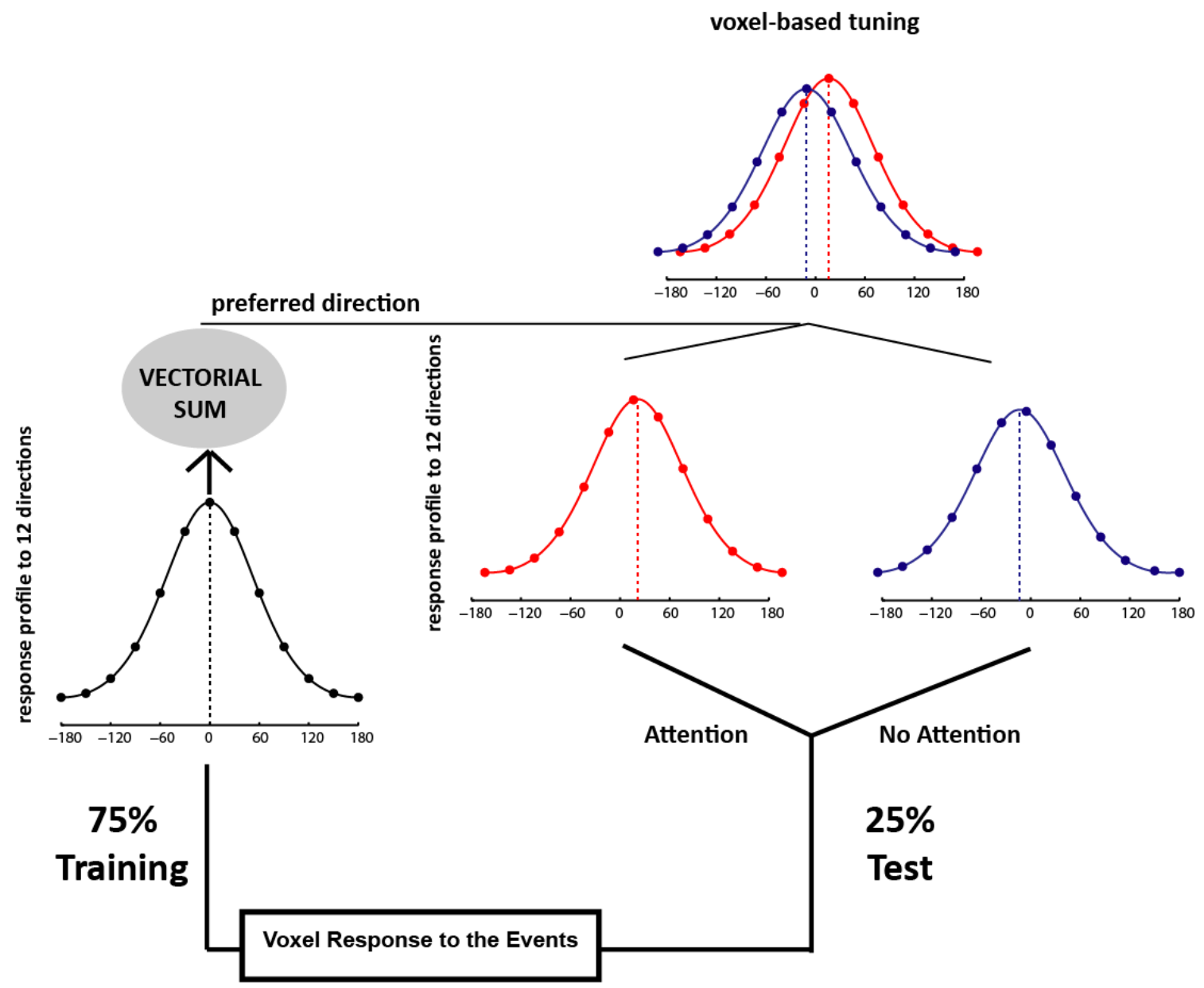

\section{Sup.Fig.3. Flowchart to determine voxel-based tuning}

For each voxel, the BOLD responses to all trials were divided into training ( $75 \%$ of the data) and test $(25 \%$ of the data) datasets. Then the response profiles of each voxel were computed using the training dataset (independent of attention) and the test dataset (for two attention conditions separately). The preferred direction of each voxel was determined by applying vectorial sum to the response profile obtained from the training data. Then, the tuning functions (response profiles) for two attention conditions in the test dataset were aligned to the voxel's preferred direction to deteremine the aligned voxel-based tuning in each attention condition. 


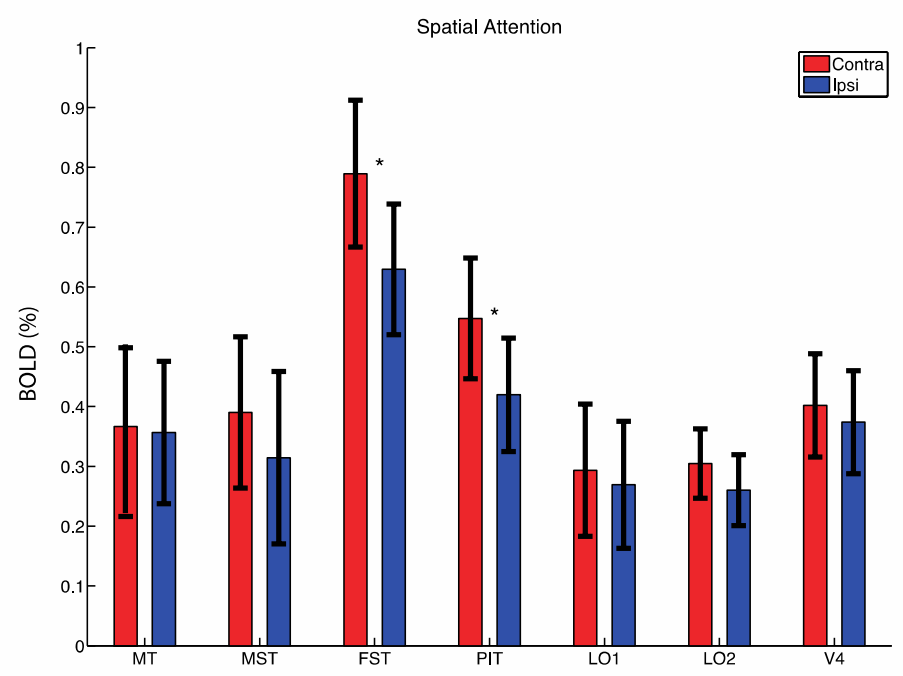

\section{Sup.Fig.4: Spatial attentional modulation in MT-complex and adjacent areas}

Average of the peak hemodynamic responses across eight subjects is plotted for "attend-contra" and "attend-ipsi" conditions in the pre-localized (Kolster et al., 2010) subregions of MT-complex as well as its neighboring areas: MT, MST, fundus of the superior temporal area (FST), posterior inferior temporal area (PIT), lateral occipital 1 (LO1), LO2 and V4. There was a significant difference (Wilcoxon sign ranked test,p<0.05) between the hemodynamic responses in "attendcontra" vs. "attend-ipsi" only in FST and PIT, which is marked with star $\left(^{*}\right)$.

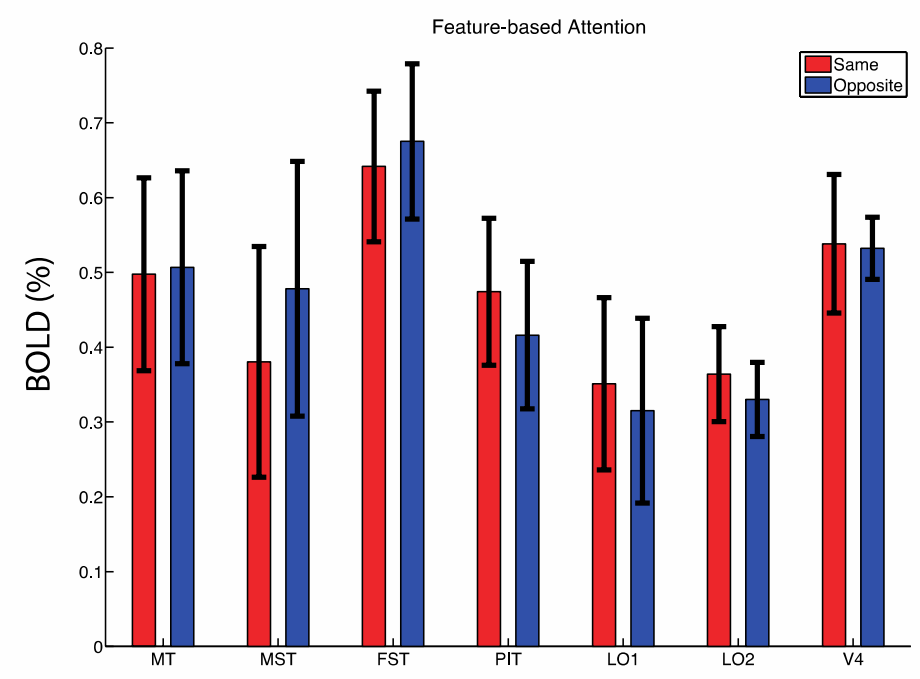

\section{Sup.Fig.5: Feature-based attentional modulation in MT-complex and adjacent areas}

Average of the peak hemodynamic responses across ten subjects is plotted for "attention-same" and "attention-opposite" conditions in the pre-localized MT, MST, FST, PIT, LO1, LO2, V4 (Kolster et al., 2010). There was no significant difference between the hemodynamic responses in these two conditions, though we can see a trend of negative modulation by feature-based attention in MST and a positive trend in PIT while the modulation was significant for PIT-LH (Wilcoxon sign ranked test, $p<0.05)$ 


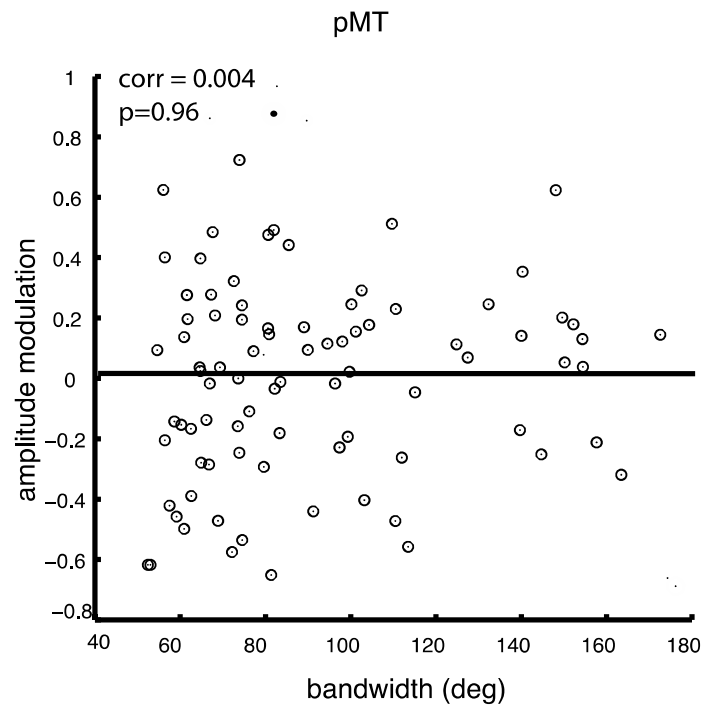

Sup.Fig.6: Amplitude modulation of the voxel-based tunings as a function of their bandwidth in PMT for a sample subject

We fitted a circular Gaussian to the voxel-based tunings in both "attend-contra" and "attend-ipsi" conditions. The amplitude modulation indices are plotted as a function of their corresponding bandwidths in 'attention ipsi'. There was no correlation between the magnitude of the amplitude modulation and the selectivity of the voxel-based tuning (correlation $=0.004, p=0.96$ ). 

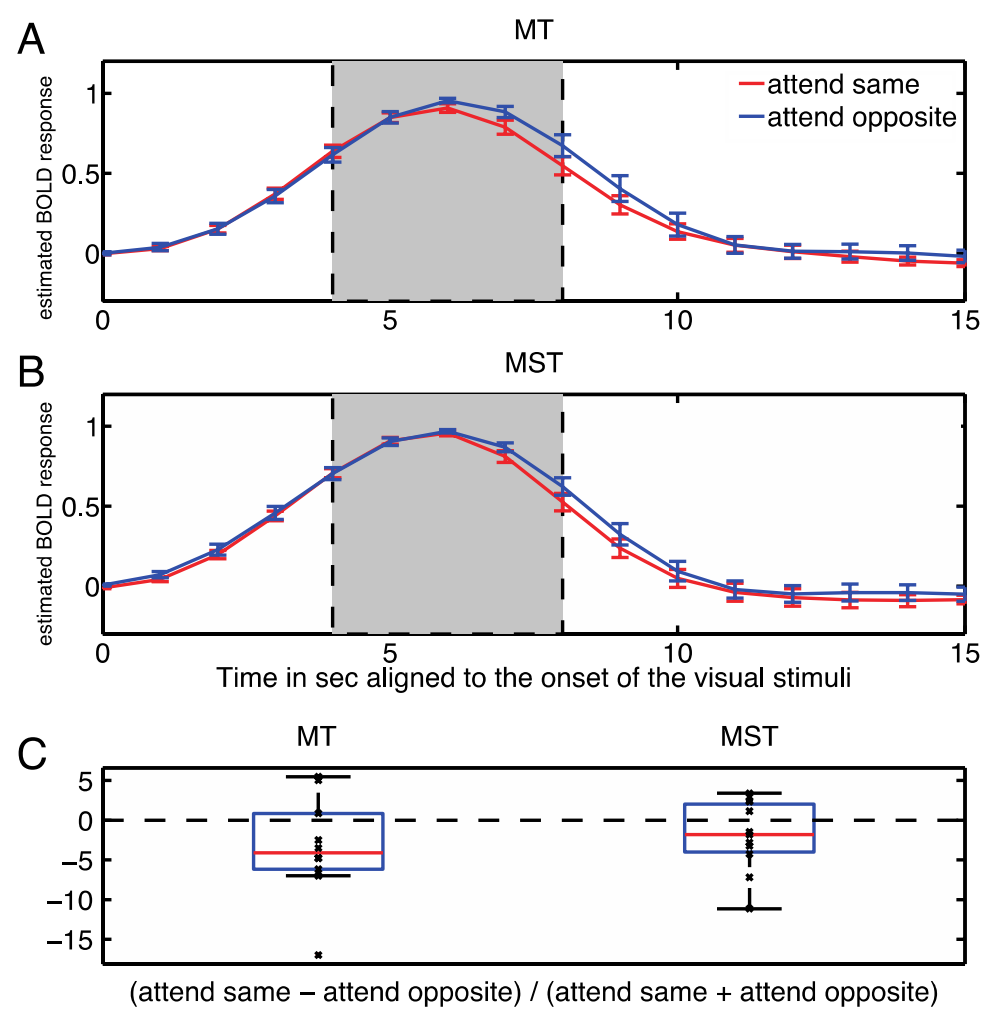

Sup.Fig.7. Modulation of the peak of hemodynamic response to the spiral stimuli in contralateral voxels to the spatial attention in experiment three

(A) Estimated hemodynamic responses to the expanding spiral RDPs were averaged across 10 subjects when attention was directed to the upper conralateral stimulus versus the lower conralateral one in PMT. The error bars represent standard error of the population mean.

(B) The average of the hemodynamic responses in PMST is plotted in a same way as in the Sup.Fig.7.A.

(C) Modulation indices of the peak of hemodynamic responses across the population of ten subjects are plotted for PMT and PMST. No significant modulation was observed either in PMT or in pMST. (Wilcoxon signed rank test, $p>0.5$ ) 
A

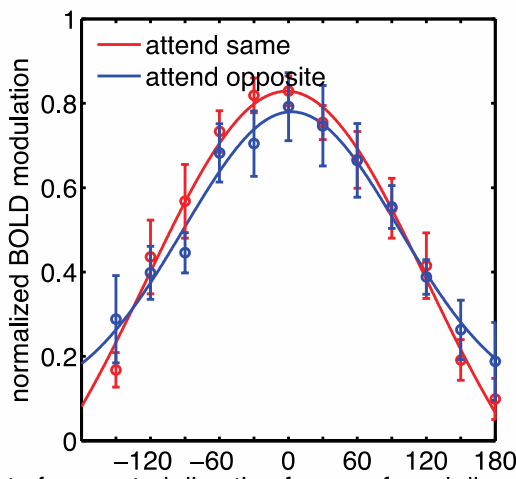

offset of presented direction from preferred direction (deg)

B
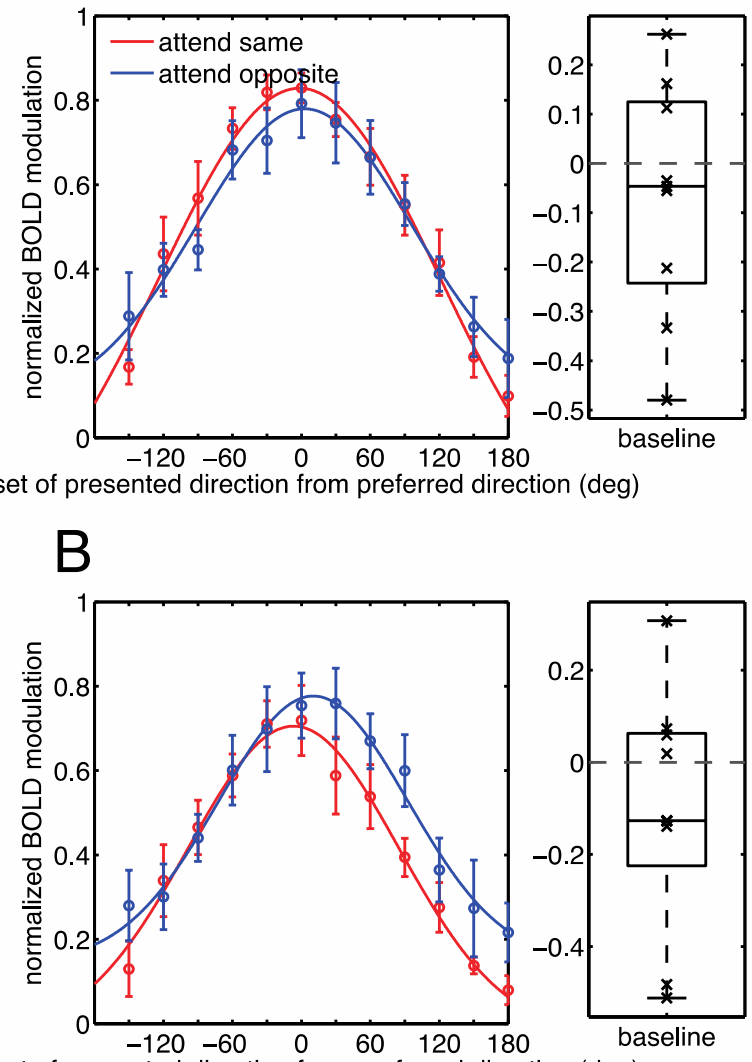
(1)

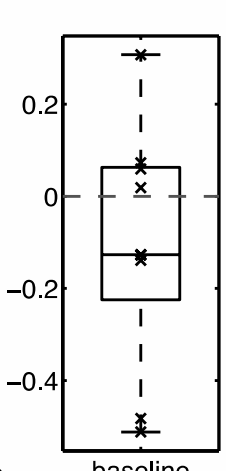

baseline offset of presented direction from preferred direction (deg)
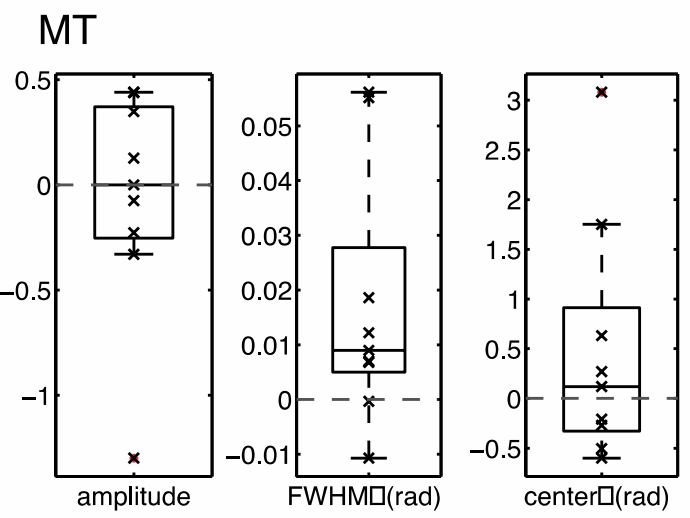

MST
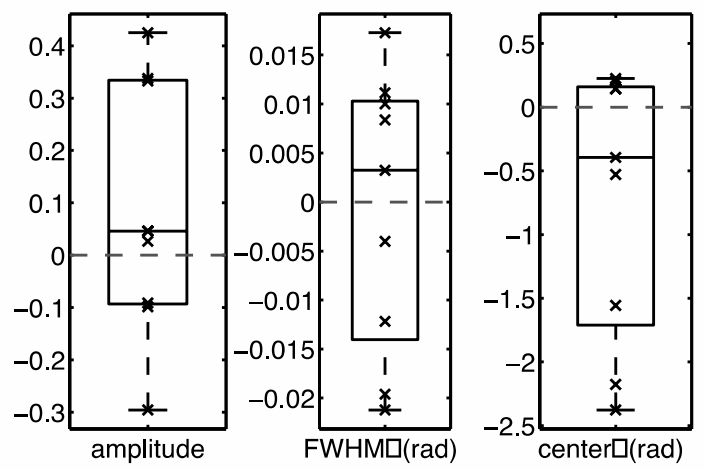

\section{Sup.Fig.8. Attentional modulation of the voxel-based tunings to the spiral stimuli in contralateral visual field}

(A) Average of the population voxel-based tunings across ten subjects when attention was at the upper stimulus and when attention was at the lower one are plotted for the pMT contralateral voxels to the attention task in a same way as Fig. 8.A. Error bars represent the standard error of the population mean.

Modulation indices of the Gaussian fit parameters for pMT voxels are plotted when spatial attention was directed from the lower contralaetral visual field to the upper one. There was no significant modulation (Wilcoxon signed rank test, $p>0.5$ ) of such an attentional shift.

(B) Average of the population voxel-based tunings is plotted in a same way as Sup.Fig.8.A for pMST voxels.

Attentional modulation indices of the fit parameters are plotted for PMST tuned voxels in a same way as Sup.Fig.8.B. We saw no significant modulation of the fit parameters (Wilcoxon signed rank test, $p>0.5)$ 
Sup. Table 1- Averaged Talairach coordinates of the center of the areas in MT/V5 and phPIT cluster and the L01/2 areas in the left and right hemispheres reported by (Kolster et al., 2010)

\begin{tabular}{lcccccc}
\hline & $\mathrm{LH}$ & & & $\mathrm{RH}$ & & \\
Area & $\mathrm{X}$ & $\mathrm{Y}$ & $\mathrm{Z}$ & $\mathrm{X}$ & $\mathrm{Y}$ & $\mathrm{Z}$ \\
MT & -48 & -75 & 8 & 46 & -78 & 6 \\
pMSTV & -45 & -67 & 6 & 44 & -70 & 5 \\
pFST & -46 & -72 & 0 & 46 & -74 & -4 \\
pV4 & -48 & -78 & 3 & 47 & -81 & -2 \\
phPITd & -40 & -85 & -6 & 42 & -85 & -9 \\
phPITv & -39 & -84 & -8 & 40 & -84 & -11 \\
LO1 & -36 & -90 & 4 & 36 & -92 & 3 \\
LO2 & -42 & -89 & -2 & 40 & -91 & -3 \\
& & & & & & \\
\hline
\end{tabular}




\subsection{The influence of spatial attention on human direction discrimination thresholds for spiral motion stimuli}

In this chapter, we investigated the influence of various degrees of spatial attention on the ability of human subjects to discriminate the direction of spiral motion patterns. In order to examine the graded attention, the spiral discrimination thresholds were measured in four attention conditions. Varying the validity of an endogenous pre-stimulus cue created these four attention conditions. Moreover, we systematically altered the strength of the rotational component of the spiral motion pattern to determine the direction discrimination threshold using an adaptive testing paradigm. Hypothetical sensory enhancement caused by attention allocation to the target stimulus set apart from uncertainty reduction induced by pre-cueing the target side using a four-alternative-forced-choice paradigm. Our results indicate a clear pattern for the effect of allocated attention on discrimination performance, which is qualitatively consistent with an attentional strategy where subjects allocate the greatest amount of attention to the most relevant target and the least attention to the least significant one. Furthermore, the observed results suggest that attention substantially affects the ability of humans to accurately perceive the direction of spiral motion stimuli.

\section{Author's contribution}

S. Fazeli and S. Treue designed the experiment. S. Fazeli and L. G. Ramos T. accomplished data collection. S. Fazeli performed the data analysis with the guidance of S. Treue. The manuscript was written by S. Fazeli and edited by S. Treue. All authors discussed the results and worked on the manuscript. 


\title{
The influence of spatial attention on human direction discrimination thresholds for spiral motion stimuli
}

\author{
Sepideh Fazeli ${ }^{1}$, Stefan Treue ${ }^{1,2}$ \\ ${ }^{1}$ Cognitive Neuroscience Laboratory, German Primate Center, Goettingen, Germany \\ ${ }^{2}$ Bernstein Center for Computational Neuroscience, Goettingen, Germany
}

\section{ABSTRACT}

The visual system of primates is equipped with a selection mechanism called attention that enhances behaviorally significant information at the expense of disregarding other irrelevant inputs. Here we investigated the role of graded attention in the discrimination of "spiral motion" patterns by human subjects in two experiments. To examine the effects of attention, the discrimination threshold was measured in a direction discrimination task Subjects had to report whether a briefly shown spiral motion pattern contained clockwise (CW) or counterclockwise (CCW) motion. We systematically varied the strength of the rotational component to determine the direction discrimination threshold. We used a pre-cuing paradigm to alter attention allocation to the target stimulus in both experiments. The relevance of the target side was modified in four grades of $100 \%, 75 \%, 50 \%$ and $25 \%$ (each corresponded to one attention condition) by varying the pre-cue validity in three levels of $100 \%, 75 \%$ and $50 \%$. In both experiments, after pre-cue presentation two stimuli appeared on the screen for $75 \mathrm{~ms}$. One stimulus was a task-relevant probe stimulus, containing spiral random-dot-patterns (RDPs), while the other stimulus was a distracter patch containing spiral noise RDPs. In the exp.1 (only pre-cued), a mask of spiral noise RDPs followed both the target and distracter for $75 \mathrm{~ms}$. In the exp.2 (also post-cued), the probe stimulus was masked by spiral noise RDPs, while the distracter was followed by static dots mask $(75 \mathrm{~ms})$. The static mask can be used as the post-cue indicating the target side. In both experiments, we collected subjects' answer using a four-alternative forced choice (4AFC) paradigm (subjects were asked to report the location and the direction of the target spiral stimulus) to distinguish between the influence of attention allocation and uncertainty reduction caused by pre-cuing the target side. Our results showed a clear pattern for the effect of the attention condition (target relevance) on the discrimination performance. They were qualitatively consistent with an attentional 
strategy where the subjects allocated the greatest amount of attention to the most relevant target, and the least attention to the least relevant stimulus. Moreover, the results suggested that attention substantially affects the ability of humans to perceive spiral motion stimuli accurately.

\section{INTRODUCTION}

The resources of the human visual system are limited to processing only a fraction of all sensory inputs into our sensory systems. Spatial attention is a selection mechanism that allocates more processing resources to relevant spatial locations, at the expense of withdrawing resources from presumably less relevant locations. Hermann Von Helmholtz was the first to show that it is possible to voluntary shift the focus of attention to any location in the visual field without making eye movements (covert spatial attention). He designed an innovative set up for illuminating an array of letters so briefly, that his subjects did not have enough time to foveate them. His subjects were tasked to detect a target letter while they were attending to a pre-cued region. He concluded that the visual performance is related more to the focus of attention than the position of eye fixation (Richard D. Wright, 2008).

Attending to a specific part of the visual field without eye movement defines covert spatial attention. Covert spatial attention has been the foci of many studies, attempting to elucidate the influence of its allocation on the perception of attended stimuli. For example, it was shown that the discriminative ability of the monkeys as well as their neuronal responses in extrastriate cortex to attended stimuli increases when the task demands the allocation of more attention (Spitzer et al., 1988). We can summarize the main conclusions of the previously carried out psychophysics studies of covert attention as: (1) attention can allocate the restricted processing resources to the behaviorally relevant stimuli in order to improve observer performance by increasing the accuracy and processing speed of them compared to if they were neutral for the behavior (Carrasco and McElree, 2001; Yeshurun and Carrasco, 1998, 1999). (2) The cost of such an enhancement at the target is the loss of precision or longer reaction time at those target locations that were less relevant for the task in hand. (Braun and Sagi, 1990; Montagna et al., 2009; Pastukhov et al., 2009; Pestilli and Carrasco, 2005). 
In most of the mainstream studies of attention, there was only one attended target at a time. This kind of attention task is known as the single-task. Nevertheless, there are circumstances in real life when attention has to be allocated to multiple spatially discrete targets simultaneously. Therefore, one of the core questions about the attention system is how the attentional modulation is distributed among multiple locations. This subject has been the focus of many studies to address both its neuronal (Brefczynski and De Yoe, 1999; McMains and Somers, 2004, 2005; Morawetz et al., 2007; Treue and Martinez-Trujillo, 2012) as well as behavioral correlates (Awh and Pashler, 2000; Eriksen and St. James, 1986; Eriksen and Yeh, 1985; Pashler, 1993, 1994; Posner et al., 1980).

Comparing subject's performance on doing two tasks simultaneously (dual task) versus their performance in single-task condition, is one way to investigate attention allocation to multiple targets. It was shown that subject's performance drops in dual-task versus single-task, presumably because multiple targets are interfering with each other (Pashler, 1993, 1994). However, this methodology suffers from some inherited inconsistency across its conditions. For example, in dual discrimination tasks subjects need to respond to both targets at the end of each trail, which adds a memory component to their answers, which is absent in single-task condition.

Furthermore, one of the debates in the field of attention is about the possibility of graded allocation of attention, i.e. whether is it possible to divide attention unevenly between targets based on their behavioral relevance (Dobkins and Bosworth, 2001; Yeshurun and Carrasco, 1999). One of the well-established procedures to orient attention allocation to the targets is pre-cueing paradigm, such as the Posner paradigm (Posner, 1980). The pre-cuing provides subjects with prior information about the target, for example its location. Moreover, the precuing paradigm allows having stimuli with weighted relevance for the task by altering the cue validity in order to examine the graded effect of attention on them. In the study by Posner et al., (1980), subjects were asked to respond as quickly as possible to the onset of a light emitting diode (LED) by pressing a key-operated micro switch. Posner et al. showed that the reaction time of the subjects benefits if the location of the target is pre-cued with $80 \%$ validity. For the invalidly cued $(20 \%$ validity) location, they measured longer reaction times as compared to a neutral condition. This finding along with several other results obtained from different 
studies (e.g. Bashinski and Bacharach, 1980; Doricchi et al., 2010; Fuller et al., 2009; Giordano et al., 2009; Sperling and Melchner, 1978) examining attention allocation by altering cue-validity, leads to the conclusion that performance benefits when a highly valid cue is presented to the subjects. This result is commonly interpreted as the result of a signal enhancement at the target location by orienting spatial attention to it. However, pre-cueing the target location can also reduce the effect of distracters, which ultimately yields uncertainty reduction at the level of decision-making rather than the signal enhancement at the sensory stages of information processing (Gould et al., 2007; Zizlsperger et al., 2012). Such an uncertainty reduction by pre-cuing has been supported by simulating psychophysical and electrophysiological data (Feldman and Friston, 2010). To address this concern of the conventional pre-cuing paradigms, we aimed to distinguish attentional sensory information processing enhancement from the effect of uncertainty reduction. Thus, we excluded a possible confound of the reduction of uncertainty in our task by using a four-alternative-forced-choice paradigm.

Attention allocation can be investigated in different behavioral contexts using different kinds of probe stimuli. One framework to study attentional effects is visual motion task, because of its particular importance for human survival. There have been some studies examining the attentional influence on linear motion discrimination (Bosworth et al., 2012; Bulakowski et al., 2007; Liu et al., 2006; Verghese et al., 2013). For example, Verghese et al. showed that attention decreases the motion discrimination threshold, when the location of the target is cued. In contrast to our knowledge about attentional influence on linear motion perception, very little is known about the effect of attention on more complex motions (such as optic flow patterns) perception. Moving through the environment, our visual system exposed to complex motion information about our surroundings, known as optic flow. Optic flow patterns are important for navigation as well as guiding actions. In one of the studies about the effect of attention on optic flow patterns, Gray used an adaptation paradigm with expanding and contracting stimuli. He showed that motion detection time is significantly shorter in high attention condition than in the poor attention condition. Thus, he concluded that attention does modulate the processing of optic flow patterns such as expansion or contraction (Gray, 2000). Spiral motions are a subset of the class of optic flow 
patterns. Spiral patterns are parameterized with the angle of their local speed to their radii, as spiral direction (Graziano et al., 1994). This parameterization of spiral patterns allows to span a circular 'spiral space' and to alter gradually spiral motion direction. Thus, spiral motion patterns are well-suited stimuli to assess attentional effect on the discrimination of optic flow patterns.

In this study, we aimed to investigate the effect of graded attention on discrimination of the spiral motion patterns in healthy human subjects. We compared the effects of attention on the spiral discrimination threshold in two experiments with four attention conditions using a pre-cueing paradigm. In both experiments, we adjusted the attentional condition by pre-cueing the target at three levels of validity. In one experiment, we additionally used a post-cue to ensure that subjects know about the target location, even when the target was invalidly pre-cued. In both experiments, the stimuli were masked to suppress any ongoing processing, since motion information and the influence of attention on them can be present even long after the stimuli disappeared (Carrasco, 2011; Karni and Sagi, 1991). The stimulus presentation was aimed to be as brief as possible. The brief stimulus presentation minimized the benefit of serial strategies and increased the difficulty of the task. We measured the performance of the subject by determining their discrimination threshold. We used adaptive testing with staircases to collect most of the data at the slope of the psychometric function.

\section{MATERIALS AND METHOD}

\section{Subjects}

We investigated the role of attention on the discrimination of spiral motion patterns by human subjects. 15 volunteers (7 females), including one of the authors (SEF) took part in two experiments. BUG, KAD, MAF, MAG, RAE, RET, SEP, SUR (2 females) participated in the first experiment and ANH, BUG, CLV, JAW, JOM, JOV, KAD, MAF, MAG, RET, SEP, SEF, TEF (8 females) participated in the second one. Four subjects were common to both experimental groups. Three subjects (KAD, JOM, RET) were excluded from the analysis. All the participants had normal or corrected to normal vision. They all, except for the author, were naive to the aim of the study and were given monetary reward for participating in the study. All participants gave written informed consent before the experiments. 


\section{Experimental setup}

Subjects were seated in a dimly lit room in front of a computer monitor with a viewing distance of $57 \mathrm{~cm}$. To maintain the subject's head position, a chin rest was used. The stimuli were displayed on a 22" widescreen TFT monitor (Syncmaster 2233RZ, Samsung Electronics, Seoul, South Korea) with a resolution of $1680 \mathrm{x}$ 1050 pixels, a refresh rate of $120 \mathrm{~Hz}$, and a spatial resolution of 46 pixels per degree of visual angle. The responses were collected by a Logitech precision gamepad (Logitech International S.A., Switzerland). Custom-made software (MWork version 0.5) running on an Apple Macintosh computer was used to present the stimuli, control the experiments and record the responses.

\section{Eye tracking}

Subjects were required to foveate a central fixation point for the whole duration of each trial and the fixation was monitored. If the subject failed to maintain his/her gaze within a central fixation window with a diameter of three degrees, the trial was aborted and repeated at a later point during the session. Eye position data was collected using an EyeLink binocular eye tracker (EyeLink 1000, SR Research Ltd., Canada). The sampling frequency was $500 \mathrm{~Hz}$.

\section{Stimuli}

The visual stimuli consisted of bright random dot patterns (RDPs) with a luminance of $72 \mathrm{~cd} / /$, displayed on a dark background with a luminance of $0.3 \mathrm{~cd} / \mathrm{l}$. The RDPs had a density of 10 dots per square degree. Each dot was a circle with 0.02 degree diameter. Three different RDPs were used as target, distracter and mask stimulus respectively.

The target stimulus contained dots moving coherently along a spiral trajectory described by the following equations:

$$
\begin{aligned}
& d r / d t=v \cos \phi \\
& d \theta / d t=\frac{v}{r} \sin \phi
\end{aligned}
$$

Where $r$ and $\theta$ are polar coordinates of each random dot and $d r / d t$ and $d \theta / d t$ are its radial and angular velocities respectively. Spiral parameters of $v$ and $\phi$ are speed and direction of the spiral motion. The local speed of every dot has a gradient linearly related to its distance from the center of the spiral trajectory ( $v=\alpha r$ ). In a given speed, spiral motions can be uniquely described by the angle of motion trajectory relative to the radii, where $\phi=0^{\circ}$ is pure expansion $(\mathrm{E}), \phi=90^{\circ}$ is 
pure clockwise rotation (CW), $\phi=180^{\circ}$ is pure contraction (C) and $\phi=270^{\circ}$ is pure counterclockwise rotation (CCW). The schematic of the spiral space is plotted in Fig.1.B.

The target stimulus was expanding spiral RDPs. The spiral RDPs were varied in angle of motion trajectory $(\phi)$ within the range of $\phi=-75^{\circ}$ to $\phi=75^{\circ}$, where RDPs with positive angle were moving CW and RDPs with negative angle were moving CCW. The speed of the RDPs had a linear gradient with $\alpha=8^{\circ} / \mathrm{s}$ and the average speed of $10^{\circ} / \mathrm{s}$.

The target stimulus was displayed in a circular aperture with a diameter of $5^{\circ}$ centered at $5^{\circ}$ left or right to the central fixation point along the horizontal midline.

Both distracter and mask stimuli were RDPs moving incoherently with random directions between 0 and 360 in spiral trajectory with the same speed and size as the target stimulus. These stimuli were displayed at the same eccentricity as the target along the horizontal midline.

To direct a subject's attention, a central cue was displayed and covered the fixation spot. The cue was a red equilateral triangle of the $0.5^{\circ}$ side's length and luminance of $13.5 \mathrm{~cd} / /$ pointing randomly to either left or right indicating the upcoming target location.

\section{Paradigm}

We had two experiments, the first one was pre-cued-only and the second one was pre\&post-cued, where subjects had to discriminate spiral motion patterns in 4AFC paradigm. Subjects were asked to report the location (left or right) as well as the rotation component (the whether the target contained clockwise or counterclockwise motion) of the target stimulus by pressing one out of four joystick buttons. We systematically varied the direction of spiral motion $\phi$ to determine the direction discrimination threshold using adaptive testing paradigm. In both experiments, we used pre-cueing paradigm to modify attention allocation by changing the cue validity at three levels of $100 \%, 75 \%$ and $50 \%$. The cue validity is a parameter of the experiment that could be associated with attention, since the subjects can direct their attention to optimize their performance using this cue. 
A

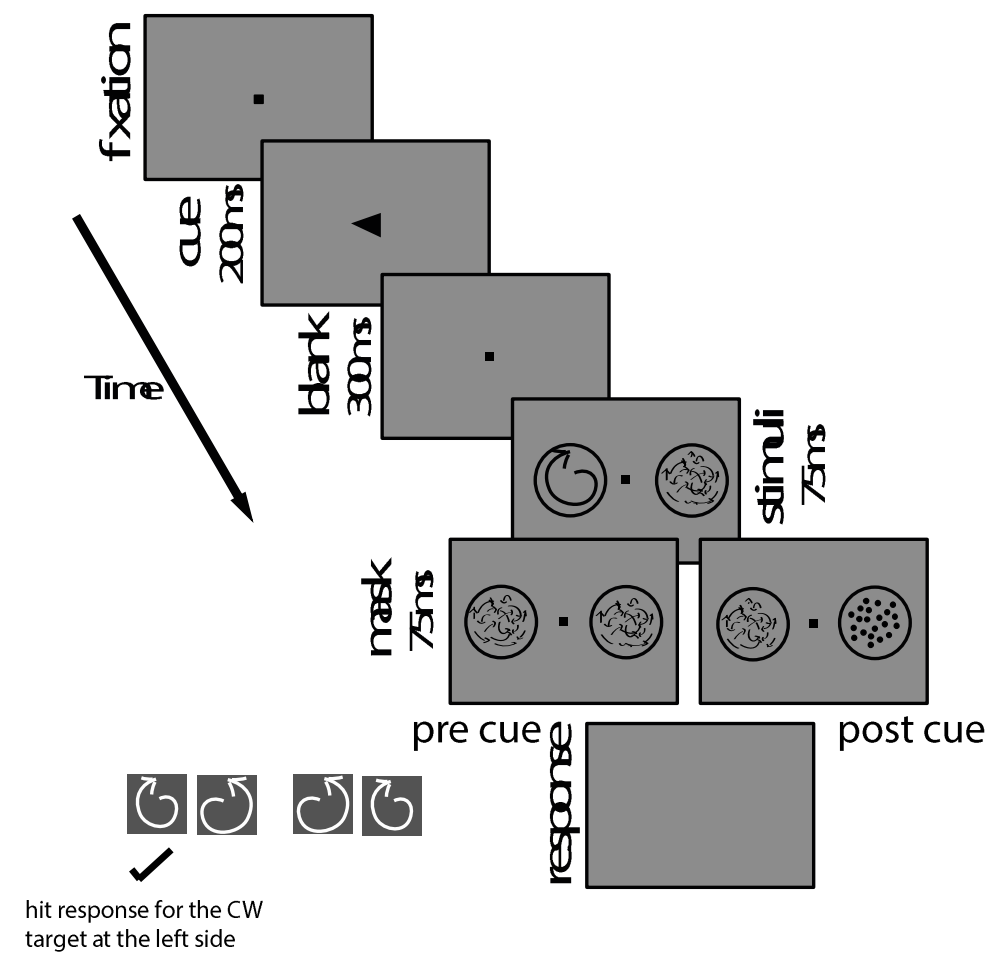

B

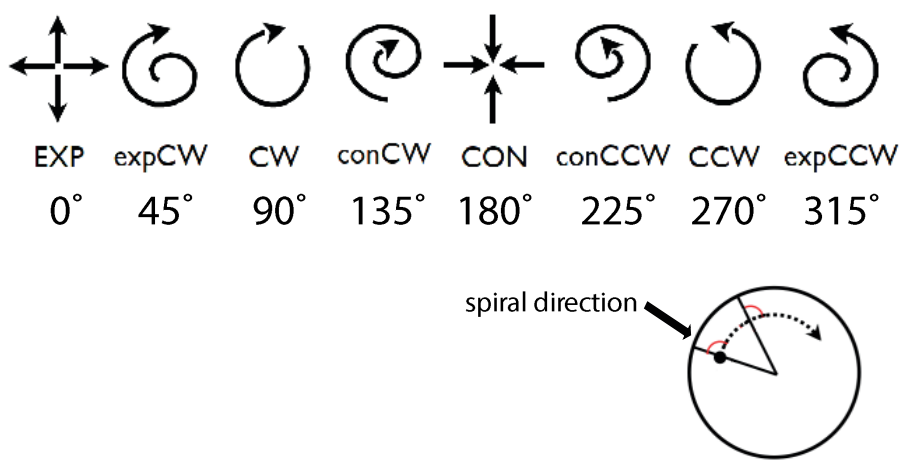

\section{Fig.1. Experimental paradigm and stimuli}

(A) Every trial started with the appearance of a fixation spot. Then the central triangle cue displayed for $200 \mathrm{~ms}$ pointing randomly to either left or right visual field, indicating the target location and followed by blank screen for $300 \mathrm{~ms}$. Then two circular apertures with 5-degree diameter filled with random dot patterns (RDPs) were displayed for $75 \mathrm{~ms}$. The target patch contained spiral moving RDPs and the distracter one contained spiral noise as randomly moving RDPs with same gradient speed as the spiral stimulus. Then, target was masked by spiral noise. In pre-cued experiment, distracter was also masked by spiral noise, whereas in post-cue experiment it mask by static dots. Subjects had to report whether the target spiral stimulus was moving $\mathrm{CW}$ or CWW. 
(B) Spiral RDPs can be defined uniquely with the one direction as the angle of their motion trajectory relative to the radii. There, $\varphi=0^{\circ}$ is pure expansion (EXP), $0^{\circ}<\varphi<90^{\circ}$ corresponds to expanding clockwise spirals, $\varphi=90^{\circ}$ is pure clockwise rotation (CW), $90^{\circ}<\varphi<180^{\circ}$ defines contracting clockwise spirals RDPs, $\varphi=180^{\circ}$ is pure contraction (CON), $180^{\circ}<\varphi<270^{\circ}$ determines contracting counterclockwise spirals RDPs, $\varphi=270^{\circ}$ is pure counterclockwise rotation (CCW) and finally $270^{\circ}<\varphi<360^{\circ}$ defines expanding counterclockwise spiral RDPs.

\section{First experiment: pre-cued-only}

In the first experiment (pre-cued-only), participants were instructed to fixate on the central fixation point during each trial and report the direction of the target spiral motion at the end of each trial. Subjects had to first fixate on the central fixation point on the computer screen to start each trial. Then, by pressing one particular joystick gamepad button, the trial started. Every trial started with the appearance of the central cue for $200 \mathrm{~ms}$, pointing to the right or to the left. Then $500 \mathrm{~ms}$ after the cue onset two stimuli, one target and one distracter, were displayed for $75 \mathrm{~ms}$. Then, the random moving RDPs mask followed both the target and distracter for another $75 \mathrm{~ms}$. The paradigm of the pre-cued experiment is illustrated in Fig.1.A.

\section{Second experiment: pre\&post-cued}

The second experiment (pre\&post-cued) had the same trial design as the first one with the difference that a static mask was following the distracter for $75 \mathrm{~ms}$ (Fig.1.A).

The first experiment (pre-cued-only), had only a pre-stimulus cue but in the second experiment (pre\&post-cued) the static mask could be used as a post-cue indicating the position of the target. In neither of the experiments, subjects were provided by any feedback about their performance during or after the experiment. The experiments were done after the training phase. The experiments were conducted in three blocks; each corresponded to one cue validity level and contained 320 trials. The order of performing these three blocks was assigned randomly.

In both experiments, we tried to find the stimulus duration, which minimize the benefit of shifting attention from one side to the other. Hence, we ran a pilot measurement with six subjects on $100 \%$ cue validity condition using the first experiment (pre-cued-only) trial design. We saw if the stimulus duration decreases 
gradually, at about $80 \mathrm{~ms}$ the subject's threshold will increase, and subject will fail to do the task if the stimulus exposure time is less than 60ms (Sup.Fig.1). Thus, we set the target stimulus duration to a brief duration of $75 \mathrm{~ms}$. At this duration, subject cannot do the task on both sides on a serial fashion, as $s /$ he has only less than $40 \mathrm{~ms}$ to do the task on each side. It means if subject wants to randomly preallocate his/her attention to one side and in case of misallocation of attention, shift his/her attention to the other side to do the task, s/he will run out of the time and cannot accomplish the trial successfully. Moreover, converging evidences from several psychophysics as well as monkey electrophysiological studies suggest the optimum stimulus exposure of about 100 ms or less (Jans et al., 2010), which is consistent with our stimulus duration.

In order to monitor participants responses accurately, four-alternative-forcedchoice responding was used. Subjects had to press the upper buttons on the gamepad to answer for clockwise RDPs and lower buttons for counterclockwise one. Moreover, they had to use the left buttons for the left-sided target and right buttons for the right-sided one. Using four-forced-choice responding allows us to distinguish between the two different kinds of failed answers, the wrong discrimination of the spiral RDP type (when subject press the wrong button but at target side) or the wrong detection of the target (when subject press any button at the distracter side). This way, we could exclude the uncertain answers (answers to the distracter) as a possible confound of the uncertainty reduction caused by precuing the target side in our task.

We altered the cue validity in three levels to modify attention allocation. In the $100 \%$ cue validity level, the cue always signaled the location of the target stimulus correctly. We called this the $100 \%$ attention condition. In the $75 \%$ cue validity, in $75 \%$ of trials, the target stimulus appeared at the cued location $(75 \%$ attention condition). In the remaining $25 \%$ of trials, the target happened at un-cued side (25\% attention condition). Finally, in the $50 \%$ cue validity, cue has no information about the target location. As both sides were equally to be the target, attention was considered to be at both target and distracter equally. Thus, the target (at both cued and un-cued locations) was considered to have $50 \%$ attention. Therefore, in both experiments we had four attention conditions of: $100 \%, 75 \%$, $50 \%$ and $25 \%$. 


\section{Adaptive testing}

We used an adaptive testing strategy by using four interleaved staircases. The upper staircases started from clockwise spiral direction and two consecutive clockwise answers decreases the (spiral direction) of the next stimulus one step-size; and a counterclockwise answer increases the of the next stimulus one step-size. Thus, the sampling range targets toward $70.71 \%$ frequency of $\mathrm{CW}$ answers. Similarly, the lower staircases started from counterclockwise spiral direction, again with 1-2 rule, where is decreased after each clockwise response and increased after two consecutive counterclockwise answers. Using such staircases provides more samples away from the asymptotes and closer to the $29.29 \%$ and $70.71 \%$ parts of the psychometric function, which eventually results to more reliable estimate of the discrimination threshold. The staircases were randomly interleaved to reduce the possibility of the anticipation of the next presented stimulus by the subject. This anticipation could cause a change in subjects responding strategy such that the actual decimation threshold cannot be measured. Fig. 3 displayed the staircase data of one sample subject.

\section{Training}

In first training session after explaining the experiments as well as the visual stimuli to the subjects, they performed 4-5 blocks, each with 320 trials, for $100 \%$ cue validity and one block of 320 trials on $50 \%$ cue validity. Presentation duration of target stimulus was gradually decreased from $500 \mathrm{~ms}$ to $75 \mathrm{~ms}$ after about each 100 trials during the first two blocks. Then subjects performed two more blocks with the stimulus duration of $75 \mathrm{~ms}$. The second training session resumed with 4-5 blocks, each containing 320 trials. Similar to the first training session, the target stimulus duration reduced gradually to $75 \mathrm{~ms}$ but with the cue validity of $50 \%$. Then subjects performed two more blocks with the stimulus duration of $75 \mathrm{~ms}$ and cue validity of $50 \%$. They also performed one more block with the cue validity of $75 \%$ and stimulus duration of $75 \mathrm{~ms}$. If the subject did not reach the plateau of his /her discrimination threshold in the second training session for $50 \%$ cue validity, then he/she would be trained one or even two more sessions until his/her discrimination threshold reached its plateau. All together, each subject performed more than 2000 training trials. We excluded participants, whose one of their thresholds for left and right targets after the training was more than $18^{\circ}$ in $100 \%$ attention condition. The $18^{\circ}$ exclusion criterion was more than the 84 th percentile of the subject's 
threshold distribution in $100 \%$ attention condition. More detailed explained in the result session.

\section{Data Analysis}

All related analysis for computing the discrimination threshold was done using "Palamedes: Matlab routines for analyzing psychophysical data" (Coutanche, 2013). The final statistical analysis was done using MATLAB (version 2011b, The MathWorks, Inc., USA).

Based on the subjects' responses, their psychometric function was estimated. The psychometric function was computed by fitting the following logistic equation to the response frequency of the clockwise answers to all presented spiral directions at the target (Kingdom and Prins, 2010) . In this equation, " $\mathrm{s}$ " is the direction of spiral motion and " $F(s)$ " is the response frequency for the clockwise rotation at the direction of "s". "a" and "b" are fitted parameters.

$$
F(s)=\frac{1}{1+e^{(-b \times(s-a))}}
$$

Then, the discrimination threshold " $T$ " was defined as the distance between $\mathrm{F}(\mathrm{s})=$ 0.5 (PSE) and $F(s)=0.84$.

Moreover, the fitting algorithm computed the maximum likelihood estimation using parametric bootstrapping and provided it as a goodness of fit (GoF) index. Considering 'Palamedes' developers' recommendation, all fits with the GoF of less than 0.05 cannot be used. In addition, we also computed the sum-of-squarederrors as well as the coefficient of determination (R-squared) as additional indices for the goodness of the fits.

The confidence intervals of the threshold and other estimated parameters were computed using parametric bootstrapping with 400 repetition (Kingdom and Prins, 2010). Confidence intervals of the determined thresholds are informative about the reliability of their determination. Thus, if the percentage of the confidence interval to the determined threshold was more than $100 \%$, we excluded that threshold as a noisy measurement from the further analysis. Furthermore, we used the inverse of the confidence intervals as a weight vector in a weighted linear correlation analysis to reduce the variability induced by measurement noise. More detailed explained in the result session.

Beside the discrimination threshold, the point of subjective equality (PSE) of each psychometric function was determined. PSE is the direction in which the subject's response is $50 \%$ counterclockwise. It specifies the difference between the PSE 
and point of physical equality (PPE) in discrimination counterclockwise moving spirals. The detailed extracted parameters (threshold $\pm S D, P S E \pm S D$ and GoFs) for each subject were summarized in their corresponding supplementary table.

For each experiment, the discrimination thresholds were determined for both left and right spiral targets, at each cue validity level of $100 \%, 75 \%$ and $50 \%$. It yielded to totally 10 determined thresholds (Right Targets: $100 \%$ cued valid, $75 \%$ cued valid, $75 \%$ invalidly cued, $50 \%$ cued valid, 50\% cued invalid \& Left targets: 100\% cued valid, $75 \%$ cued valid, $75 \%$ invalidly cued, $50 \%$ cued valid, $50 \%$ cued invalid) for each experiment. As there was no significant difference between the determined threshold for the 50\% cued valid and 50\% cued invalid targets, their trials were pooled together to determine the threshold for $50 \%$ cued condition. We considered $100 \%$ cued valid as $100 \%$ attention, $75 \%$ cued valid as $75 \%$ attention, $75 \%$ cued invalid as $25 \%$ attention and $50 \%$ cued as $50 \%$ attention condition. Hence, there were four determined discrimination thresholds for each attention condition of $100 \%, 75 \%, 50 \%$ and $25 \%$ for both left and right stimuli. Fig. 2 depicts sample subject psychometric functions with the determined thresholds. These determined thresholds were then used for the statistical analysis described in the result section.

In order to determine the psychometric functions, frequency of CW answers to each sampled spiral direction was computed from the answers to the target side, and fitted to the logistic equation. The trials with the answers to the distracter side, i.e. with the detection failure, were excluded from the fitting and data analysis. By excluding them, we discarded the influence of uncertain answers, in order to discriminate the effects of sensory enhancement form the uncertainty reduction in our experiments.

\section{RESULTS}

We investigated the effect of attention on discriminating spiral motion patterns by varying the pre-cue validity in order to modify attention allocation to the target stimulus at four levels. Eight subjects participated in the first experiment (pre-cuedonly). Twelve subjects participated in the second experiment (pre\&post-cued).

As the first step of the analysis, we applied an inclusion criterion to exclude subjects with outlier threshold at $100 \%$ attention condition from the further analysis. The outlier thresholds could skew the mean, inflate the standard 
deviation and ultimately reduce power of the statistical tests. We pooled the determined thresholds from left and right stimuli to determine the cut-off criterion after testing if obtained thresholds from left and right targets in 100\% attention condition were following the same distribution. We saw no significant difference between them using the paired-Kolmogorov-Smirnov test (in pre-cued experiment: $p=0.92$, ks2stat $=0.25$, in post-cued experiment: $p=0.88$, ks2stat=0.22). At the second level, we tested whether two distribution have the same median using Wilcoxon signed rank test, and saw no difference (exp. 1: $p=0.95$, exp.2: $\mathrm{p}=0.74$ ). Thus, we included all thresholds and then computed their 84 th percentile (corresponding to one standard deviation in normal distribution) in each experiment separately. The histogram of the discrimination thresholds in $100 \%$ attention condition is plotted in Sup.Fig.2 and Sup.Fig.3 for experiments one and two respectively. We set the inclusion criterion for the discrimination thresholds to 18 degrees, which is bigger than the 84th percentile in both experiments. Therefore, all the subjects with the one threshold bigger than 18 degrees in the $100 \%$ attention condition were excluded from the main analysis. Two subjects (KAD, RET) could not pass our inclusion criterion. Besides, one more subject (JOM) was removed from the study because he could not pass the training.

In the second step, we tested whether the determined thresholds in 50\% attention condition are different in valid cued vs. invalid cued for both experiments. We saw no significant difference between these conditions (in pre-cued experiment: rmANOVA, $F=2.08, p=0.21$, in post-cued experiment: $\operatorname{rmANOVA}, F=0.002$, $p=0.99$ ). Therefore, we pooled the trials across valid and invalid cued conditions to determine one threshold for $50 \%$ attention condition for target at each side. Hence, we had $2 \times 4$ full factorial design matrix (side = left, right), attention= $(25 \%, 50 \%$, $75 \%, 100 \%$ ) of the threshold values in each experiment. Thus, we performed repeated measured ANOVA for statistical analysis. Furthermore, we considered the threshold values determined from non-acceptable fits (GoF < 0.05) or thresholds with more than $100 \%$ error (100xconfidence-interval/threshold) as missing values for the statistical analysis.

\section{First experiment: pre-cued-only}

We aimed to investigate the effect of graded allocation of spatial attention in discriminating spiral patterns. Thus, we compared the discrimination threshold of spiral motion pattern across four attention conditions to see whether the 
discrimination threshold is changing by allocating more attention to the target. The discrimination thresholds of six subjects (for both left and right spiral stimuli) in the four attention conditions are shown in Fig.4.A. The large number of uncertain answers to the distracter in $25 \%$ condition yielded to few numbers of data points ( $~ 5$ data points) for the fitting. Therefore, the fits did not converge and we failed to determine threshold in $25 \%$ attention condition. The averaged discrimination thresholds were $12.2^{\circ}, 12.5^{\circ}$ and $15.9^{\circ}$ for $100 \%, 75 \%$ and $50 \%$ respectively. The median of the discrimination thresholds were lowest for the $100 \%$ condition $\left(12.1^{\circ}\right)$, higher for the $75 \%$ attention condition $\left(12.9^{\circ}\right)$ and highest for the $50 \%$ attention condition $\left(16.9^{\circ}\right)$. We compared the mean of discrimination thresholds in the four levels of attention, and saw promising effect of attention on the discrimination threshold of spiral motion ( $\mathrm{mANOVA}, \mathrm{F}=3.31, \mathrm{p}=0.11$ ). Moreover, there was no significant effect of side (rmANOVA, $F=0.84, p=0.4$ ) as well as the interaction between side and attention (rmANOVA, $F=1.52, p=0.27$ ) on the thresholds. We used the weighted general linear model to test whether the discrimination threshold changes linearly by the allocated attention across the four attention conditions. We used weighted general linear model to improve the power of our analysis by confounding the variability of the determined thresholds in each attention condition. The estimated standard deviations of the determined thresholds indicate their variability. Therefore, we used the inverses of the standard deviations as the weights for the general linear model. The weighted general linear model showed a promising negative linear correlation between attention and discriminated threshold with a $-11 \%$ threshold modulation $(p=0.13)$ when attention allocation alters from $50 \%$ to $100 \%$. This result indicates that attention decreases the discrimination thresholds. The resultant line of weighted general linear model is shown with the solid line in the Fig.4.A. The weighted mean of the discrimination thresholds were $12^{\circ}, 12.4^{\circ}$ and $14.9^{\circ}$ for $100 \%, 75 \%$ and $50 \%$ respectively, and depict with dash-line in the Fig.4.A.

Since we were interested on the effect of attention on the mean of the threshold, we normalized the determined thresholds to the threshold in 100\% attention condition to compensate for the inter-subject variability. The normalized discrimination thresholds were plotted in Fig.4.B. We observed a promising modulation of attention ( $r$ ANOVA, $F=2.51, p=0.16$ ), no significant side effect (rmANOVA, $F=0.46, p=0.53$ ) and no significant interaction between side and 
attention ( $r m A N O V A, F=0.8, p=0.46$ ) on the normalized discrimination thresholds. The weighted general linear model revealed $-13 \%$ threshold modulation $(p=0.02)$ by increasing the amount of allocated attention from $50 \%$ to $100 \%$. Solid line in Fig.4.B represents the resultant line of weighted general linear, and the dash-line depicts the weighted mean.

It the pre-cued-only experiment we had a large amount of answers to the distracter (ignored trials), which was strongest at the $25 \%$ attention condition (with about $80 \%$ of ignored trials in $25 \%$ attention, and $40 \%$ ignored trials in $50 \%$ attention) as shown in the Fig.4.C. Consequently, we lost a very large proportion of the trials, which caused poor fitting with many unacceptable GoFs or unreliable determined thresholds, which ultimately yields many missing values for the thresholds in $50 \%$. Moreover, the small number of included trails induced variability to the obtained thresholds, which might attenuate the statistical power of the rmANOVA.

\section{Second experiment: pre\&post-cued}

We introduced a post cue to the second experiment to minimize the number of ignored trials observed in the first experiment. Fig.5.C illustrated the percentage of wrong side answers in all attention conditions and demonstrated the success of the post-cue in reducing the answers to the distracter. Although the post-cue resolved the problem of answering to the wrong side, it might affect the subjects' answering strategy by changing the 4AFC to a 2AFC. Therefore, we tested the difference between the obtained thresholds in the $100 \%, 75 \%$ and $50 \%$ attention conditions across the two experiments and saw no significant difference between them ( $r m A N O V A, F=0.001, p=0.97$ ). Same as the first experiment, we plotted the determined thresholds for the four attention conditions (shown in Fig.5.A). Average discrimination thresholds were lowest for $100 \%\left(9.2^{\circ}\right)$, higher for $75 \%\left(10.7^{\circ}\right)$ and more for $50 \%\left(12.8^{\circ}\right)$ and $11^{\circ}$ for $25 \%$. The median of the discrimination thresholds were $8.3,9.8,13$ and 10 for $100 \%, 75 \%, 50 \%$ and $25 \%$ respectively. We observed a significant reduction in the discrimination thresholds with higher attention ( $r m A N O V A, F=3.74, p=0.02$ ). We did not see any significant side ( $r m A N O V A, F=0.96, p=0.35$ ) or interaction effect (rmANOVA, $F=1.36, p=0.28$ ). The weighted general linear model of the thresholds revealed a $-9 \%$ modulation of the discrimination threshold by increasing the amount of allocated attention from $50 \%$ to $100 \%(p=0.02)$. 
A

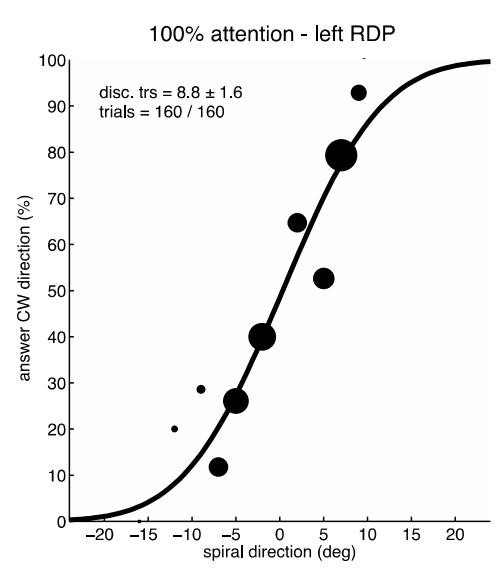

C

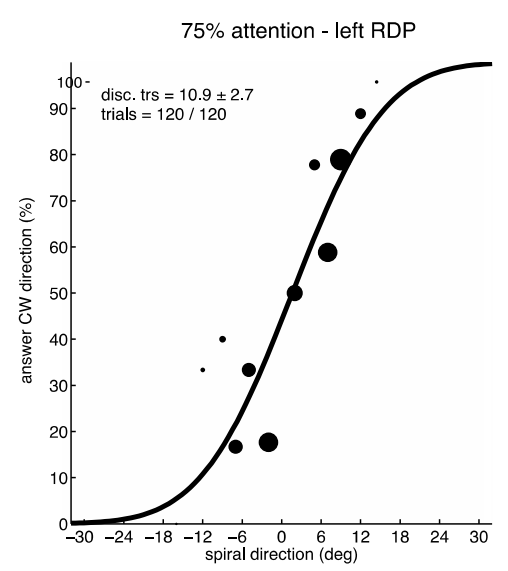

E

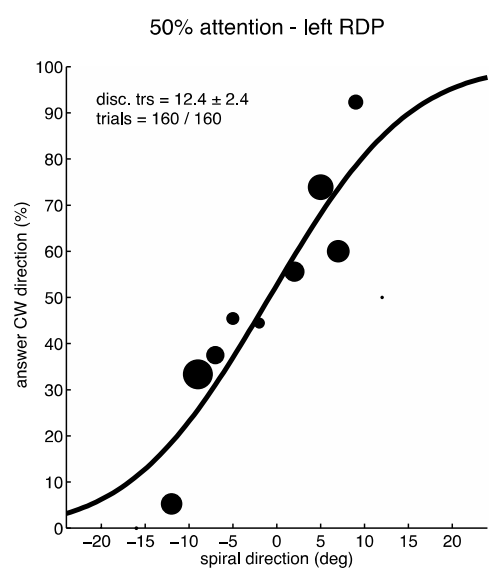

G

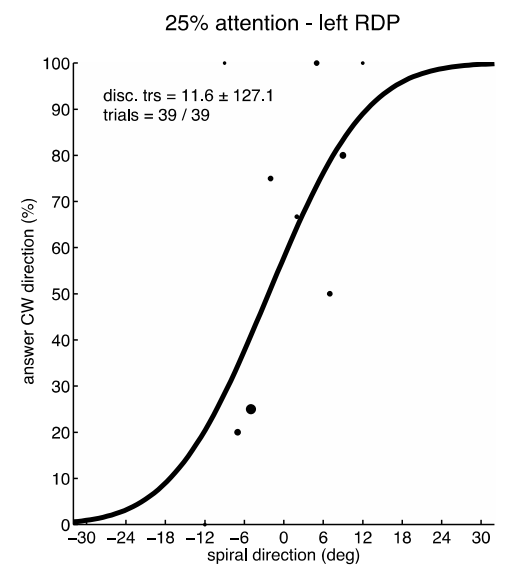

B

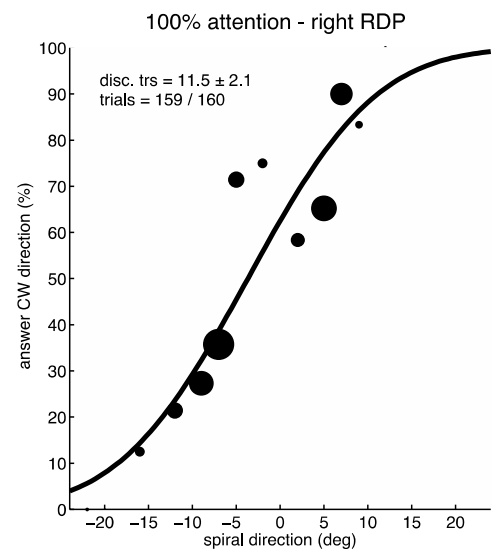

D

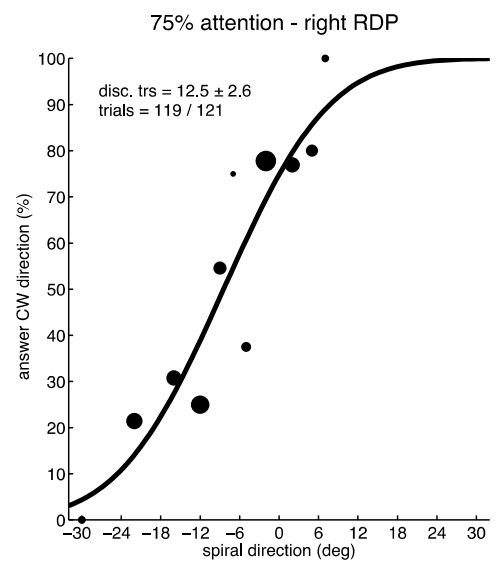

F

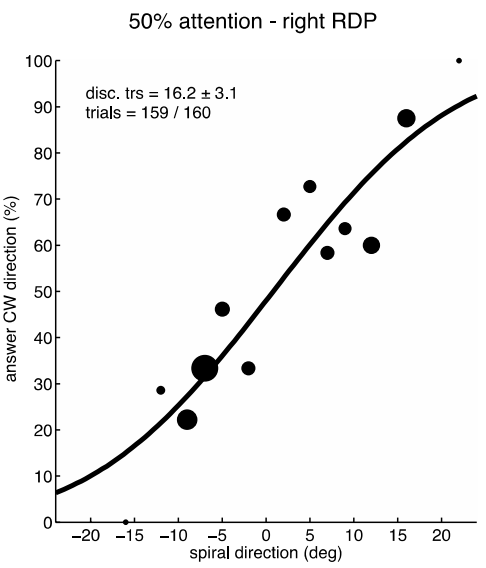

H

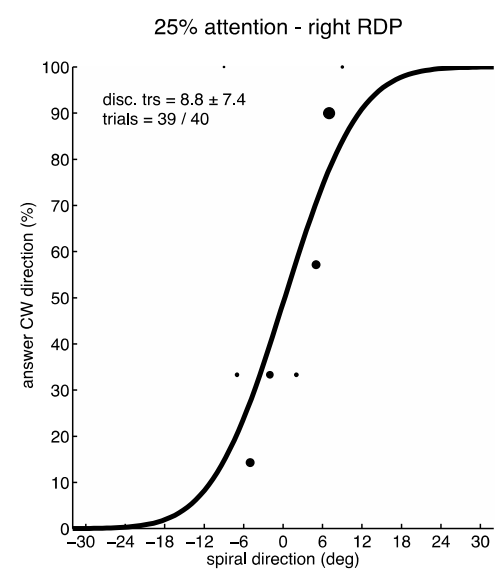




\section{Fig.2: Sample subject (anh) psychometric functions in experiment 2}

(A) Psychometric curve for the left stimulus in the $100 \%$ attention condition is illustrated as the function of the frequency of the clockwise answers ( $y$-axis) to each presented spiral direction ( $x$ axis). The repetition of each sampled spiral direction is linearly related to the diameter of its corresponding dot marker. Discrimination threshold, and number of the included trials for each fit is displayed on the figure. (B) Psychometric function for the right stimulus in the $100 \%$ attention condition (C) Psychometric function for the left stimulus in the $75 \%$ attention condition (D) Psychometric function for the right stimulus in the $75 \%$ attention condition (E) Psychometric function for the left stimulus in the 50\% attention condition (F) Psychometric function for the right stimulus in the $50 \%$ attention condition (G) Psychometric function for the left stimulus in the $25 \%$ attention condition $(\mathrm{H})$ Psychometric function for the right stimulus in the $25 \%$ attention condition.

Target stimulus on left side

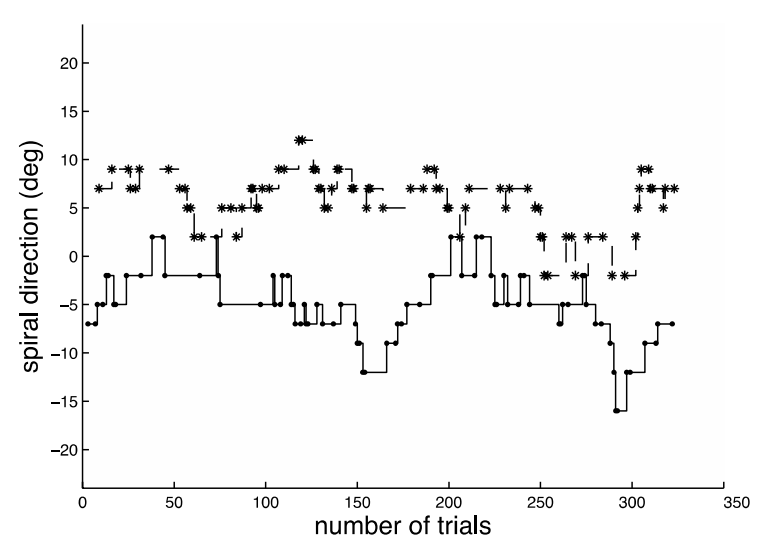

Target stimulus on right side

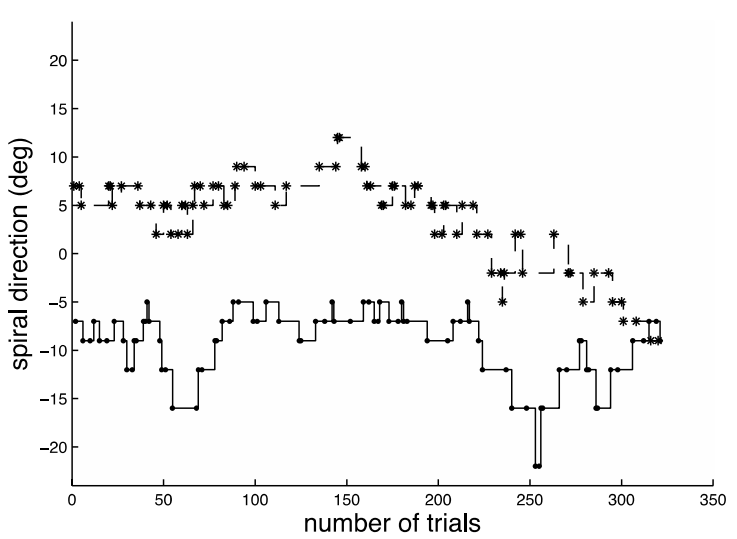

Fig.3: Sample subject (anh) staircase plot at $100 \%$ cue validity in experiment 2

There were two interleaved staircases for each stimulus at each cue validity condition. The staircases value will get one step closer to PSE in case of two consecutive answers same as the staircase direction, and will get one step away from the PSE in case of any answer at the opposite direction to the staircase. The staircase with solid-line started from clockwise spiral direction and the staircase with dash-line started sampling counterclockwise spiral RDPs.

The resultant line of weighted general linear model as well as the weighted mean of the discrimination thresholds are plotted in solid and dash lines in Fig.5.B respectively.

Moreover, the normalized discrimination thresholds in four attention conditions were depicted in Fig.5.B. The normalized thresholds decreased with attention across the four levels of attention (rmANOVA, $F=4.09, p=0.02$ ). There was no side effect ( $r$ ANOVA, $F=4.82, p=0.07$ ) and no interaction between side and attention 
( $r m A N O V A, F=0.87, p=0.45$ ) on normalized thresholds. We observed $-10 \%$ modulation of discrimination threshold by halving the amount of allocated attention using weighted general linear model $(p=0.008)$. The resultant line of weighted general linear depicts in Fig.5.B by solid line and the dash-line represents the weighted mean of the discrimination thresholds.

A

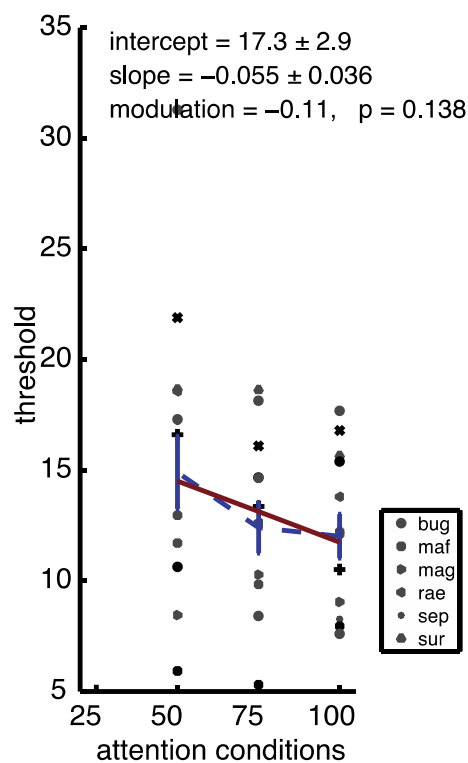

B

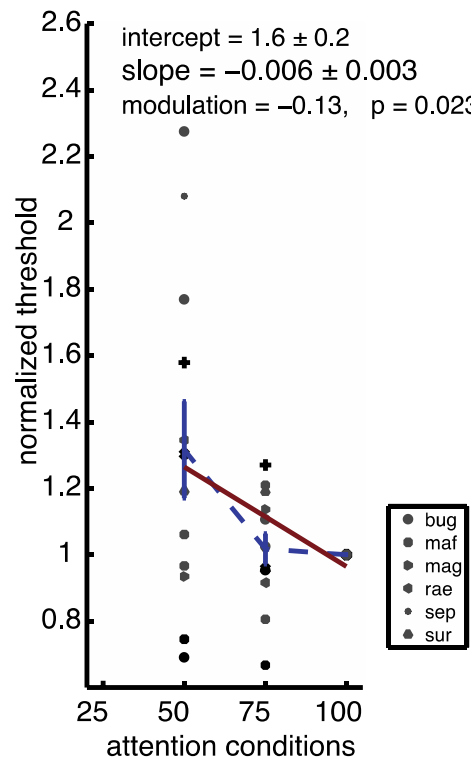

C

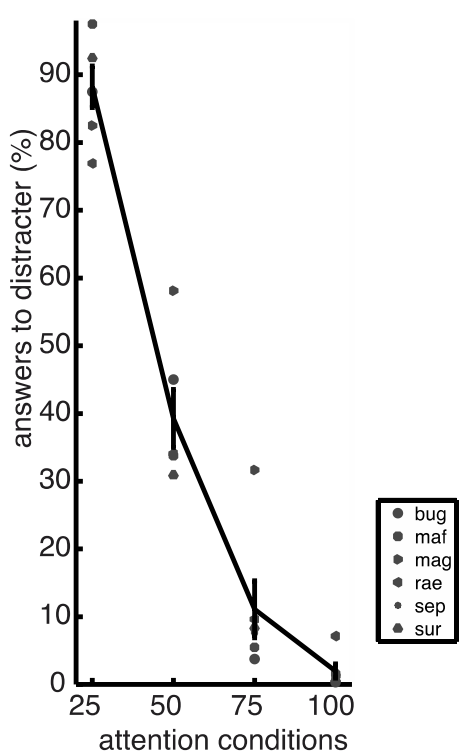

\section{Fig.4: Graded attention in discriminating spiral patterns in experiment one}

(A) $\mathrm{X}$-axis represents four attention conditions, while $\mathrm{Y}$-axis corresponds to the thresholds. The thresholds in each attention condition are plotted at each attention condition for the population of six subjects. The black solid line represents the line obtained from the weighted general linear model of the determined thresholds in three attention conditions (intercept $=17.3^{\circ} \pm 2.9^{\circ}$, slope $=$ $-0.05 \pm 0.03$ ). The general linear model showed $-11 \%$ modulation of the discrimination thresholds by halving the amount of allocated attention $(p=0.13)$. The weighted mean of the determined thresholds in three attention conditions depicts with dash line. Error bars represent the standard error of the weighted mean. The data from each individual subject, at each side is shown with the different symbol. (B) The extracted thresholds from psychometric functions in each attention conditions are normalized to the determined threshold in $100 \%$ condition. The normalized thresholds are plotted in a same way as Fig. 4A. The weighted fitted line to the thresholds had the intercept $=1.6 \pm 0.2$ and the slope $=-0.006 \pm 0.003$. The general linear model revealed $-13 \%$ modulation of the thresholds by halving the amount of allocated attention $(p=0.02)$.(C) The percentages of the answers on the wrong sides with uncertainty error are plotted in each attentional condition. The black solid line represents their mean across six subjects. Error bars represent the standard error of the mean. The data from each individual subject, at each side is shown with different symbol. 
A

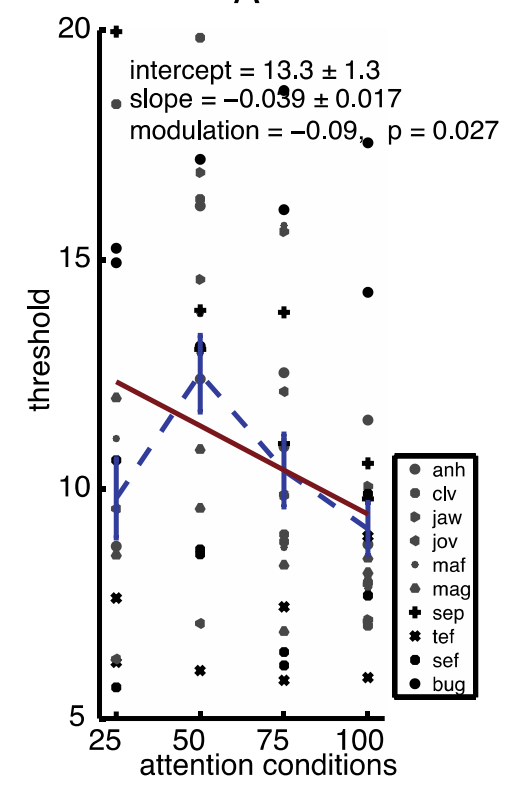

B

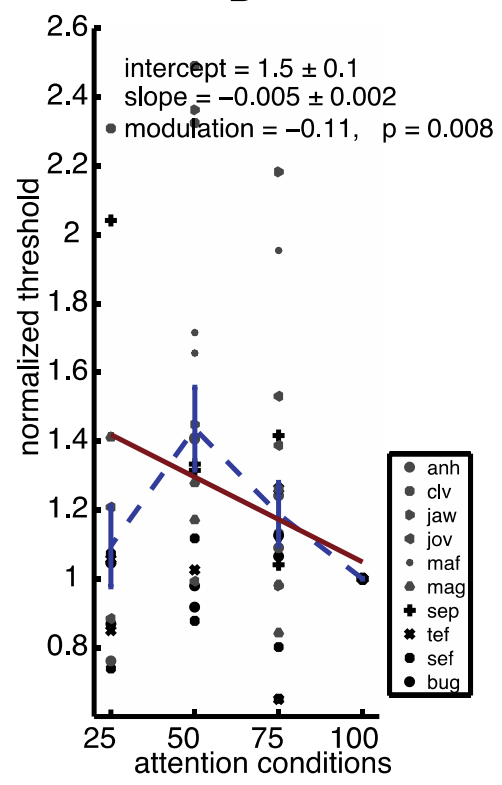

C

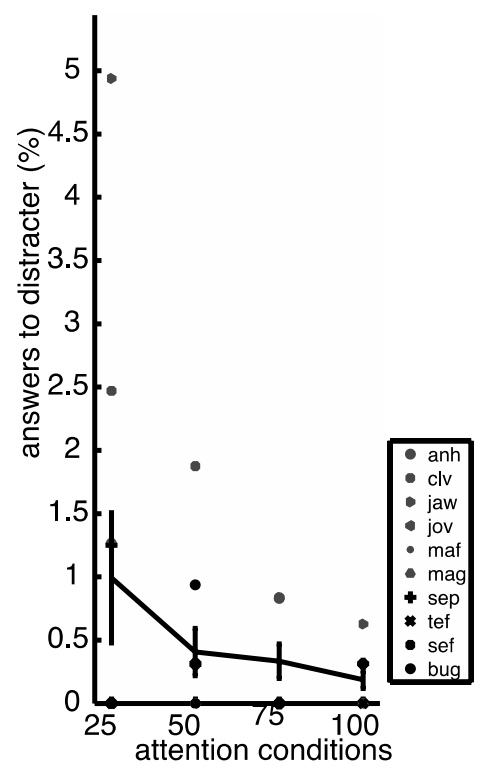

Fig.5: Graded attention in discriminating spiral patterns in experiment two

(A) The extracted thresholds from psychometric functions in each attention condition are plotted in a same way as Fig. $4 \mathrm{~A}$ across ten subjects. There was a significant difference between the threshold in the four attention conditions ( $p=0.02$ rm-ANOVA). The weighted fitted line to the thresholds had the intercept $=13.3 \pm 1.3$ and the slope $=-0.03 \pm 0.001$. The general linear model showed $-9 \%$ threshold modulation by halving the amount of allocated attention $(p=0.02)$.

(B) The extracted thresholds from psychometric functions in each attention condition are plotted with normalization in a same way as Fig. 4B. Repeated measured ANOVA revealed a significant difference between the threshold in the four attention conditions ( $p=0.02$ rm-ANOVA). The weighted fitted line to the thresholds had the intercept $=1.5 \pm 0.1$ and the slope $=-0.005 \pm 0.002$. The general linear model showed $-11 \%$ threshold modulation by halving the amount of allocated attention $(p=0.008)$.

(C) The percentage of the answers to the distorter are plotted in each attentional conditions. The black solid line represents the average of them across ten subjects. Error bars represent the standard error of the mean.

\section{DISCUSSION and CONCLUSIONS}

We investigated graded attention by testing whether we can have a graded modulation of the spiral motion discriminating thresholds across different conditions by altering the target relevance for the task. We hypothesized whether it is possible to distribute the attentional resources to the stimuli in a graded way 
based on their relevancy for the task. Thus, we used a pre-cuing paradigm where we varied the validity of the pre-cue with the aim to modify the relevance of the spiral stimulus in four levels of $25 \%, 50 \%, 75 \%$ and $100 \%$, corresponding to four attention conditions. We then tested whether the discrimination threshold of spiral motion patterns is changing across these levels of attention. And, if there was a difference across these condition, whether it was linearly correlated with the conditions.

We saw a pattern in discrimination thresholds in both experiments. In first experiment, the mean, the median and the weighted mean of the thresholds were lowest in $100 \%$ condition, higher in $75 \%$, and highest for the $50 \%$, though we failed to have any reliable value in $25 \%$ condition. Weighted general linear model demonstrates about $-10 \%$ modulation of the threshold by halving the amount of allocated attention. In the second experiment, the similar pattern stayed for the mean, the median as well as the weighted mean of the thresholds. We also observed similar modulation of discrimination threshold by attention using weighted general linear model. In $25 \%$ attention condition, the small number of data points fed to the fit increased the variability in the determined thresholds and we consequently had to replace five thresholds out of twenty with the missing values. This high variability in the determined thresholds might potentially decline the reliability of the estimated mean, the median and even the weighted mean in $25 \%$ attention condition, though the weights could somehow degrade the effect of the high variability. The determined modulation of the discrimination threshold in this study is in agreement with the general conclusion of several psychophysical studies of attentional effect on motion processing, which reported that pre-cueing a specific part of the visual field enhances the subject performance by decreasing his/her motion-discrimination threshold (e.g. Liu et al., 2006; Verghese et al., 2013).

The amount of the observed attentional modulations of the discrimination thresholds in our both experiments are quantitatively in line with the reported attentional modulation of the neuronal responses in monkeys' area MT (Cook and Maunsell, 2002; Treue and Maunsell, 1999) as well as the attentional modulation of the BOLD signal in humans' area MT and MST which was determined in the chapter two. This similarity between the behavioral and neuronal modulations 
confirms the significant role of the MT-complex as the specialized area in processing of spiral motion patterns.

Similar to our finding, it has been reported that the influence of the exogenous spatial varies with cue salience in a graded fashion (Fuller et al., 2009). In addition, the neuronal correlate of the graded attention was examined in an EEG study (Gould et al., 2011). There, it was shown that alpha oscillation over the occipital cortex gradually changes by altering cue-validity. Given together, our results were consistent with the reported attentional strategies, where the subjects allocated the greatest amount of attention to the most relevant stimulus and less to the less significant one.

In the first experiment, we saw no significant but promising difference between the mean of the discrimination thresholds in the four grades of attention allocation using rmANOVA. However, we saw a significant negative linear correlation between the attention allocation and the discrimination threshold using weighted general linear model. The lack of the significant effect of graded attention on the mean of the thresholds seemed to be because of the attenuated power of the rmANOVA test caused by many missing values as well as unreliable thresholds in $25 \%$ and $50 \%$ attention conditions. Indeed, enormous trials that were affected by uncertainty were unusable for the analysis. This consequently decreased the goodness of the fits and yielded many missing or unreliable discrimination thresholds. Hence, we tried to address this issue by removing the mask at the distracter, which served a post-cue. Unfortunately removing the mask did not resolve the problem completely, though it reduced the number of wrong side answers (Sup.Fig.4). We then introduced a stronger post-cue by masking the distracter with static dots. This design, which is the second experiment, could successfully resolve the problem of the wrong side answers. However, it is prone to the change of the answering strategy from $4 A F C$ to $2 A F C$ by subjects. Since the post cue happened after the stimuli presentation, it might not affect the sensory processing of the stimulus, however, it might influence the subject strategy in answering by decreasing his/her decision uncertainty, as it removed the uncertainty about the target side. We addressed this criticism by comparing the thresholds' distribution between pre-cued and post-cued experiments and saw no difference between them. Therefore, we could conclude that the observed effect 
was mostly related to the stimulus-processing enhancement rather than uncertainty reduction at the decision-making level.

The obtained results from the first experiment suffer from the vast variability induced by the fitting. One way to reduce such a noise is discarding the data with unreliable fits or determined thresholds from the analysis by defining a proper inclusion criterion. Besides, giving a weight to the determined threshold based on their reliability (e.g. their confidence interval) seemed as a proper approach to overcome induced variability in the data. Consequently, a proper statistical test could be modified to capture the weights, which is planned as a follow up for this study.

It has been shown (Bosworth et al., 2012) that the effects of spatial attention on motion discrimination are greater in the left visual field. We also check this by performing repeated measure ANOVA and saw no significant interaction between the side and attention, in disagreement with the (Bosworth et al., 2012) result.

We also checked whether the discrimination thresholds are systematically different for the right versus left targets. Using the repeated measured ANOVA, we saw no significant side effect on the thresholds in both experiments. Moreover, Sup.Fig.5 and Sup.Fig.6 illustrated the scatter plot of the determined discrimination thresholds of the left and right stimuli across all attention conditions for the experiment one and two respectively. We used general linear model with the obtained thresholds form the right stimulus as its predictors and the obtained thresholds from the left stimulus as the observations. The resultant line of the general linear model had no significant slope than one in the first experiment. It had a significant slope but very close to one $(0.9 \pm 0.15)$ in the experiment two. Thus, considering the results from both general linear model as well as the repeated measured ANOVA, we could conclude that there was no systematic effect of the side on discrimination threshold.

We also assessed the distribution of the determined PSEs to check whether they do endure any bias toward clockwise or counterclockwise spirals. The obtained PSEs pooled across all attention conditions from the first experiment had the mean of -1.9 and the median of $-1.6^{\circ}$. The histogram of the all included PSEs is plotted in Sup.Fig.7, which showed no significant trend toward any spiral direction (ttest: $p=0.06$, Wilcoxon signed rank test: $p=0.09$ ). In the second experiment, PSEs distribution had the mean of $0.53^{\circ}$ and the median of $0.29^{\circ}$. Its histogram is 
plotted in Sup.Fig.8 without any significant trend toward any spiral direction (ttest: $p=0.22$, Wilcoxon signed rank test: $p=0.3$ ). The results indicate that point of subjective equality is the same as the point of physical equality for spiral motion patterns.

In summary, we found an indication for the possibility of allocating the spatial attention recourses in a graded fashion to the more behaviorally relevant stimuli. 


\section{BIBLIOGRAPHY}

Awh, E., Pashler, H., 2000. Evidence for split attentional foci. Journal of Experimental Psychology: Human Perception and Performance 26, 834-846.

Bashinski, H.S., Bacharach, V.R., 1980. Enhancement of perceptual sensitivity as the result of selectively attending to spatial locations. Perception \& Psychophysics 28, 241 248.

Bosworth, R.G., Petrich, J.A., Dobkins, K.R., 2012. Effects of spatial attention on motion discrimination are greater in the left than right visual field. Vision Research 52, 11-19.

Braun, J., Sagi, D., 1990. Vision outside the focus of attention. Perception \& Psychophysics 48, 45-58.

Brefczynski, J.A., De Yoe, E.A., 1999. A physiological correlate of the 'spotlight' of attention. Nature Neuroscience 2, 370-374.

Bulakowski, P.F., Bressler, D.W., Whitney, D., 2007. Shared attentional resources for global and local motion processing. Journal of Vision 7, article 10.

Carrasco, M., 2011. Visual attention: The past 25 years. Vision Research 51, 1484-1525.

Carrasco, M., McElree, B., 2001. Covert attention accelerates the rate of visual information processing. Proceedings of the National Academy of Sciences 98, 5363-5367.

Cook, E.P., Maunsell, J.H.R., 2002. Attentional modulation of behavioral performance and neuronal responses in middle temporal and ventral intraparietal areas of macaque monkey. Journal of Neuroscience 22, 1994-2004

Coutanche, M.N., 2013. Distinguishing multi-voxel patterns and mean activation: why, how, and what does it tell us? Cogn Affect Behav Neurosci 13, 667-673.

Dobkins, K.R., Bosworth, R.G., 2001. Effects of set-size and selective spatial attention on motion processing. Vision Research 41, 1501-1517.

Doricchi, F., Macci, E., Silvetti, M., Macaluso, E., 2010. Neural correlates of the spatial and expectancy components of endogenous and stimulus-driven orienting of attention in the Posner task. Cerebral cortex (New York, N.Y. : 1991) 20, 1574-1585.

Eriksen, C.W., St. James, J.D., 1986. Visual attention within and around the field of focal attention: A zoom lens model. Perception \& Psychophysics 40, 225-240.

Eriksen, C.W., Yeh, Y., 1985. Allocation of attention in the visual field. Journal of Experimental Psychology: Human Perception and Performance 11, 582-597.

Feldman, H., Friston, K.J., 2010. Attention, uncertainty, and free-energy. Front Hum Neurosci 4, 215.

Fuller, S., Park, Y., Carrasco, M., 2009. Cue contrast modulates the effects of exogenous attention on appearance. Vision Res 49, 1825-1837.

Giordano, A.M., McElree, B., Carrasco, M., 2009. On the automaticity and flexibility of covert attention: a speed-accuracy trade-off analysis. J Vis 9, 30.31-10. 
Gould, I.C., Rushworth, M.F., Nobre, A.C., 2011. Indexing the graded allocation of visuospatial attention using anticipatory alpha oscillations. J Neurophysiol 105, 13181326.

Gould, I.C., Wolfgang, B.J., Smith, P.L., 2007. Spatial uncertainty explains exogenous and endogenous attentional cuing effects in visual signal detection. J Vis 7, 4.1-17.

Gray, R., 2000. Attentional modulation of motion-in-depth processing. Vision Research 40, 1041-1050.

Graziano, M.S.A., Andersen, R.A., Snowden, R.J., 1994. Tuning of MST neurons to spiral motions. Journal of Neuroscience 14, 54-67.

Jans, B., Peters, J.C., De Weerd, P., 2010. Visual spatial attention to multiple locations at once: the jury is still out. Psychological Review 117, 637-684.

Karni, A., Sagi, D., 1991. Where practice makes perfect in texture discrimination: Evidence for primary visual cortex plasticity. Proceedings of the National Academy of Sciences 88, 4966-4970.

Kingdom, F.A.A., Prins, N., 2010. Psychophysics - A Practical Introduction. Academic Press.

Liu, T., Fuller, S., Carrasco, M., 2006. Attention alters the appearance of motion coherence. Psychonomic Bulletin \& Review 13, 1091-1096.

McMains, S.A., Somers, D.C., 2004. Multiple spotlights of attentional selection in human visual cortex. Neuron 42, 677-686.

McMains, S.A., Somers, D.C., 2005. Processing efficiency of divided spatial attention mechanisms in human visual cortex. Journal of Neuroscience 25, 9444-9448.

Montagna, B., Pestilli, F., Carrasco, M., 2009. Attention trades off spatial acuity. Vision Research 49, 735-745.

Morawetz, C., Holz, P., Baudewig, J., Treue, S., Dechent, P., 2007. Split of attentional resources in human visual cortex. Visual Neuroscience 24, 817-826.

Pashler, H., 1993. Doing two things at the same time. American Scientist 81, 48-55.

Pashler, H., 1994. Dual-task interference in simple tasks: data and theory. Psychological Bulletin 116, 220-244.

Pastukhov, A., Fischer, L., Braun, J., 2009. Visual attention is a single, integrated resource. Vision Research 49, 1166-1173.

Pestilli, F., Carrasco, M., 2005. Attention enhances contrast sensitivity at cued and impairs it at uncued locations. Vision Research 45, 1867-1875.

Posner, M.I., 1980. Orienting of attention. Quarterly Journal of Experimental Psychology 32, 3-25.

Posner, M.I., Snyder, C.R.R., Davidson, B.J., 1980. Attention and the detection of signals. JEP-General 109, 160-174.

Richard D. Wright, L.M.W., 2008. Orienting of Attention. Oxford University Press. 
Sperling, G., Melchner, M.J., 1978. The attention operating characteristic: Examples from visual search. Science 202, 315-318.

Spitzer, H., Desimone, R., Moran, J., 1988. Increased attention enhances both behavioral and neuronal performance. Science 240, 338-340.

Treue, S., Martinez-Trujillo, J.C., 2012. The spotlight of attention: shifting, resizing and splitting receptive fields when processing visual motion. e-Neuroforum 3, 74-79.

Treue, S., Maunsell, J.H.R., 1999. Effects of attention on the processing of motion in macaque middle temporal and medial superior temporal visual cortical areas. Journal of Neuroscience 19, 7591-7602.

Verghese, A., Anderson, A.J., Vidyasagar, T.R., 2013. Space, color, and direction of movement: how do they affect attention? J Vis 13.

Yeshurun, Y., Carrasco, M., 1998. Attention improves or impairs visual performance by enhancing spatial resolution. Nature 396, 72-75.

Yeshurun, Y., Carrasco, M., 1999. Spatial attention improves performance in spatial resolution tasks. Vision Research 39, 293-306.

Zizlsperger, L., Sauvigny, T., Haarmeier, T., 2012. Selective attention increases choice certainty in human decision making. PLoS One 7, e41136. 


\title{
Supplementary Material for
}

\section{Graded visual attention in discriminating human spiral motion patterns}

\author{
Sepideh Fazeli ${ }^{1}$, Stefan Treue ${ }^{1,2}$ \\ ${ }^{1}$ Cognitive Neuroscience Laboratory, German Primate Center, Goettingen, Germany \\ ${ }^{2}$ Bernstein Center for Computational Neuroscience, Goettingen, Germany
}

\section{SUPPLEMENTARY FIGURES}

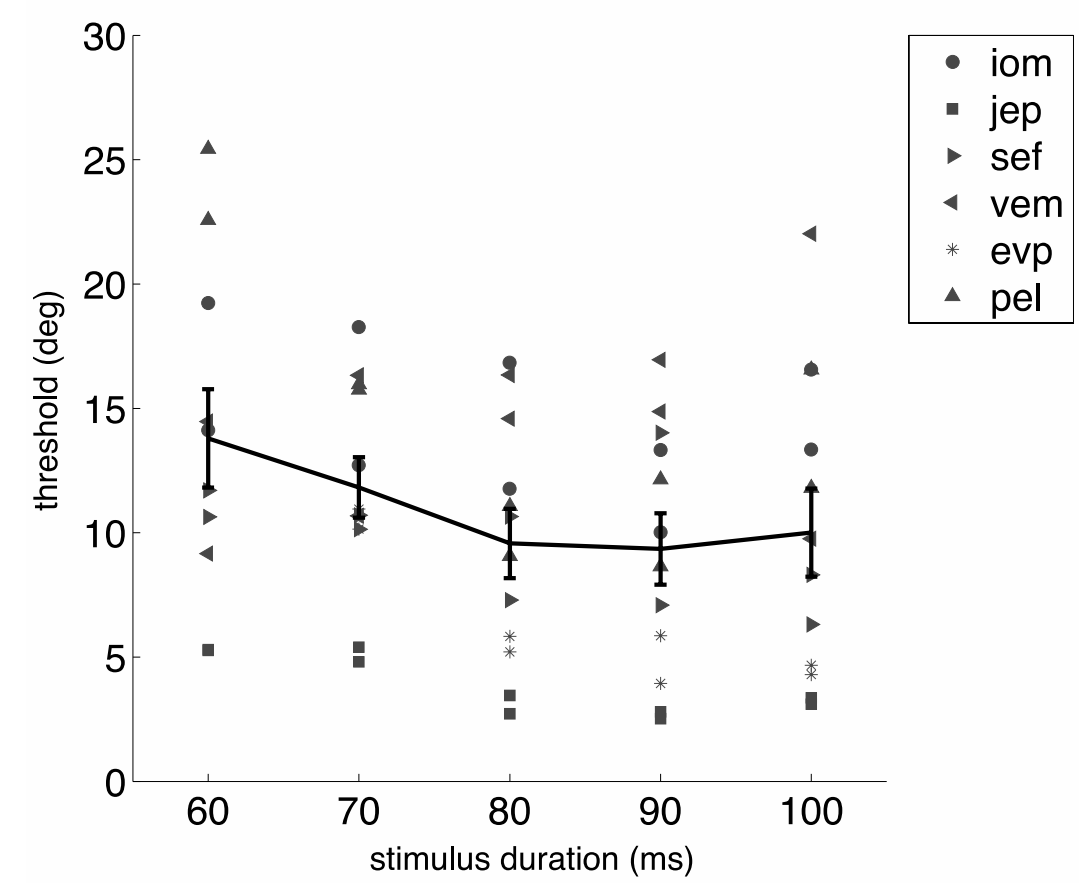

Sup.Fig.1: Direction discrimination thresholds as a function of stimulus duration across six subjects

The averaged thresholds across left and right targets are plotted in five stimulus durations of $60 \mathrm{~ms}$, $70 \mathrm{~ms}, 80 \mathrm{~ms}, 90 \mathrm{~ms} \& 100 \mathrm{~ms}$ for $100 \%$ valid cue in experiment 1 (pre-cued). The black solid line represents the averaged threshold across six subjects. Error bars represent the standard error. The averaged threshold curve increased about $80 \mathrm{~ms}$. 


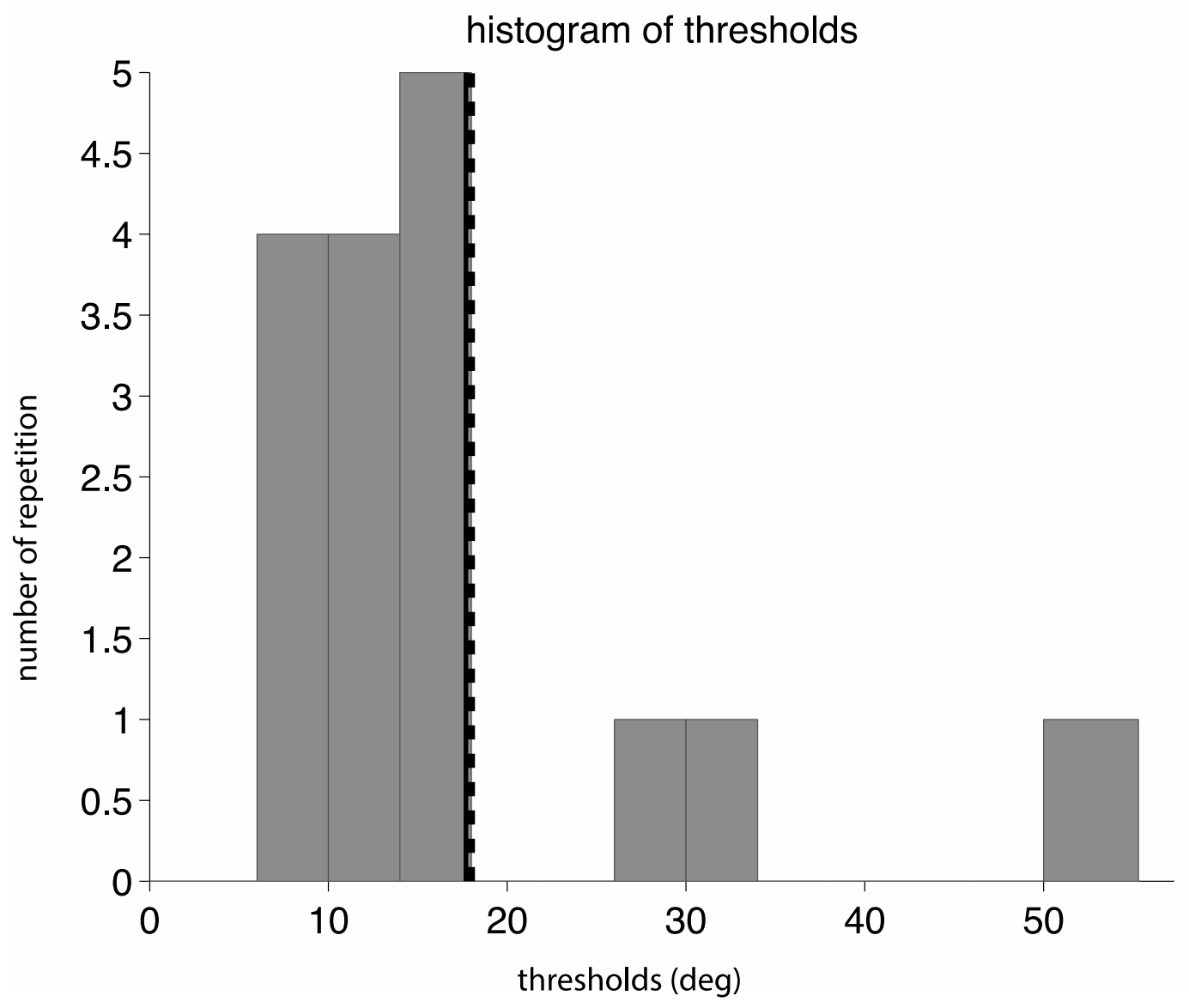

Sup.Fig.2: Threshold distribution across six subjects for $100 \%$ attention condition in the experiment one

Histogram of the determined thresholds of both left and right targets for $100 \%$ valid cue condition is plotted. The histogram has the mean of $17.3^{\circ}$ with the standard deviation of $12.14^{\circ}$. The cut off threshold is set to $18^{\circ}$ and marked with the dashed line. 84th percentile is $17.7^{\circ}$ and marked with solid line. 


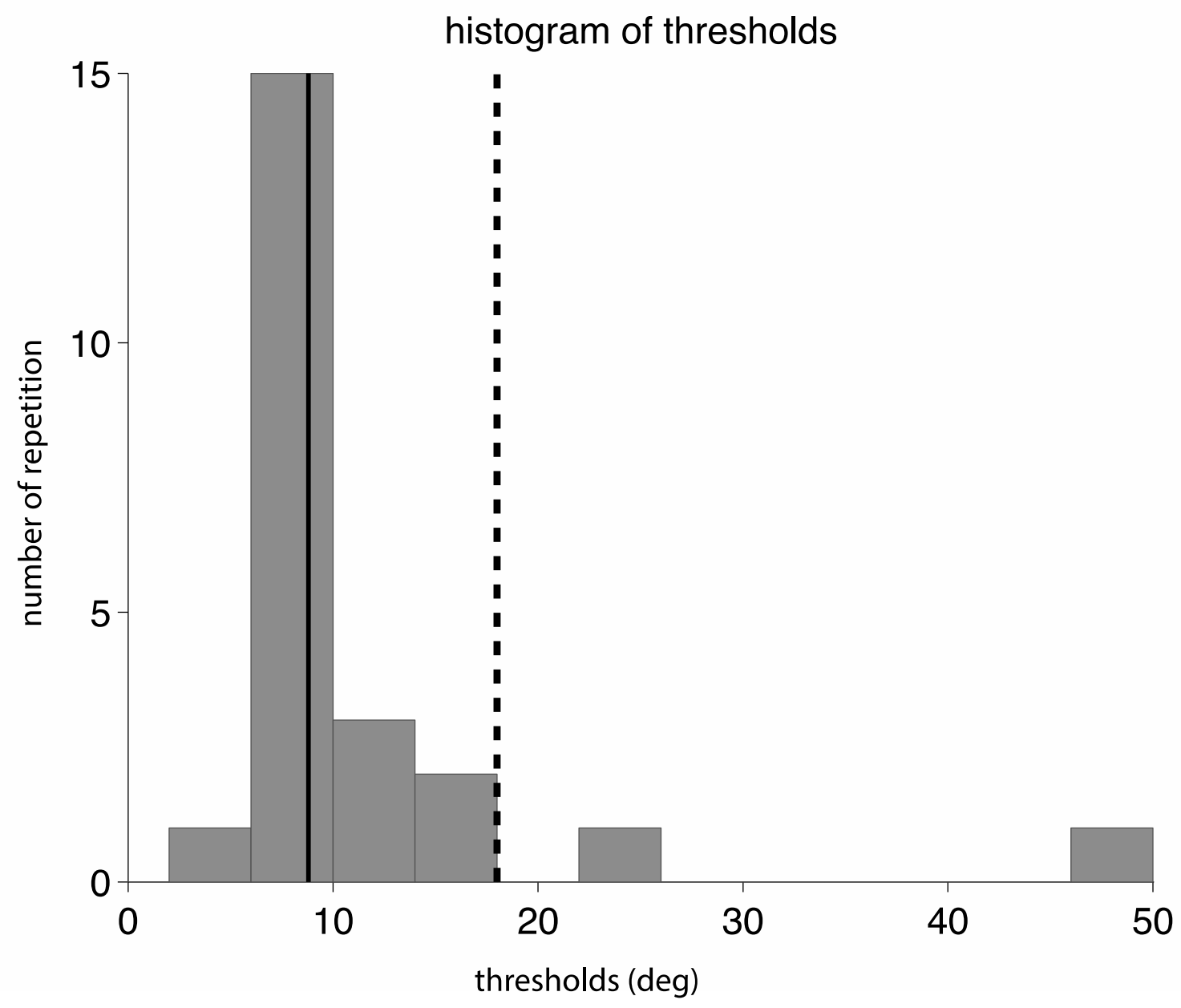

Sup.Fig.3: Threshold distribution across six subjects for $100 \%$ attention condition in the experiment two

Histogram of the determined thresholds is plotted in same way as Sup-Fig 2. The histogram has the mean of $11.5^{\circ}$ with the standard deviation of $8.8^{\circ}$. The cut off threshold is set to $18^{\circ}$ and marked with the dashed line. 84th percentile is $8.8^{\circ}$ and marked with the solid line. 
A

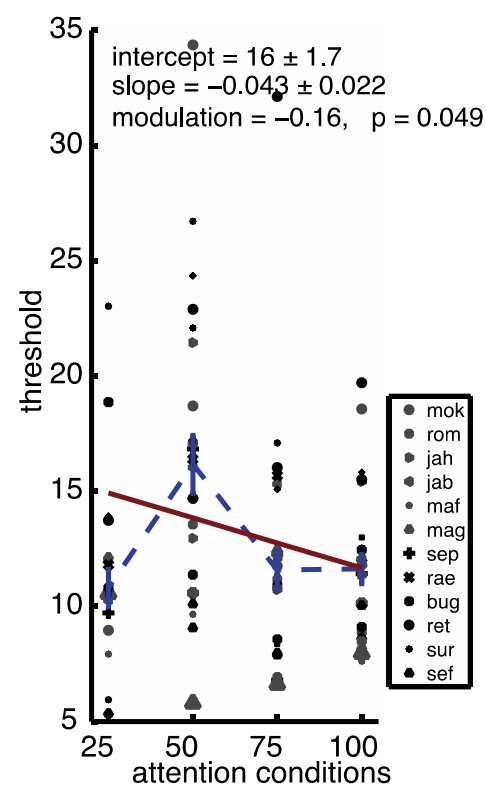

B

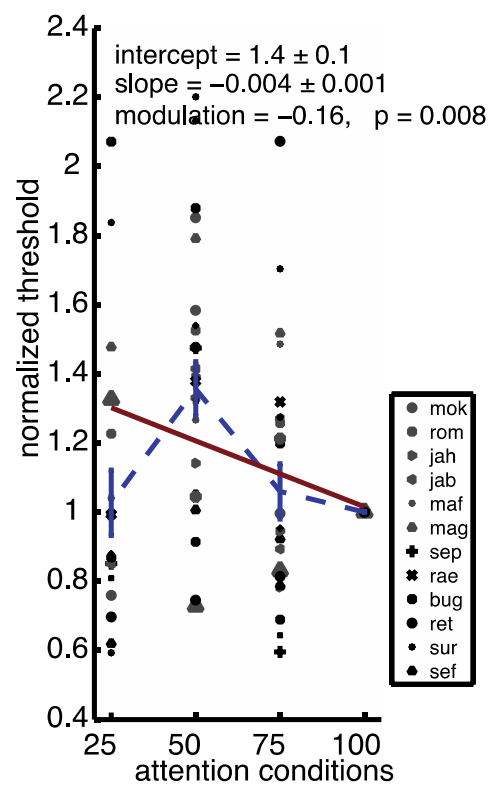

C

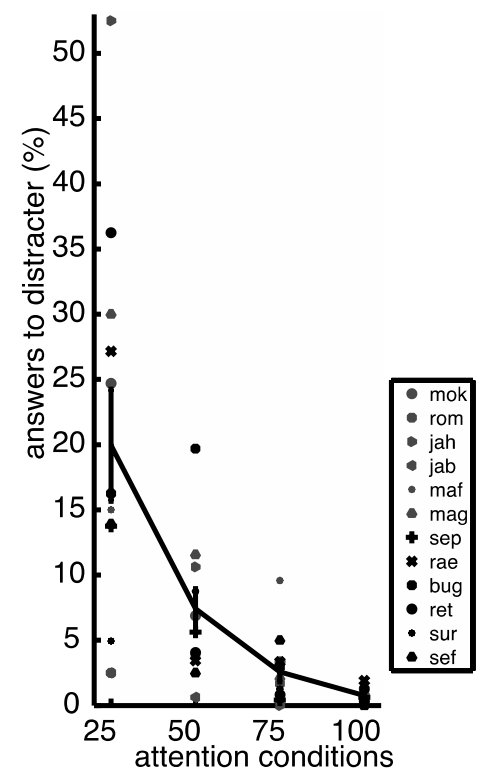

\section{Sup.Fig.4: Graded attention in discriminating spiral patterns in the no mask pilot experiment}

(A) The extracted thresholds from psychometric functions in each attention condition are plotted in a same way as Fig. 4A. There was a significant difference between the threshold in the four attention conditions ( $F=8.06, p=0.0008$ rm-ANOVA). The weighted fitted line to the thresholds had the intercept $=16 \pm 1.7$ and the slope $=-0.04 \pm 0.02$. The general linear model showed $-9 \%$ threshold modulation by halving the amount of allocated attention $(p=0.04)$. (B) The extracted thresholds from psychometric functions in each attention condition are plotted with normalization in a same way as Fig. 4B. Repeated measured ANOVA revealed no significant difference between the threshold in the four attention conditions $(F=8.48, p=0.0003$ rm-ANOVA). The weighted fitted line to the thresholds had the intercept $=1.4 \pm 0.1$ and the slope $=-0.004 \pm 0.001$. The general linear model showed - $9 \%$ threshold modulation by halving the amount of allocated attention $(p=0.008)$. (C) The percentage of the answers to the distorter are plotted in each attentional conditions. The black solid line represents the average of them across twelve subjects. Error bars represent the standard error of the mean. 
$\mathrm{X}-\mathrm{Y}$ plot of thresholds for left and right stimuli

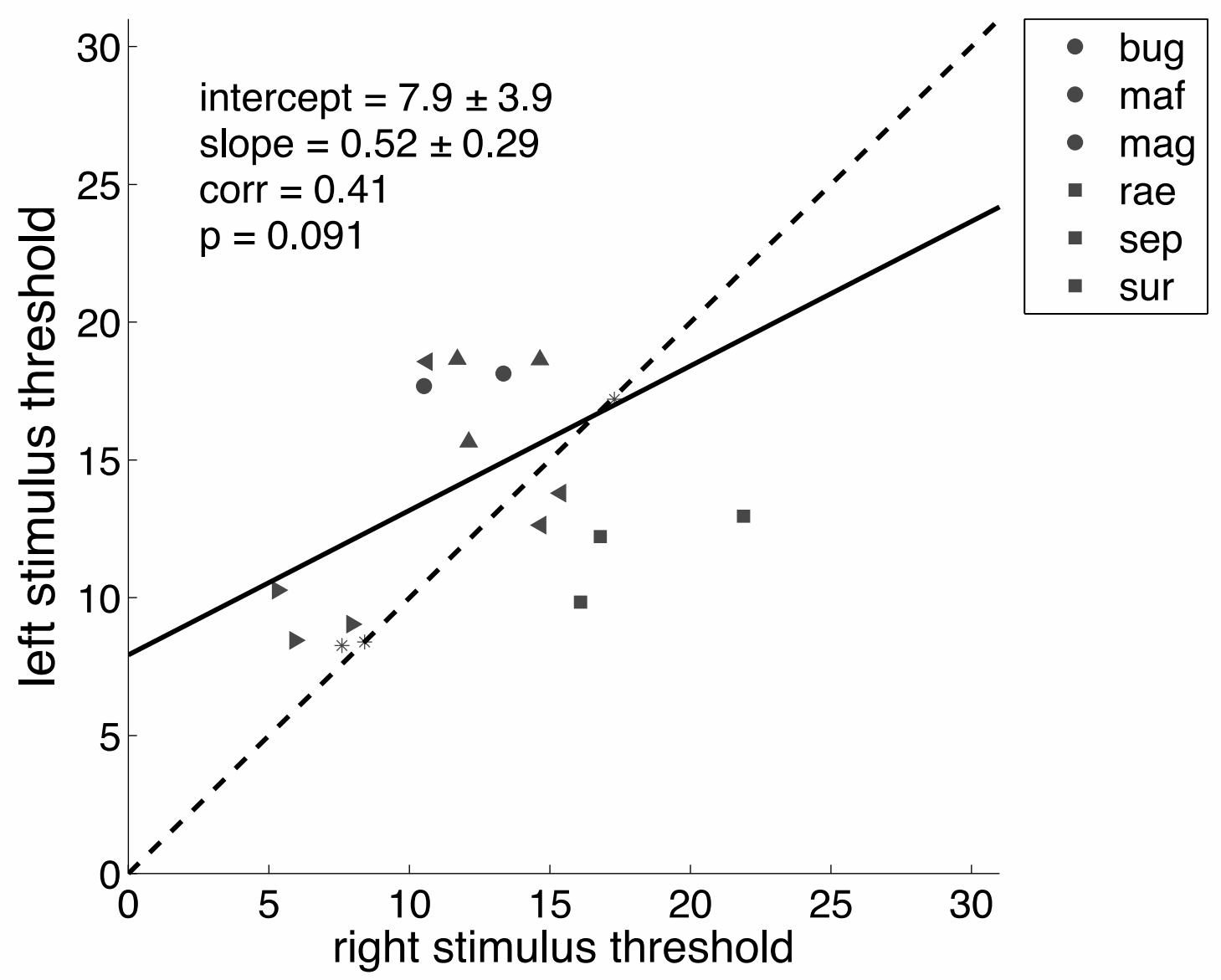

Sup.Fig.5: scatter plot of the determined thresholds for left and right stimuli in the across all attention conditions in experiment one

For each subject for each attention condition, his/her threshold for the left target is plotted on the $y$ axis whereas the threshold for right-sided target is plotted on the $x$-axis. The fitted line to the all data point has the intercept of $7.9 \pm 3.9$ (mean $\pm S E$ ) with the $p=0.06$ and its slope is $0.52 \pm 0.29$ $(p=0.09)$. 


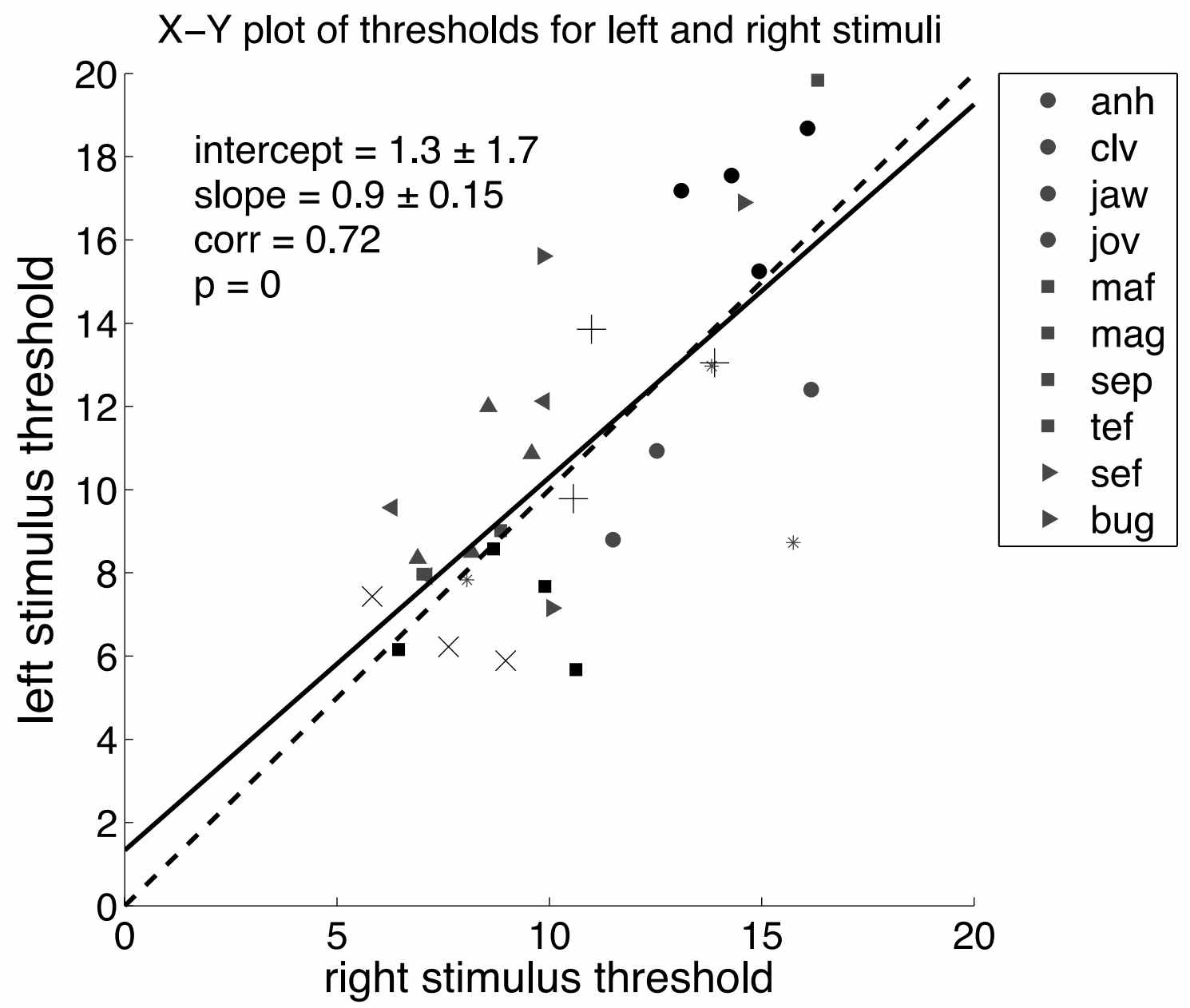

Sup.Fig.6: scatter plot of the determined thresholds for left and right stimuli across all attention conditions in experiment two

$\mathrm{X}-\mathrm{Y}$ plot of the left-stimulus and right-stimulus thresholds is plotted in same way as sup-Fig. 5. The fitted line to the all data point has the intercept of $1.3 \pm 1.7(p=0.44)$ and its slope is $0.9 \pm 0.15$ (mean $\pm S E, p<0.01)$. Moreover, the left- stimulus and right- stimulus thresholds show a significant linear correlation $(p<0.01)$. 


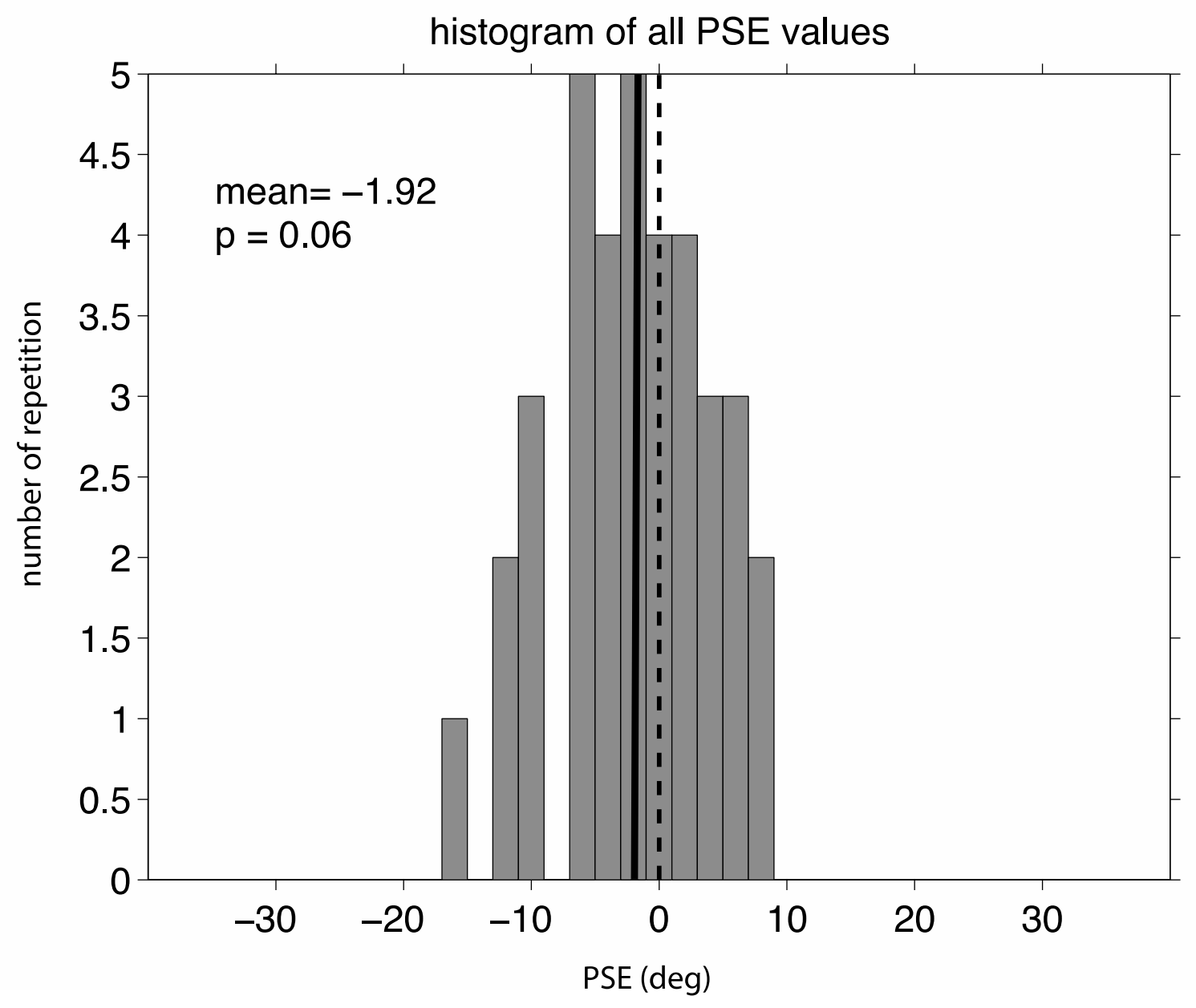

Sup.Fig.7: PSEs distribution across all attention conditions in experiment one

Histogram of the determined PSEs for all attention conditions in a population of six subjects is plotted. The histogram has the mean of $-1.9^{\circ}$, indicated by solid line. The histogram has no significant bias for any spiral direction (Wilcoxon signed rank test, $p=0.09$ ) 


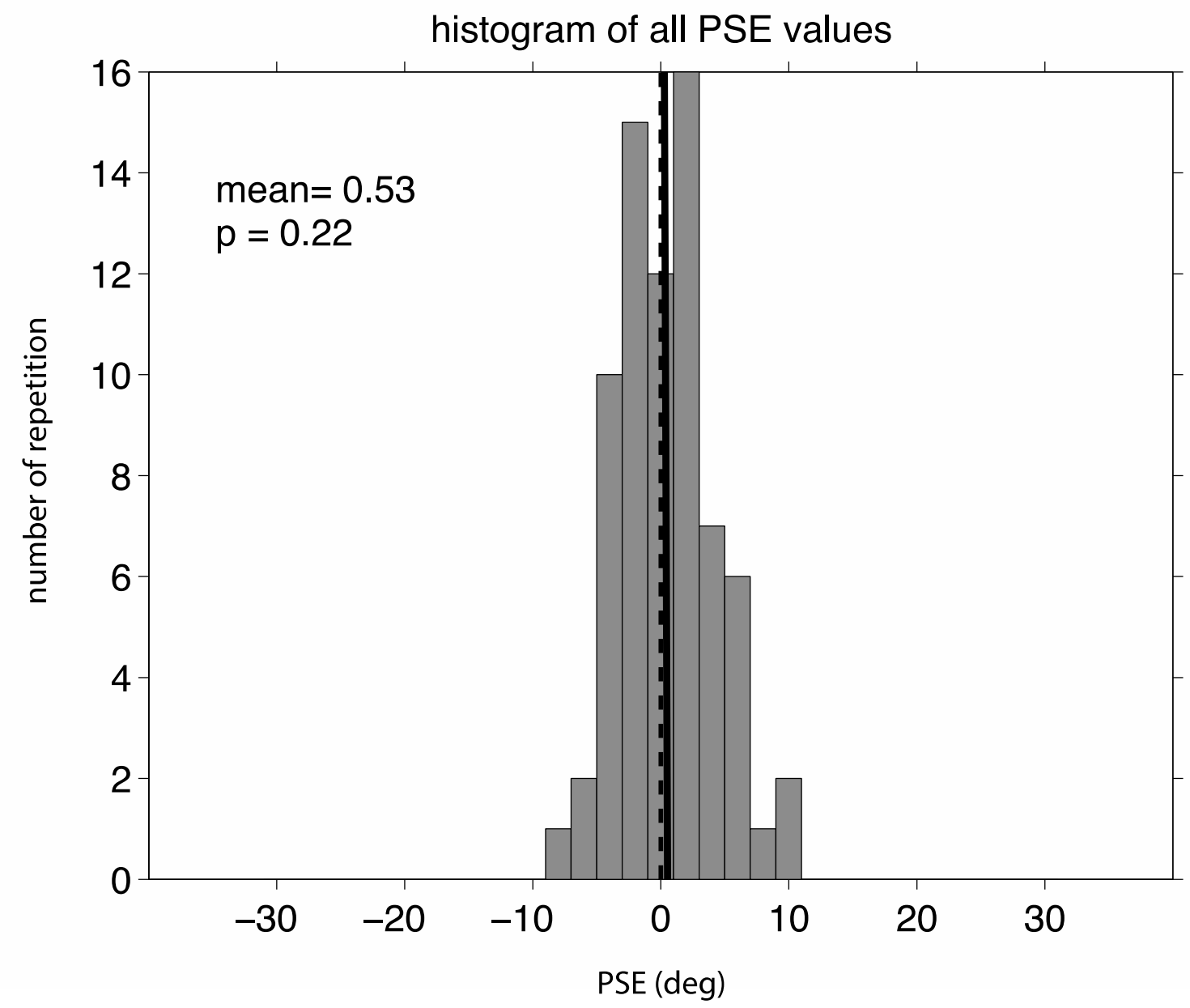

Sup.Fig.8: PSEs distribution across all attention conditions in experiment two

Histogram of the determined PSEs for all attention conditions in a population of ten subjects is plotted. The histogram has the mean of $0.53^{\circ}$, indicated by solid line. The histogram has no significant bias for any spiral direction (Wilcoxon signed rank test, $p=0.3$ ) 


\section{SUPPLEMENTARY TABLES}

The following tables summarized the extracted parameters from the fits to the psychometric functions in all conditions for each individual subject in both experiments. The fitting routine had a grid of seed values to achive the best fit and avoid stucking in local minima. The search grids for alpha and beta were -3:0.1:3 and 0.1:0.5:5 respectively for all fits. The bootstrap had 400 iterations to estimate the confidence intervals and maximum-likelihood values.

\section{List of abbreviation}

thr: threshold, SE: standard error, PSE: point of subjective equality, IT:included trials, AT: all trials, MLL: maximum-likelihooh, SSE: sum of squared error, R2: R-squared, M-Corr: correlation between the mean of the data points and the fitted values to them.

\section{Experiment 1}

bug_1_9-RSM-table.txt

$\begin{array}{lcccccccccc} & \text { thr } & \pm S E & \text { PSE } & \pm S E & \text { IT } & \text { AT } & \text { MLL } & \text { SSE } & \text { R2 } & \text { M-Corr } \\ \text { 100\%-L } & 17.69 & 3.56 & 4.64 & 1.91 & 158.00 & 158.00 & 0.76 & 0.20 & 0.44 & 0.94 \\ 100 \%-\mathrm{R} & 10.51 & 1.75 & -11.30 & 1.18 & 159.00 & 160.00 & 0.46 & 0.19 & 0.52 & 0.92 \\ 75 \%-\mathrm{L} & 18.13 & 4.31 & 1.22 & 2.32 & 118.00 & 120.00 & 0.78 & 0.20 & 0.46 & 0.90 \\ 75 \%-\mathrm{R} & 13.36 & 3.30 & -9.88 & 1.77 & 113.00 & 120.00 & 0.51 & 0.20 & 0.48 & 0.89 \\ 50 \%-\mathrm{L} & 31.29 & 16.01 & 6.80 & 5.89 & 84.00 & 158.00 & 0.14 & 0.23 & 0.32 & 0.70 \\ 50 \%-\mathrm{R} & 16.60 & 4.63 & -12.28 & 2.87 & 92.00 & 162.00 & 0.47 & 0.19 & 0.48 & 0.77 \\ 25 \%-\mathrm{L} & \mathrm{NaN} & \mathrm{NaN} & \mathrm{NaN} & \mathrm{NaN} & 5.00 & 40.00 & \mathrm{NaN} & \mathrm{NaN} & \mathrm{NaN} & \mathrm{NaN} \\ 25 \%-\mathrm{R} & 1.00 & 0.00 & -0.57 & 0.00 & 5.00 & 81.00 & 0.00 & 0.00 & 1.00 & 1.00 \\ 50 \%-\mathrm{L}-\mathrm{V} & 43.46 & 163.87 & 3.58 & 37.28 & 42.00 & 79.00 & 0.21 & 0.25 & 0.25 & 0.57 \\ 50 \%-\mathrm{R}-\mathrm{V} & 15.96 & 6.48 & -12.07 & 4.43 & 54.00 & 81.00 & 0.33 & 0.20 & 0.48 & 0.71 \\ 50 \%-\mathrm{L}-\mathrm{IV} & \mathrm{NaN} & \mathrm{NaN} & \mathrm{NaN} & \mathrm{NaN} & 5.00 & 79.00 & \mathrm{NaN} & \mathrm{NaN} & \mathrm{NaN} & \mathrm{NaN} \\ 50 \%-\mathrm{R}-\mathrm{IV} & 1.00 & 0.00 & -0.57 & 0.00 & 0.00 & 0.00 & 0.00 & 0.00 & 1.00 & 1.00\end{array}$


mag_1_4-RSM-table.txt

$\begin{array}{lcccccccccc} & \text { thr } & \pm S E & \text { PSE } & \pm S E & \text { IT } & \text { AT } & \text { MLL } & \text { SSE } & \text { R2 } & \text { M-Corr } \\ 100 \%-L & 9.04 & 1.55 & -2.17 & 1.03 & 157.00 & 160.00 & 0.22 & 0.19 & 0.52 & 0.96 \\ 100 \%-R & 7.95 & 1.45 & 5.25 & 0.84 & 159.00 & 160.00 & 0.78 & 0.20 & 0.47 & 0.98 \\ 75 \%-L & 10.28 & 2.63 & -1.64 & 1.58 & 90.00 & 120.00 & 0.89 & 0.20 & 0.49 & 0.95 \\ 75 \%-R & 5.31 & 1.33 & 0.15 & 0.88 & 74.00 & 120.00 & 0.45 & 0.18 & 0.56 & 0.97 \\ 50 \%-L & 8.45 & 2.53 & 0.16 & 1.70 & 62.00 & 160.00 & 0.69 & 0.19 & 0.52 & 0.78 \\ 50 \%-R & 5.93 & 1.31 & 1.41 & 1.04 & 72.00 & 160.00 & 0.54 & 0.16 & 0.62 & 0.96 \\ 25 \%-L & 55.00 & 77.00 & -30.35 & 61.79 & 7.00 & 40.00 & 0.00 & 0.35 & 0.10 & 0.59 \\ 25 \%-R & 0.00 & 0.00 & -9.00 & 0.94 & 10.00 & 80.00 & 0.50 & 0.07 & 0.83 & 1.00 \\ 50 \%-L-V & 9.86 & 5.62 & -0.06 & 2.76 & 44.00 & 80.00 & 0.70 & 0.21 & 0.46 & 0.77 \\ 50 \%-R-V & 5.88 & 1.75 & 2.27 & 1.35 & 40.00 & 80.00 & 0.53 & 0.17 & 0.61 & 0.93 \\ 50 \%-L-I V & 55.00 & 77.00 & -30.35 & 61.79 & 7.00 & 80.00 & 0.00 & 0.35 & 0.10 & 0.59 \\ 50 \%-R-I V & 0.00 & 0.00 & -9.00 & 0.94 & 0.00 & 0.00 & 0.50 & 0.07 & 0.83 & 1.00\end{array}$

maf_1_6-RSM-table.txt

thr \pm SE PSE \pm SE IT AT MLL SSE R2 M-Corr

$\begin{array}{lllllllllll}100 \%-\mathrm{L} & 12.22 & 2.63 & -2.34 & 1.41 & 153.00 & 154.00 & 0.63 & 0.21 & 0.41 & 0.92\end{array}$

$\begin{array}{lllllllllll}100 \%-R & 16.80 & 2.97 & 5.07 & 1.83 & 156.00 & 160.00 & 0.14 & 0.19 & 0.49 & 0.78\end{array}$

$\begin{array}{lllllllllll}75 \%-\mathrm{L} & 9.84 & 2.33 & -1.38 & 1.37 & 106.00 & 117.00 & 0.29 & 0.20 & 0.47 & 0.88\end{array}$

$\begin{array}{lllllllllll}75 \%-R & 16.09 & 3.38 & 8.64 & 2.23 & 118.00 & 120.00 & 0.97 & 0.20 & 0.48 & 0.94\end{array}$

$\begin{array}{lllllllllll}50 \%-\mathrm{L} & 12.96 & 3.01 & 2.88 & 1.73 & 107.00 & 156.00 & 0.91 & 0.20 & 0.46 & 0.95\end{array}$

$\begin{array}{lllllllllll}50 \%-\mathrm{R} & 21.89 & 7.47 & 8.31 & 3.56 & 105.00 & 164.00 & 0.94 & 0.21 & 0.42 & 0.81\end{array}$

25\%-L NaN NaN NaN NaN $1.00 \quad 40.00 \quad \mathrm{NaN} N a N \quad \mathrm{NaN} N$

$\begin{array}{lllllllllll}25 \%-R & 1.00 & 0.00 & -0.57 & 0.00 & 1.00 & 83.00 & 0.00 & 0.00 & 1.00 & 1.00\end{array}$

$\begin{array}{lllllllllll}50 \%-L-V & 14.00 & 7.47 & 2.87 & 2.56 & 59.00 & 77.00 & 0.72 & 0.22 & 0.42 & 0.82\end{array}$

$\begin{array}{lllllllllll}50 \%-R-V & 28.60 & 28.57 & 8.72 & 7.86 & 56.00 & 81.00 & 1.00 & 0.22 & 0.38 & 0.79\end{array}$

50\%-L-IV NaN NaN NaN NaN $1.00 \quad 79.00 \quad \mathrm{NaN} \quad \mathrm{NaN} \quad \mathrm{NaN} \quad \mathrm{NaN}$

$\begin{array}{lllllllllll}50 \% \text {-R-IV } & 1.00 & 0.00 & -0.57 & 0.00 & 0.00 & 0.00 & 0.00 & 0.00 & 1.00 & 1.00\end{array}$ 
rae_1_8-RSM-table.txt

$\begin{array}{lcccccccccc} & \text { thr } & \pm S E & \text { PSE } & \pm S E & \text { IT } & \text { AT } & \text { MLL } & \text { SSE } & \text { R2 } & \text { M-Corr } \\ 100 \%-L & 13.80 & 2.44 & -3.86 & 1.65 & 145.00 & 148.00 & 0.66 & 0.19 & 0.49 & 0.91 \\ 100 \%-R & 15.39 & 3.43 & -3.63 & 1.80 & 141.00 & 160.00 & 0.32 & 0.21 & 0.44 & 0.88 \\ 75 \%-L & 12.64 & 3.79 & -3.73 & 1.85 & 108.00 & 118.00 & 0.36 & 0.21 & 0.44 & 0.86 \\ 75 \%-R & 14.67 & 4.11 & -3.99 & 2.04 & 108.00 & 121.00 & 0.89 & 0.21 & 0.41 & 0.90 \\ 50 \%-L & 18.57 & 4.99 & -5.34 & 2.90 & 93.00 & 158.00 & 0.56 & 0.20 & 0.46 & 0.84 \\ 50 \%-R & 10.63 & 2.47 & -5.92 & 1.40 & 118.00 & 162.00 & 0.30 & 0.19 & 0.51 & 0.91 \\ 25 \%-L & 184.00 & 61.00 & -20.63 & 30.83 & 9.00 & 39.00 & 0.00 & 0.37 & 0.04 & 0.62 \\ 25 \%-R & 11.00 & 16.00 & 2.06 & 10.34 & 8.00 & 81.00 & 0.00 & 0.28 & 0.51 & 0.81 \\ 50 \%-L-V & 14.32 & 4.23 & -8.52 & 3.07 & 52.00 & 79.00 & 0.64 & 0.19 & 0.54 & 0.89 \\ 50 \%-R-V & 6.07 & 1.78 & -7.12 & 1.15 & 59.00 & 81.00 & 0.53 & 0.18 & 0.55 & 0.95 \\ 50 \%-L-I V & 184.00 & 61.00 & -20.63 & 30.83 & 9.00 & 79.00 & 0.00 & 0.37 & 0.04 & 0.62 \\ 50 \%-R-I V & 11.00 & 16.00 & 2.06 & 10.34 & 0.00 & 0.00 & 0.00 & 0.28 & 0.51 & 0.81\end{array}$

sep_1_6-RSM-table.txt

$\begin{array}{lcccccccccc} & \text { thr } & \pm S E & \text { PSE } & \pm S E & \text { IT } & \text { AT } & \text { MLL } & \text { SSE } & \text { R2 } & \text { M-Corr } \\ 100 \%-L & 8.27 & 1.31 & 0.16 & 0.89 & 159.00 & 159.00 & 0.80 & 0.19 & 0.51 & 0.98 \\ 100 \%-R & 7.60 & 1.25 & 1.77 & 0.91 & 157.00 & 160.00 & 0.27 & 0.19 & 0.52 & 0.97 \\ 75 \%-L & 8.40 & 1.66 & -1.68 & 1.10 & 110.00 & 117.00 & 0.82 & 0.18 & 0.53 & 0.95 \\ 75 \%-R & 8.41 & 1.93 & 3.25 & 1.25 & 109.00 & 120.00 & 1.00 & 0.19 & 0.50 & 0.99 \\ 50 \%-L & 17.20 & 3.82 & -0.15 & 2.17 & 127.00 & 159.00 & 0.84 & 0.20 & 0.45 & 0.92 \\ 50 \%-R & 17.30 & 5.92 & 3.06 & 2.84 & 85.00 & 161.00 & 0.44 & 0.22 & 0.41 & 0.77 \\ 25 \%-L & \text { NaN } & \text { NaN } & \text { NaN } & \text { NaN } & 3.00 & 40.00 & \text { NaN } & \text { NaN } & \text { NaN } & \text { NaN } \\ 25 \%-R & 2.00 & 2.00 & 7.24 & 2.10 & 11.00 & 80.00 & 0.27 & 0.16 & 0.73 & 1.00 \\ 50 \%-L-V & 9.51 & 2.64 & -0.92 & 1.45 & 71.00 & 80.00 & 0.93 & 0.20 & 0.49 & 0.95 \\ 50 \%-R-V & 17.72 & 12.85 & 2.08 & 3.69 & 60.00 & 81.00 & 0.44 & 0.22 & 0.38 & 0.83 \\ 50 \%-L-I V & N a N & N a N & N a N & N a N & 3.00 & 79.00 & \text { NaN } & \text { NaN } & \text { NaN } & \text { NaN } \\ 50 \%-R-I V & 2.00 & 2.00 & 7.24 & 2.10 & 0.00 & 0.00 & 0.27 & 0.16 & 0.73 & 1.00\end{array}$


sur_1_6-RSM-table.txt

$\begin{array}{lcccccccccc} & \text { thr } & \pm S E & \text { PSE } & \pm S E & \text { IT } & \text { AT } & \text { MLL } & \text { SSE } & \text { R2 } & \text { M-Corr } \\ 100 \%-L & 15.65 & 3.26 & -5.27 & 1.74 & 159.00 & 159.00 & 0.98 & 0.20 & 0.46 & 0.96 \\ 100 \%-R & 12.11 & 2.01 & -9.07 & 1.36 & 158.00 & 160.00 & 0.73 & 0.20 & 0.47 & 0.96 \\ 75 \%-L & 18.63 & 5.57 & -6.77 & 2.66 & 101.00 & 118.00 & 0.91 & 0.21 & 0.42 & 0.91 \\ 75 \%-R & 14.65 & 3.43 & -5.04 & 1.94 & 117.00 & 120.00 & 0.61 & 0.20 & 0.47 & 0.87 \\ 50 \%-L & 18.66 & 4.10 & -10.92 & 2.46 & 99.00 & 158.00 & 0.83 & 0.19 & 0.50 & 0.92 \\ 50 \%-R & 11.71 & 2.08 & -15.65 & 1.59 & 122.00 & 162.00 & 0.72 & 0.16 & 0.60 & 0.97 \\ 25 \%-L & \text { NaN } & \text { NaN } & \text { NaN } & \text { NaN } & 3.00 & 40.00 & \text { NaN } & \text { NaN } & \text { NaN } & \text { NaN } \\ 25 \%-R & 35.00 & 261.00 & -17.49 & 28.78 & 25.00 & 80.00 & 0.05 & 0.24 & 0.37 & 0.60 \\ 50 \%-L-V & 21.53 & 7.49 & -9.56 & 3.97 & 64.00 & 80.00 & 0.83 & 0.20 & 0.47 & 0.95 \\ 50 \%-R-V & 9.29 & 2.23 & -15.44 & 1.66 & 65.00 & 82.00 & 0.32 & 0.16 & 0.61 & 0.94 \\ 50 \%-L-I V & N a N & N a N & N a N & N a N & 3.00 & 78.00 & \text { NaN } & \text { NaN } & \text { NaN } & \text { NaN } \\ 50 \%-R-I V & 35.00 & 261.00 & -17.49 & 28.78 & 0.00 & 0.00 & 0.05 & 0.24 & 0.37 & 0.60\end{array}$




\section{Experiment 2}

anh_1_2-@SM-table.txt

$\begin{array}{lcccccccccc} & \text { thr } & \pm S E & \text { PSE } & \pm S E & \text { IT } & \text { AT } & \text { MLL } & \text { SSE } & \text { R2 } & \text { M-Corr } \\ 100 \%-L & 8.79 & 1.61 & 0.33 & 1.00 & 160.00 & 160.00 & 0.65 & 0.19 & 0.50 & 0.96 \\ 100 \%-R & 11.50 & 2.13 & -3.68 & 1.26 & 159.00 & 160.00 & 0.48 & 0.20 & 0.46 & 0.94 \\ 75 \%-L & 10.92 & 2.69 & 1.63 & 1.46 & 120.00 & 120.00 & 0.50 & 0.20 & 0.47 & 0.89 \\ 75 \%-R & 12.53 & 2.62 & -8.43 & 1.69 & 119.00 & 121.00 & 0.69 & 0.19 & 0.52 & 0.92 \\ 50 \%-L & 12.40 & 2.41 & -0.85 & 1.31 & 160.00 & 160.00 & 0.40 & 0.21 & 0.44 & 0.86 \\ 50 \%-R & 16.17 & 3.08 & 0.81 & 1.69 & 159.00 & 160.00 & 0.81 & 0.21 & 0.42 & 0.93 \\ 25 \%-L & 12.00 & 127.00 & -2.32 & 46.26 & 39.00 & 39.00 & 0.17 & 0.22 & 0.43 & 0.58 \\ 25 \%-R & 9.00 & 7.00 & 0.26 & 2.62 & 39.00 & 40.00 & 0.35 & 0.21 & 0.47 & 0.87 \\ 50 \%-L-V & 11.30 & 3.41 & -1.15 & 1.93 & 80.00 & 80.00 & 0.29 & 0.21 & 0.46 & 0.88 \\ 50 \%-R-V & 12.64 & 5.64 & -1.29 & 2.18 & 79.00 & 80.00 & 1.00 & 0.22 & 0.41 & 0.95 \\ 50 \%-L-I V & 11.62 & 127.12 & -2.32 & 46.26 & 39.00 & 80.00 & 0.17 & 0.22 & 0.43 & 0.58 \\ 50 \%-R-I V & 8.75 & 7.41 & 0.26 & 2.62 & 39.00 & 80.00 & 0.35 & 0.21 & 0.47 & 0.87\end{array}$

\section{bug_1_11-@SM-table.txt}

$\begin{array}{lcccccccccc} & \text { thr } & \pm S E & \text { PSE } & \pm S E & \text { IT } & \text { AT } & \text { MLL } & \text { SSE } & \text { R2 } & \text { M-Corr } \\ 100 \%-L & 17.55 & 3.24 & 3.63 & 2.04 & 160.00 & 160.00 & 0.88 & 0.20 & 0.46 & 0.95 \\ 100 \%-R & 14.29 & 2.70 & -4.65 & 1.59 & 159.00 & 160.00 & 0.93 & 0.20 & 0.44 & 0.96 \\ 75 \%-L & 18.69 & 4.80 & 2.90 & 2.33 & 120.00 & 120.00 & 0.94 & 0.21 & 0.42 & 0.93 \\ 75 \%-R & 16.09 & 3.55 & -2.30 & 2.03 & 120.00 & 120.00 & 0.84 & 0.20 & 0.45 & 0.96 \\ 50 \%-L & 17.19 & 3.24 & 2.32 & 2.00 & 158.00 & 159.00 & 0.97 & 0.20 & 0.45 & 0.96 \\ 50 \%-R & 13.11 & 2.33 & -5.85 & 1.44 & 159.00 & 161.00 & 0.83 & 0.19 & 0.50 & 0.97 \\ 25 \%-L & 15.00 & 9.00 & 6.67 & 3.93 & 40.00 & 40.00 & 0.89 & 0.21 & 0.47 & 0.93 \\ 25 \%-R & 15.00 & 10.00 & -5.39 & 4.56 & 40.00 & 40.00 & 0.65 & 0.21 & 0.44 & 0.79 \\ 50 \%-L-V & 14.11 & 4.36 & 2.97 & 2.17 & 79.00 & 79.00 & 0.92 & 0.21 & 0.45 & 0.93 \\ 50 \%-R-V & 15.00 & 4.20 & -6.11 & 2.30 & 79.00 & 80.00 & 0.89 & 0.20 & 0.49 & 0.88 \\ 50 \%-L-I V & 15.25 & 9.03 & 6.67 & 3.93 & 40.00 & 80.00 & 0.89 & 0.21 & 0.47 & 0.93 \\ 50 \%-R-I V & 14.93 & 9.69 & -5.39 & 4.56 & 40.00 & 81.00 & 0.65 & 0.21 & 0.44 & 0.79\end{array}$


clv_1_2-@SM-table.txt

thr \pm SE PSE \pm SE IT AT MLL SSE R2 M-Corr

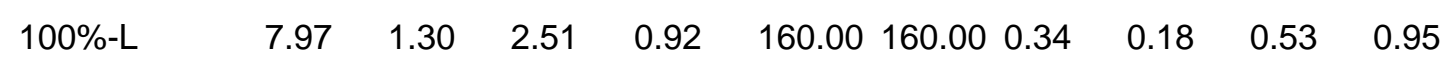

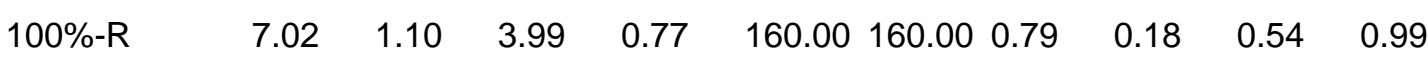

$\begin{array}{lllllllllll}75 \%-\mathrm{L} & 9.01 & 1.77 & 5.15 & 1.20 & 118.00 & 118.00 & 0.69 & 0.19 & 0.51 & 0.96\end{array}$

$\begin{array}{lllllllllll}75 \%-\mathrm{R} & 8.85 & 1.82 & 3.98 & 1.16 & 119.00 & 121.00 & 0.64 & 0.19 & 0.52 & 0.95\end{array}$

$\begin{array}{lllllllllll}50 \%-\mathrm{L} & 19.84 & 4.07 & 9.49 & 2.12 & 160.00 & 160.00 & 0.89 & 0.20 & 0.45 & 0.93\end{array}$

$\begin{array}{llllllllllll}50 \%-R & 16.32 & 3.26 & 7.44 & 1.75 & 154.00 & 160.00 & 0.49 & 0.20 & 0.45 & 0.90\end{array}$

$\begin{array}{lllllllllll}25 \%-L & 18.00 & 18.00 & 9.51 & 7.58 & 39.00 & 39.00 & 0.88 & 0.21 & 0.49 & 0.69\end{array}$

$\begin{array}{lllllllllll}25 \%-R & 22.00 & 46.00 & 6.88 & 7.25 & 40.00 & 42.00 & 0.97 & 0.23 & 0.36 & 0.92\end{array}$

$\begin{array}{lllllllllll}50 \%-L-V & 11.34 & 2.94 & 6.93 & 1.76 & 80.00 & 80.00 & 0.87 & 0.20 & 0.49 & 0.93\end{array}$

$\begin{array}{lllllllllll}50 \%-R-V & 11.88 & 3.62 & 5.10 & 2.05 & 77.00 & 80.00 & 0.41 & 0.20 & 0.45 & 0.87\end{array}$

$\begin{array}{lllllllllll}50 \%-L-I V & 18.39 & 17.89 & 9.51 & 7.58 & 39.00 & 80.00 & 0.88 & 0.21 & 0.49 & 0.69\end{array}$

$\begin{array}{lllllllllll}50 \%-R-I V & 22.22 & 45.52 & 6.88 & 7.25 & 40.00 & 80.00 & 0.97 & 0.23 & 0.36 & 0.92\end{array}$

jaw_1_6-@SM-table.txt

thr \pm SE PSE \pm SE $\quad$ IT AT MLL SSE R2 M-Corr

$\begin{array}{lllllllllll}100 \%-\mathrm{L} & 7.15 & 1.23 & -4.28 & 0.85 & 159.00 & 159.00 & 0.84 & 0.18 & 0.52 & 0.98\end{array}$

$\begin{array}{lllllllllll}100 \%-R & 10.06 & 1.81 & -3.54 & 1.07 & 158.00 & 160.00 & 0.17 & 0.19 & 0.49 & 0.91\end{array}$

$\begin{array}{lllllllllll}75 \%-\mathrm{L} & 15.61 & 3.64 & 1.57 & 2.14 & 119.00 & 119.00 & 0.81 & 0.20 & 0.46 & 0.90\end{array}$

$\begin{array}{lllllllllll}75 \%-R & 9.85 & 2.29 & -1.92 & 1.25 & 118.00 & 120.00 & 0.45 & 0.19 & 0.51 & 0.93\end{array}$

$\begin{array}{lllllllllll}50 \%-\mathrm{L} & 16.90 & 3.50 & 1.08 & 1.88 & 160.00 & 160.00 & 0.36 & 0.21 & 0.43 & 0.87\end{array}$

$\begin{array}{lllllllllll}50 \%-R & 14.57 & 2.84 & 2.09 & 1.71 & 159.00 & 160.00 & 0.65 & 0.20 & 0.46 & 0.92\end{array}$

$\begin{array}{lllllllllll}25 \%-\mathrm{L} & 47.00 & 351.00 & 7.86 & 56.68 & 40.00 & 40.00 & 0.95 & 0.25 & 0.24 & 0.56\end{array}$

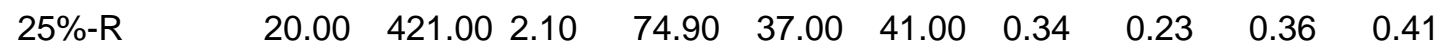

$\begin{array}{lllllllllll}50 \%-L-V & 8.87 & 2.20 & -2.34 & 1.50 & 80.00 & 80.00 & 0.58 & 0.19 & 0.51 & 0.93\end{array}$

$\begin{array}{lllllllllll}50 \%-R-V & 17.72 & 7.09 & 1.35 & 3.14 & 80.00 & 80.00 & 0.78 & 0.21 & 0.42 & 0.91\end{array}$

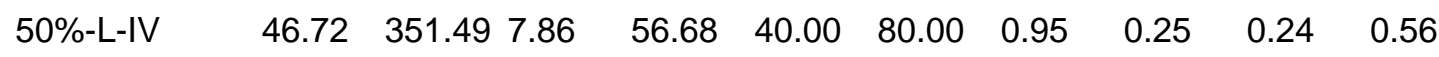

$\begin{array}{lllllllllll}50 \%-R-I V & 19.63 & 420.70 & 2.10 & 74.90 & 37.00 & 80.00 & 0.34 & 0.23 & 0.36 & 0.41\end{array}$ 
jov_1_2-@SM-table.txt

$\begin{array}{lcccccccccc} & \text { thr } & \pm S E & \text { PSE } & \pm S E & \text { IT } & \text { AT } & \text { MLL } & \text { SSE } & \text { R2 } & \text { M-Corr } \\ \text { 100\%-L } & 7.92 & 1.29 & -0.42 & 0.90 & 158.00 & 158.00 & 0.61 & 0.18 & 0.53 & 0.97 \\ 100 \%-R & 7.12 & 1.14 & -1.25 & 0.83 & 160.00 & 160.00 & 0.27 & 0.18 & 0.53 & 0.97 \\ 75 \%-L & 12.12 & 2.77 & 2.26 & 1.61 & 118.00 & 120.00 & 0.69 & 0.20 & 0.46 & 0.94 \\ 75 \%-R & 9.88 & 1.96 & -1.31 & 1.31 & 120.00 & 120.00 & 0.67 & 0.19 & 0.51 & 0.93 \\ 50 \%-L & 12.50 & 2.71 & 1.29 & 1.29 & 160.00 & 160.00 & 0.03 & 0.20 & 0.45 & 0.89 \\ 50 \%-R & 7.07 & 1.07 & -0.88 & 0.76 & 160.00 & 160.00 & 0.78 & 0.17 & 0.57 & 0.98 \\ 25 \%-L & 10.00 & 5.00 & 6.23 & 2.95 & 40.00 & 40.00 & 0.37 & 0.20 & 0.48 & 0.76 \\ 25 \%-R & 6.00 & 2.00 & -1.43 & 1.49 & 40.00 & 40.00 & 0.65 & 0.18 & 0.59 & 0.94 \\ 50 \%-L-V & 12.55 & 3.93 & 1.76 & 2.16 & 80.00 & 80.00 & 0.37 & 0.20 & 0.47 & 0.80 \\ 50 \%-R-V & 5.09 & 0.99 & -0.14 & 0.86 & 80.00 & 80.00 & 0.72 & 0.16 & 0.62 & 0.98 \\ 50 \%-L-I V & 9.57 & 4.55 & 6.23 & 2.95 & 40.00 & 80.00 & 0.37 & 0.20 & 0.48 & 0.76 \\ 50 \%-R-I V & 6.30 & 2.01 & -1.43 & 1.49 & 40.00 & 80.00 & 0.65 & 0.18 & 0.59 & 0.94\end{array}$

maf_1_7-@SM-table.txt

$\begin{array}{lcccccccccc} & \text { thr } & \pm S E & \text { PSE } & \pm S E & \text { IT } & \text { AT } & \text { MLL } & \text { SSE } & \text { R2 } & \text { M-Corr } \\ 100 \%-L & 7.83 & 1.35 & -3.62 & 0.84 & 159.00 & 159.00 & 0.76 & 0.19 & 0.50 & 0.98 \\ 100 \%-R & 8.06 & 1.38 & 2.05 & 0.89 & 160.00 & 160.00 & 0.44 & 0.19 & 0.51 & 0.96 \\ 75 \%-L & 8.73 & 1.64 & -2.24 & 1.15 & 120.00 & 120.00 & 0.76 & 0.19 & 0.50 & 0.97 \\ 75 \%-R & 15.75 & 3.82 & 2.68 & 2.08 & 120.00 & 120.00 & 0.41 & 0.21 & 0.44 & 0.88 \\ 50 \%-L & 12.97 & 2.36 & -2.20 & 1.45 & 160.00 & 160.00 & 0.76 & 0.20 & 0.45 & 0.95 \\ 50 \%-R & 13.82 & 2.58 & 4.76 & 1.57 & 160.00 & 160.00 & 0.26 & 0.20 & 0.47 & 0.86 \\ 25 \%-L & 11.00 & 8.00 & -1.33 & 3.12 & 40.00 & 40.00 & 0.99 & 0.21 & 0.47 & 0.94 \\ 25 \%-R & 16.00 & 20.00 & 7.54 & 9.58 & 40.00 & 40.00 & 0.81 & 0.21 & 0.46 & 0.92 \\ 50 \%-L-V & 11.38 & 3.85 & -4.19 & 1.89 & 80.00 & 80.00 & 0.96 & 0.20 & 0.49 & 0.97 \\ 50 \%-R-V & 14.51 & 4.59 & 1.64 & 2.46 & 80.00 & 80.00 & 0.01 & 0.21 & 0.45 & 0.68 \\ 50 \%-L-I V & 11.10 & 8.34 & -1.33 & 3.12 & 40.00 & 80.00 & 0.99 & 0.21 & 0.47 & 0.94 \\ 50 \%-R-I V & 15.86 & 20.31 & 7.54 & 9.58 & 40.00 & 80.00 & 0.81 & 0.21 & 0.46 & 0.92\end{array}$


mag_1_3-@SM-table.txt

$\begin{array}{lcccccccccc} & \text { thr } & \pm S E & \text { PSE } & \pm S E & \text { IT } & \text { AT } & \text { MLL } & \text { SSE } & \text { R2 } & \text { M-Corr } \\ 100 \%-L & 8.49 & 1.41 & -3.20 & 0.94 & 160.00 & 160.00 & 0.49 & 0.19 & 0.51 & 0.97 \\ 100 \%-R & 8.18 & 1.50 & 2.05 & 0.93 & 160.00 & 160.00 & 0.27 & 0.19 & 0.49 & 0.94 \\ 75 \%-L & 8.34 & 1.71 & -3.06 & 1.16 & 120.00 & 120.00 & 0.17 & 0.19 & 0.51 & 0.92 \\ 75 \%-R & 6.90 & 1.37 & 2.07 & 0.96 & 120.00 & 120.00 & 0.20 & 0.19 & 0.53 & 0.96 \\ 50 \%-L & 10.87 & 2.34 & -0.04 & 1.14 & 160.00 & 160.00 & 0.06 & 0.20 & 0.45 & 0.93 \\ 50 \%-R & 9.58 & 1.62 & 2.46 & 1.06 & 160.00 & 160.00 & 0.62 & 0.19 & 0.49 & 0.94 \\ 25 \%-L & 12.00 & 6.00 & -1.81 & 3.01 & 40.00 & 40.00 & 0.49 & 0.21 & 0.46 & 0.81 \\ 25 \%-R & 9.00 & 3.00 & 0.84 & 2.07 & 40.00 & 40.00 & 0.66 & 0.18 & 0.56 & 0.85 \\ 50 \%-L-V & 14.47 & 8.27 & -1.85 & 2.55 & 80.00 & 80.00 & 0.18 & 0.22 & 0.39 & 0.81 \\ 50 \%-R-V & 8.80 & 2.26 & 2.76 & 1.43 & 80.00 & 80.00 & 0.88 & 0.19 & 0.53 & 0.95 \\ 50 \%-L-I V & 11.99 & 6.44 & -1.81 & 3.01 & 40.00 & 80.00 & 0.49 & 0.21 & 0.46 & 0.81 \\ 50 \%-R-I V & 8.56 & 2.97 & 0.84 & 2.07 & 40.00 & 80.00 & 0.66 & 0.18 & 0.56 & 0.85\end{array}$

sef_1_2-@SM-table.txt

$\begin{array}{lcccccccccc} & \text { thr } & \pm S E & \text { PSE } & \pm S E & \text { IT } & \text { AT } & \text { MLL } & \text { SSE } & \text { R2 } & \text { M-Corr } \\ 100 \%-L & 7.68 & 1.45 & 2.58 & 0.88 & 160.00 & 160.00 & 0.57 & 0.19 & 0.50 & 0.96 \\ 100 \%-R & 9.90 & 1.95 & 2.52 & 1.06 & 159.00 & 160.00 & 0.81 & 0.21 & 0.44 & 0.95 \\ 75 \%-L & 6.16 & 1.09 & 6.81 & 0.87 & 120.00 & 120.00 & 0.19 & 0.17 & 0.56 & 0.97 \\ 75 \%-R & 6.45 & 1.33 & -0.73 & 0.85 & 120.00 & 120.00 & 0.55 & 0.19 & 0.52 & 0.98 \\ 50 \%-L & 8.58 & 1.38 & 3.54 & 0.94 & 160.00 & 160.00 & 0.90 & 0.18 & 0.53 & 0.98 \\ 50 \%-R & 8.69 & 1.30 & 3.44 & 0.98 & 160.00 & 160.00 & 0.98 & 0.18 & 0.53 & 0.99 \\ 25 \%-L & 6.00 & 2.00 & 5.39 & 1.42 & 40.00 & 40.00 & 0.82 & 0.18 & 0.58 & 0.97 \\ 25 \%-R & 11.00 & 4.00 & 3.61 & 2.47 & 40.00 & 40.00 & 0.67 & 0.19 & 0.54 & 0.90 \\ 50 \%-L-V & 8.54 & 2.00 & 3.61 & 1.37 & 80.00 & 80.00 & 0.74 & 0.19 & 0.53 & 0.94 \\ 50 \%-R-V & 9.86 & 2.63 & 4.16 & 1.63 & 80.00 & 80.00 & 0.89 & 0.19 & 0.51 & 0.95 \\ 50 \%-L-I V & 5.68 & 1.95 & 5.39 & 1.42 & 40.00 & 80.00 & 0.82 & 0.18 & 0.58 & 0.97 \\ 50 \%-R-I V & 10.63 & 4.03 & 3.61 & 2.47 & 40.00 & 80.00 & 0.67 & 0.19 & 0.54 & 0.90\end{array}$


sep_1_4-@SM-table.txt

$\begin{array}{lcccccccccc} & \text { thr } & \pm S E & \text { PSE } & \pm S E & \text { IT } & \text { AT } & \text { MLL } & \text { SSE } & \text { R2 } & \text { M-Corr } \\ 100 \%-L & 9.78 & 1.81 & 0.11 & 1.07 & 159.00 & 159.00 & 0.43 & 0.20 & 0.46 & 0.91 \\ 100 \%-R & 10.56 & 1.86 & 1.29 & 1.17 & 159.00 & 160.00 & 0.74 & 0.20 & 0.47 & 0.96 \\ 75 \%-L & 13.85 & 3.14 & -0.04 & 1.80 & 120.00 & 120.00 & 0.19 & 0.20 & 0.46 & 0.80 \\ 75 \%-R & 10.99 & 2.29 & -1.30 & 1.46 & 120.00 & 120.00 & 0.85 & 0.20 & 0.47 & 0.95 \\ 50 \%-L & 13.05 & 2.49 & 0.76 & 1.51 & 160.00 & 160.00 & 0.76 & 0.20 & 0.45 & 0.94 \\ 50 \%-R & 13.89 & 2.80 & 5.02 & 1.58 & 159.00 & 160.00 & 0.62 & 0.20 & 0.45 & 0.93 \\ 25 \%-L & 20.00 & 18.00 & -2.03 & 6.29 & 40.00 & 40.00 & 0.08 & 0.23 & 0.39 & 0.68 \\ 25 \%-R & 22.00 & 52.00 & 5.34 & 7.27 & 39.00 & 40.00 & 0.35 & 0.23 & 0.37 & 0.73 \\ 50 \%-\text {-V } & 13.79 & 5.43 & -0.90 & 2.28 & 80.00 & 80.00 & 0.08 & 0.22 & 0.41 & 0.76 \\ 50 \%-R-V & 15.83 & 5.38 & 4.08 & 2.72 & 79.00 & 80.00 & 0.06 & 0.21 & 0.43 & 0.70 \\ 50 \%-L-I V & 19.97 & 18.43 & -2.03 & 6.29 & 40.00 & 80.00 & 0.08 & 0.23 & 0.39 & 0.68 \\ 50 \%-R-I V & 21.90 & 52.31 & 5.34 & 7.27 & 39.00 & 80.00 & 0.35 & 0.23 & 0.37 & 0.73\end{array}$

tef_1_2-@SM-table.txt

$\begin{array}{lcccccccccc} & \text { thr } & \pm S E & \text { PSE } & \pm S E & \text { IT } & \text { AT } & \text { MLL } & \text { SSE } & \text { R2 } & \text { M-Corr } \\ 100 \%-L & 5.89 & 0.90 & -4.25 & 0.65 & 160.00 & 160.00 & 0.45 & 0.18 & 0.55 & 0.98 \\ 100 \%-R & 8.97 & 1.50 & -3.51 & 0.99 & 160.00 & 160.00 & 0.49 & 0.19 & 0.51 & 0.96 \\ 75 \%-L & 7.43 & 1.57 & -2.21 & 0.98 & 120.00 & 120.00 & 0.55 & 0.19 & 0.51 & 0.96 \\ 75 \%-R & 5.83 & 1.00 & -2.59 & 0.78 & 120.00 & 120.00 & 0.41 & 0.18 & 0.56 & 0.97 \\ 50 \%-L & 6.04 & 0.95 & -2.16 & 0.68 & 159.00 & 160.00 & 0.48 & 0.18 & 0.54 & 0.99 \\ 50 \%-R & 8.73 & 1.51 & -1.23 & 0.97 & 160.00 & 160.00 & 0.00 & 0.19 & 0.50 & 0.91 \\ 25 \%-L & 6.00 & 2.00 & -1.78 & 1.45 & 40.00 & 40.00 & 0.53 & 0.18 & 0.57 & 0.89 \\ 25 \%-R & 8.00 & 2.00 & -3.61 & 1.85 & 40.00 & 40.00 & 0.08 & 0.18 & 0.57 & 0.83 \\ 50 \%-L-V & 6.74 & 1.81 & -1.28 & 1.08 & 80.00 & 80.00 & 0.77 & 0.19 & 0.54 & 0.98 \\ 50 \%-R-V & 6.92 & 1.61 & -0.71 & 1.11 & 80.00 & 80.00 & 0.28 & 0.19 & 0.53 & 0.94 \\ 50 \%-L-I V & 6.22 & 2.25 & -1.78 & 1.45 & 40.00 & 80.00 & 0.53 & 0.18 & 0.57 & 0.89 \\ 50 \%-R-I V & 7.62 & 2.39 & -3.61 & 1.85 & 40.00 & 80.00 & 0.08 & 0.18 & 0.57 & 0.83\end{array}$




\section{Summary}

This thesis investigated the influence of the selective attention on the processing of spiral motions as member of optic flow patterns.

The main part of this thesis is the fMRI project, concentrated on examining the effect of spatial and feature-based attention on the human BOLD responses to the spiral stimuli. The fMRI study was conducted in four attention experiments. The first experiment showed a spatial attention modulation of the peak hemodynamic response to the expanding spiral in pMT and PMST. Most of the conventional neuroimaging studies reported the spatial attention in visual areas with retinotopic organization, whereas, PMST responds to both contra and ipsilateral stimulation and lacks significant retinotopic organization. Thus, the observed spatial attention modulation in PMST might add to the evidence for spatial attention modulation within the receptive fields. In the second experiment, we examined voxel-based tuning in pMT and pMST. More than $30 \%$ of the voxels in pMT and PMST showed significant tuning to spiral motions. Further, spatial attention modulated the baseline of the voxel-based tunings in pMT, but scaled up their gain in PMST. The multiplicative (stimulus-selective) modulation in PMST might indicate that spiral stimuli are more effective and preferred in MST rather than MT. In contrast, the additive (stimulus-independent) modulation in PMT might reflect the expectation of the target in the specific part of the space. In the third experiment, we assessed the effect of feature-based attention on the hemodynamic activity. We observed that the peak response decreased with attending to the same direction only in pMST but not in PMT. This area-specific feature-based attention might add to the supports for the preference of spiral motion processing in PMST rather than PMT. In the fourth experiment, we examined the feature-based attentional modulation of the voxel-based tunings in PMT and pMST. About $20 \%$ of voxels in PMT and PMST were significantly tuned to the spiral stimuli. Similar to the third experiment result, we saw gain suppression of the voxel-based tunings only in PMST. These result provided another indication for an area-specific feature-based modulation. Furthermore, these results nominate MST as a specialized area to encode and process the spiral motion patterns within the human MT-complex. Although we saw a modulation of the PMST BOLD responses in the feature-based attention experiments, the observed effects were opposing to the prediction of the feature- 
similarity gain model as the primary model of feature-based attention. Using our experimental data, we failed to determine the reason for such an inconsistency with the feature-similarity gain model. Taken together, the results of the fMRI study suggest that both spatial and feature-based attention contribute to the processing of the attended stimulus in the area with the most precedence for its encoding within the visual hierarchy.

In the second part of this thesis, we focused to investigate the effect of spatial attention on discriminating spiral motion patterns in two experiments, each with four conditions of the graded behavioral relevance. In both experiments, we altered the relevance of the target side in four grades of $25 \%, 50 \%, 75 \%$ and $100 \%$, assumed to create four attention conditions, using pre-cue with three levels of validity. In the first experiment, we only had pre-cue, while we provided subjects with a post-cue about the target location in the second experiment. The obtained results from the first experiment did not show any difference of discrimination thresholds across the four attention conditions, though; we saw that the spiral motion patterns thresholds are different across the four attention conditions in the second experiment. Moreover, we observed a linear correlation between the relevance of the target and attentional modulation in both experiments. Such an observed linear correlation between the discrimination thresholds and the attention conditions might indicate the graded allocating of the attentional recourses to the targets based on their behavioral importance.

Taken together, the results of this dissertation grant a contribution to our knowledge about the influence of attention as a cognitive factor on processing of incoming sensory information. 


\section{Bibliography}

Albright, T.D., 1984. Direction and orientation selectivity of neurons in visual area MT of the macaque. Journal of Neurophysiology 52, 1106-1130.

Albright, T.D., Desimone, R., 1987. Local precision of visuotopic organization in the middle temporal area (MT) of the macaque. Experimental Brain Research 65, 582-592.

Awh, E., Pashler, H., 2000. Evidence for split attentional foci. Journal of Experimental Psychology: Human Perception and Performance 26, 834-846.

Bashinski, H.S., Bacharach, V.R., 1980. Enhancement of perceptual sensitivity as the result of selectively attending to spatial locations. Perception \& Psychophysics $28,241-248$.

Becker, H.G., Haarmeier, T., Tatagiba, M., Gharabaghi, A., 2013. Electrical stimulation of the human homolog of the medial superior temporal area induces visual motion blindness. The Journal of neuroscience : the official journal of the Society for Neuroscience 33, 18288-18297.

Bichot, N.P., Cave, K.R., Pashler, H., 1999. Visual selection mediated by location: feature-based selection of noncontiguous locations. Percept Psychophys 61, 403423.

Bosworth, R.G., Petrich, J.A., Dobkins, K.R., 2012. Effects of spatial attention on motion discrimination are greater in the left than right visual field. Vision Research $52,11-19$.

Braun, J., Sagi, D., 1990. Vision outside the focus of attention. Perception \& Psychophysics 48, 45-58.

Brefczynski, J.A., De Yoe, E.A., 1999. A physiological correlate of the 'spotlight' of attention. Nature Neuroscience 2, 370-374.

Broadbent, D.E., 1958. Perception and Communication. Pergamon, London.

Bulakowski, P.F., Bressler, D.W., Whitney, D., 2007. Shared attentional resources for global and local motion processing. Journal of Vision 7, article 10.

Buracas, G.T., Boynton, G.M., 2007. The effect of spatial attention on contrast response functions in human visual cortex. Journal of Neuroscience 27, 93-97.

Butts, D.A., Goldman, M.S., 2006. Tuning curves, neuronal variability, and sensory coding. PLoS Biology 4, 0639-0646.

Cao, F., Hong, W., Wu, Y., Pottier, E., 2007. The Comparison of the V-Fold and the Monte-Carlo cross validation to estimate the number of clusters for the fully polarimetric sar data segmentation, Geoscience and Remote Sensing Symposium, 2007. IGARSS 2007. IEEE International, pp. 2485-2486. 
Carrasco, M., 2011. Visual attention: The past 25 years. Vision Research 51, 1484-1525.

Carrasco, M., McElree, B., 2001. Covert attention accelerates the rate of visual information processing. Proceedings of the National Academy of Sciences 98, 5363-5367.

Cook, E.P., Maunsell, J.H.R., 2002. Attentional modulation of behavioral performance and neuronal responses in middle temporal and ventral intraparietal areas of macaque monkey. Journal of Neuroscience 22, 1994-2004

Cook, E.P., Maunsell, J.H.R., 2004. Attentional modulation of motion integration of individual neurons in the Middle Temporal visual area Journal of Neuroscience 24, 7964-7977.

Coutanche, M.N., 2013. Distinguishing multi-voxel patterns and mean activation: why, how, and what does it tell us? Cogn Affect Behav Neurosci 13, 667-673.

Dale, A.M., Buckner, R.L., 1997. Selective averaging of rapidly presented individual trials using fMRI. Hum Brain Mapp 5, 329-340.

de Jong, B.M., Shipp, S., Skidmore, B., Frackowiak, R.S.J., Zeki, S., 1994. The cerebral-activity related to the visual-perception of forward motion in-depth. Brain $117,1039-1054$.

Desimone, R., Duncan, J., 1995. Neural mechanisms of selective visual attention. Annual Review of Neuroscience 18, 193-222.

Desimone, R., Ungerleider, L.G., 1986. Multiple visual areas in the caudal superior temporal sulcus of the macaque. Journal of Comparative Neurology 248, 164-189.

Dobkins, K.R., Bosworth, R.G., 2001. Effects of set-size and selective spatial attention on motion processing. Vision Research 41, 1501-1517.

Doricchi, F., Macci, E., Silvetti, M., Macaluso, E., 2010. Neural correlates of the spatial and expectancy components of endogenous and stimulus-driven orienting of attention in the Posner task. Cerebral cortex (New York, N.Y. : 1991) 20, 15741585.

Dubner, R., Zeki, S.M., 1971. Response properties and receptive fields of cells in an anatomically defined region of the superior temporal sulcus in the monkey. Brain Research 35, 528-532.

Duffy, C.J., Wurtz, R.H., 1991a. Sensitivity of MST neurons to optic flow stimuli. I. A continuum of response selectivity to large-field stimuli. Journal of Neurophysiology 65, 1329-1345.

Duffy, C.J., Wurtz, R.H., 1991b. Sensitivity of MST neurons to optic flow stimuli. II. Mechanisms of response selectivity revealed by small-field stimuli. Journal of Neurophysiology 65, 1346-1359.

Duffy, C.J., Wurtz, R.H., 1995. Response of monkey MST neurons to optic flow stimuli with shifted centers of motion. Journal of Neuroscience 15, 5192-5208. 
Duffy, C.J., Wurtz, R.H., 1997. Medial superior temporal area neurons respond to speed patterns in optic flow. Journal of Neuroscience 17, 2839-2851.

Dukelow, S.P., De Souza, J.F.X., Culham, C.J., Van den Berg, A.V., Menon, R.S., Vilis, T., 2001. Distinguishing subregions of the human MT+ complex using visual fields and pursuit eye movements. Journal of Neurophysiology 86, 1991-2000.

Eriksen, B.A., Eriksen, C.W., 1974. Effect of noise letters upon the identification of a target letter in a nonsearch task. Perception \& Psychophysics 16, 143-149.

Eriksen, C.W., St. James, J.D., 1986. Visual attention within and around the field of focal attention: A zoom lens model. Perception \& Psychophysics 40, 225-240.

Eriksen, C.W., Yeh, Y., 1985. Allocation of attention in the visual field. Journal of Experimental Psychology: Human Perception and Performance 11, 582-597.

Feldman, H., Friston, K.J., 2010. Attention, uncertainty, and free-energy. Front Hum Neurosci 4, 215.

Freeman, T.C.A., Harris, M.G., 1992. Human sensitivity to expanding and rotating motion: Effects of complementary masking and directional structure. Vision Research 32, 81-87.

Fries, P., Reynolds, J.H., Rorie, A.E., Desimone, R., 2001. Modulation of oscillatory neuronal synchronization by selective visual attention. Science 291, 1560-1563.

Fuller, S., Park, Y., Carrasco, M., 2009. Cue contrast modulates the effects of exogenous attention on appearance. Vision Res 49, 1825-1837.

Gandhi, S.P., Heeger, D.J., Boynton, G.M., 1999. Spatial attention affects brain activity in human primary visual cortex. Proceedings of the National Academy of Sciences 96, 3314-3319.

Giordano, A.M., McElree, B., Carrasco, M., 2009. On the automaticity and flexibility of covert attention: a speed-accuracy trade-off analysis. J Vis 9, 30.3110.

Gould, I.C., Rushworth, M.F., Nobre, A.C., 2011. Indexing the graded allocation of visuospatial attention using anticipatory alpha oscillations. J Neurophysiol 105, 1318-1326.

Gould, I.C., Wolfgang, B.J., Smith, P.L., 2007. Spatial uncertainty explains exogenous and endogenous attentional cuing effects in visual signal detection. $\mathrm{J}$ Vis 7, 4.1-17.

Gray, R., 2000. Attentional modulation of motion-in-depth processing. Vision Research 40, 1041-1050.

Graziano, M.S.A., Andersen, R.A., Snowden, R.J., 1994. Tuning of MST neurons to spiral motions. Journal of Neuroscience 14, 54-67. 
Hubel, D.H., Wiesel, T.N., 1968. Receptive fields and functional architecture of the monkey striate cortex. Journal of Physiology 195, 215-243.

Huk, A.C., Dougherty, R.F., Heeger, D.J., 2002. Retinotopy and functional subdivision of human areas MT and MST. Journal of Neuroscience 22, 7195-7205.

Jans, B., Peters, J.C., De Weerd, P., 2010. Visual spatial attention to multiple locations at once: the jury is still out. Psychological Review 117, 637-684.

Jefferies, L.N., Enns, J.T., Di Lollo, V., 2014. The flexible focus: Whether spatial attention is unitary or divided depends on observer goals. J Exp Psychol Hum Percept Perform 40, 465-470.

Kahnemann, D., 1973. Attention and Effort, 1st edition ed. N.J: Prentice Hall.

Kamitani, Y., Tong, F., 2005. Decoding the visual and subjective contents of the human brain. Nature Neuroscience 8, 679-685.

Kamitani, Y., Tong, F., 2006. Decoding seen and attended motion directions from activity in the human visual cortex. Current Biology 16, 1096-1102.

Karni, A., Sagi, D., 1991. Where practice makes perfect in texture discrimination: Evidence for primary visual cortex plasticity. Proceedings of the National Academy of Sciences 88, 4966-4970.

Kastner, S., De Weerd, P., Desimone, R., Ungerleider, L.G., 1998. Mechanisms of directed attention in the human extrastriate cortex as revealed by functional MRI. Science 282, 108-111.

Kastner, S., Pinsk, M.A., De Weerd, P., Desimone, R., Ungerleider, L.G., 1999. Increased activity in human visual cortex during directed attention in the absence of visual stimulation. Neuron 22, 751-761.

Kingdom, F.A.A., Prins, N., 2010. Psychophysics - A Practical Introduction. Academic Press.

Kolster, H., Mandeville, J.B., Arsenault, J.T., Ekstrom, L.B., Wald, L.L., Vanduffel, W., 2009. Visual field map clusters in macaque extrastriate visual cortex. Journal of Neuroscience 29, 7031-7039.

Kolster, H., Peeters, R., Orban, G.A., 2010. The retinotopic organization of the human middle temporal area MT/V5 and its cortical neighbors. Journal of Neuroscience 30, 9801-9820.

Komatsu, H., Wurtz, R.H., 1988a. Relation of cortical areas MT and MST to pursuit eye movements I. Localization and visual properties of neurons. Journal of Neurophysiology 60, 580-603.

Komatsu, H., Wurtz, R.H., 1988b. Relation of cortical areas MT and MST to pursuit eye movements. III. Interaction with full-field visual stimulation. Journal of Neurophysiology 60, 621-644. 
Lagae, L., Maes, H., Raiguel, S., Ziao, D.K., Orban, G.A., 1994. Responses of macaque STS neurons to optic flow components: A comparison of area MT and MST. Journal of Neurophysiology 71, 1597-1626.

Liu, T., Fuller, S., Carrasco, M., 2006. Attention alters the appearance of motion coherence. Psychonomic Bulletin \& Review 13, 1091-1096.

Liu, T., Hou, Y., 2011. Global feature-based attention to orientation. Journal of Vision 11.

Liu, T., Larsson, J., Carrasco, M., 2007. Feature-based attention modulates orientation-selective responses in human visual cortex. Neuron 55, 313-323.

Liu, T., Mance, I., 2011. Constant spread of feature-based attention across the visual field. Vision Research 51, 26-33.

Luck, S.J., Chelazzi, L., Hillyard, S.A., Desimone, R., 1997. Neural mechanisms of spatial selective attention in areas V1, V2, and V4 of macaque visual cortex. Journal of Neurophysiology 77, 24-42.

Luck, S.J., Hillyard, S.A., Mouloua, M., Hawkins, H.L., 1996. Mechanisms of visual-spatial attention: resource allocation or uncertainty reduction ? Journal of Experimental Psychology: Human Perception and Performance 22, 725-737.

Martinez-Trujillo, J.C., Treue, S., 2004. Feature-based attention increases the selectivity of population responses in primate visual cortex. Current Biology 14, 744-751.

Maunsell, J.H.R., Cook, E.P., 2002. The role of attention in visual processing. Philosophical Transactions of the Royal Society of London, Series B 357, 10631072.

Maunsell, J.H.R., Newsome, W.T., 1987. Visual processing in monkey extrastriate cortex. Annual Review of Neuroscience 10, 363-401.

Maunsell, J.H.R., Treue, S., 2006. Feature-based attention in visual cortex. Trends in Neurosciences 29, 317-322.

Maunsell, J.H.R., Van Essen, D.C., 1983a. The connections of the middle temporal visual area (MT) and their relationship to a cortical hierarchy in the macaque monkey. Journal of Neuroscience 3, 2563-2586.

Maunsell, J.H.R., Van Essen, D.C., 1983b. Functional properties of neurons in the middle temporal visual area (MT) of the macaque monkey. I. Selectivity for stimulus direction, speed, and orientation. Journal of Neurophysiology 49, 11271147.

McAdams, C.J., Maunsell, J.H.R., 1999. Effects of attention on orientation-tuning functions of single neurons in Macaque cortical area V4. Journal of Neuroscience 19, 431-441.

McCormick, P.A., Klein, R., 1990. The spatial distribution of attention during covert visual orienting. Acta Psychologica 75, 225-242. 
McMains, S.A., Somers, D.C., 2004. Multiple spotlights of attentional selection in human visual cortex. Neuron 42, 677-686.

McMains, S.A., Somers, D.C., 2005. Processing efficiency of divided spatial attention mechanisms in human visual cortex. Journal of Neuroscience 25, 94449448.

Meese, T.S., Anderson, S.J., 2002. Spiral mechanisms are required to account for summation of complex motion components. Vision Research 42, 1073-1080.

Mitchell, J.F., Sundberg, K.A., Reynolds, J.H., 2007. Differential attentiondependent response modulation across cell classes in Macaque visual area V4. Neuron 55, 131-141.

Montagna, B., Pestilli, F., Carrasco, M., 2009. Attention trades off spatial acuity. Vision Research 49, 735-745.

Moran, J., Desimone, R., 1985. Selective attention gates visual processing in the extrastriate cortex. Science 229, 782-784.

Morawetz, C., Holz, P., Baudewig, J., Treue, S., Dechent, P., 2007. Split of attentional resources in human visual cortex. Visual Neuroscience 24, 817-826.

Morrone, M.C., Tosetti, M., Montanaro, D., Florentinii, A., Cioni, G., Burr, D.C., 2000. A cortical area that responds specifically to optic flow, revealed by fMRI Nature Neuroscience 3, 1322-1328.

Motter, B.C., 1994. Neural correlates of attentive selection for color or luminance in extrastriate area V4. Journal of Neuroscience 14, 2178-2189.

Müller, H.J., Heller, D., Ziegler, J., 1995. Visual search for singleton feature targets within and across features dimensions. Perception \& Psychophysics 57, 1-17.

Murray, S.O., Wojciulik, E., 2004. Attention increases neural selectivity in the human lateral occipital complex. Nature Neuroscience 7, 70-74.

Newsome, W.T., Paré, E.B., 1988. A selective impairment of motion perception following lesions of the middle temporal visual area (MT). Journal of Neuroscience 8, 2201-2211.

O'Connor, D.H., Fukui, M.M., Pinsk, M.A., Kastner, S., 2002. Attention modulates responses in the human lateral geniculate nucleus. Nature Neuroscience 5, 12031209.

O'Craven, K.M., Rosen, B.R., Kwong, K.K., Treisman, A., Savoy, R.L., 1997. Voluntary attention modulates fMRI activity in human MT-MST. Neuron 18, 591598.

O'Craven, K.M., Savoy, R.L., 1995. Attentional modulation of activation in human MT shown with functional magnetic resonance imaging (fMRI), Association for the Research in Ophthalmology (USA), Annual Meeting, p. S856. 
Pashler, H., 1993. Doing two things at the same time. American Scientist 81, 4855.

Pashler, H., 1994. Dual-task interference in simple tasks: data and theory. Psychological Bulletin 116, 220-244.

Pastukhov, A., Fischer, L., Braun, J., 2009. Visual attention is a single, integrated resource. Vision Research 49, 1166-1173.

Pestilli, F., Carrasco, M., 2005. Attention enhances contrast sensitivity at cued and impairs it at uncued locations. Vision Research 45, 1867-1875.

Peuskens, H., Sunaert, S., Dupont, P., Van Hecke, P., Orban, G.A., 2001. Human brain regions involved in heading estimation. The Journal of neuroscience : the official journal of the Society for Neuroscience 21, 2451-2461.

Posner, M.I., 1980. Orienting of attention. Quarterly Journal of Experimental Psychology 32, 3-25.

Posner, M.I., Snyder, C.R.R., Davidson, B.J., 1980. Attention and the detection of signals. JEP-General 109, 160-174.

Richard D. Wright, L.M.W., 2008. Orienting of Attention. Oxford University Press.

Rosen, B.R., Buckner, R.L., Dale, A.M., 1998. Event-related functional MRI: Past, present, and future. Proceedings of the National Academy of Sciences 95, 773780.

Saenz, M., Buracas, G.T., Boynton, G.M., 2002. Global effects of feature-based attention in human visual cortex. Nature Neuroscience 5, 631-632.

Saenz, M., Buracas, G.T., Boynton, G.M., 2003. Global feature-based attention for motion and color. Vision Research 43, 629-637.

Saproo, S., Serences, J.T., 2010. Spatial attention improves the quality of population codes in human visual cortex. Journal of Neurophysiology 104, 885895.

Serences, J.T., 2011. Mechanisms of selective attention: response enhancement, noise reduction, and efficient pooling of sensory responses. Neuron 72, 685-687.

Serences, J.T., Boynton, G.M., 2007. Feature-based attentional modulations in the absence of direct visual stimulation. Neuron 55, 301-312.

Serences, J.T., Saproo, S., 2010. Population response profiles in early visual cortex are biased in favor of more valuable stimuli. Journal of Neurophysiology 104, 76-87.

Serences, J.T., Saproo, S., Scolari, M., Ho, T., Muftuler, L.T., 2009. Estimating the influence of attention on population codes in human visual cortex using voxelbased tuning functions. Neuroimage 44, 223-231. 
Sheinberg, D.L., Logothetis, N.K., 2001. Noticing familiar objects in real world scenes: the role of temporal cortical neurons in natural vision. Journal of Neuroscience 21, 1340-1350.

Shih, S.I., Sperling, G., 1996. Is there feature-based attentional selection in visual search? Journal of Experimental Psychology: Human Perception and Performance 22, 758-779.

Smith, A.T., Singh, K.D., Greenlee, M.W., 2000. Attentional suppression of activity in the human visual cortex. NeuroReport 11, 271-277.

Smith, A.T., Wall, M.B., Williams, A.L., Singh, K.D., 2006. Sensitivity to optic flow in human cortical areas MT and MST. European Journal of Neuroscience 23, 561569.

Somers, D.C., Dale, A.M., Seiffert, A.E., Tootell, R.B.H., 1999. Functional MRI reveals spatially specific attentional modulation in human primary visual cortex. Proceedings of the National Academy of Sciences 96, 1663-1668.

Sperling, G., Melchner, M.J., 1978. The attention operating characteristic: Examples from visual search. Science 202, 315-318.

Spitzer, H., Desimone, R., Moran, J., 1988. Increased attention enhances both behavioral and neuronal performance. Science 240, 338-340.

Steiner, V., Blake, R., Rose, D., 1994. Interocular transfer of expansion, rotation, and translation motion aftereffects. Perception 23, 1197-1202.

Stoppel, C.M., Boehler, C.N., Strumpf, H., Heinze, H.J., Noesselt, T., Hopf, J.M., Schoenfeld, M.A., 2011. Feature-based attention modulates direction-selective hemodynamic activity within human MT. Human Brain Mapping 32, 2183-2192.

Takeuchi, T., 1997. Visual search of expansion and contraction. Vision Research 37, 2083-2090.

Tanaka, K., Saito, H.-A., 1989. Analysis of motion of the visual field by direction, expansion/contraction, and rotation cells clustered in the dorsal part of the medial superior temporal area of the macaque monkey. Journal of Neurophysiology 62, 626-641.

Thomas Yeo, B.T., Krienen, F.M., Sepulcre, J., Sabuncu, M.R., Lashkari, D., Hollinshead, M., Roffman, J.L., Smoller, J.W., Zollei, L., Polimeni, J.R., Fischl, B., Liu, H., Buckner, R.L., 2011. The organization of the human cerebral cortex estimated by intrinsic functional connectivity. J Neurophysiol 106, 1125-1165.

Tootell, R.B., Mendola, J.D., Hadjikhani, N.K., Liu, A.K., Dale, A.M., 1998a. The representation of the ipsilateral visual field in human cerebral cortex. Proceedings of the National Academy of Sciences of the United States of America 95, 818-824.

Tootell, R.B., Taylor, J.B., 1995. Anatomical evidence for MT and additional cortical visual areas in humans. Cerebral cortex (New York, N.Y. : 1991) 5, 39-55. 
Tootell, R.B.H., Hadjikhani, N., Hall, E.K., Marrett, S., Vanduffel, W., Vaughan, J.T., Dale, A.M., 1998b. The retinotopy of visual spatial attention. Neuron 21, 1409-1422.

Treue, S., 2001. Neural correlates of attention in primate visual cortex. Trends in Neurosciences 24, 295-300.

Treue, S., Martinez-Trujillo, J.C., 1999. Feature-based attention influences motion processing gain in macaque visual cortex. Nature 399, 575-579.

Treue, S., Martinez-Trujillo, J.C., 2012. The spotlight of attention: shifting, resizing and splitting receptive fields when processing visual motion. e-Neuroforum 3, 7479.

Treue, S., Maunsell, J.H.R., 1996. Attentional modulation of visual motion processing in cortical areas MT and MST. Nature 382, 539-541.

Treue, S., Maunsell, J.H.R., 1999. Effects of attention on the processing of motion in macaque middle temporal and medial superior temporal visual cortical areas. Journal of Neuroscience 19, 7591-7602.

Ungerleider, L.G., Desimone, R., 1986. Projections to the superior temporal sulcus from the central and peripheral field representations of V1 and V2. Journal of Comparative Neurology 248, 147-163.

Van Essen, D.C., Maunsell, J.H.R., 1983. Hierarchical organization and functional streams in the visual cortex. Trends in Neurosciences, 370-375.

Verghese, A., Anderson, A.J., Vidyasagar, T.R., 2013. Space, color, and direction of movement: how do they affect attention? J Vis 13.

Verghese, P., Kim, Y.J., Wade, A.R., 2012. Attention selects informative neural populations in human v1. Journal of Neuroscience 32, 16379-16390.

Wall, M.B., Lingnau, A., Ashida, H., Smith, A.T., 2008. Selective visual responses to expansion and rotation in the human MT complex revealed by functional magnetic resonance imaging adaptation. European Journal of Neuroscience 27, 2747-2757.

Yeshurun, Y., Carrasco, M., 1998. Attention improves or impairs visual performance by enhancing spatial resolution. Nature 396, 72-75.

Yeshurun, Y., Carrasco, M., 1999. Spatial attention improves performance in spatial resolution tasks. Vision Research 39, 293-306.

Zeki, S., 1991. Cerebral akinetopsia (visual motion blindness). A review. Brain 114 ( Pt 2), 811-824.

Zeki, S., Watson, J.D.G., Lueck, C.J., Friston, K.J., Kennard, C., Frackowiak, R.S.J., 1991. A direct demonstration of functional specialization in human visual cortex. Journal of Neuroscience 11, 641-649. 
Zeki, S.M., 1974. Functional organization of a visual area in the posterior bank of the superior temporal sulcus of the rhesus monkey. Journal of Physiology 236, 549-573.

Zizlsperger, L., Sauvigny, T., Haarmeier, T., 2012. Selective attention increases choice certainty in human decision making. PLoS One 7, e41136. 


\section{Curriculum Vitae}

\section{Sepideh Fazeli Neishabour}

\section{Personal Information:}

- Date of Birth: April 20, 1983

- Gender: female

\section{Education:}

- $\mathrm{PhD}$. Candidate in Systems Neuroscience, Graduate School for Neurosciences and Molecular Biosciences (GGNB), Georg-August University, Göttingen, Germany, Since May 2010.

- M.Sc. in Electrical Engineering, Bioelectric, University of Tehran, Tehran, Iran, 2006-2009.

Total GPA: 17.06/20.

Thesis: "Computational Modeling of Language Acquisition based on a Connectionist Approach"

Supervisor: Dr. Fariba Bahrami, Co-advisors: Prof. Caro Lucas and Prof. Reza Nilipour

Thesis Score: Excellent

- B.Sc. in Electrical Engineering, Communication, Ferdowsi University, College of Engineering, Mashhad, Iran, 2001-2006. Total GPA: 16.87/20.

Thesis: "Implementation of a Model-Based 3D Tracking System for Augmented Reality"

Supervisor: Dr. Hamidreza Pourreza

Thesis Score: Excellent

- Pre-University Certificate, Farzanegan High School - NODET (National Organization for Development of Exceptional Talents), Mashhad, Iran, 2001.

- High school Diploma in mathematics, Farzanegan High School - NODET (National Organization for Development of Exceptional Talents), Mashhad, Iran, 1997-2000.

\section{Research Interests:}

Neural Imaging, Computational Neuroscience, Cognitive Neuroscience.

Modeling, Data Analysis, Pattern Recognition.

Neural Networks, Learning, Artificial Intelligence.

\section{Honors and Awards:}


Sepideh Fazeli, Carsten Schmidt-Samoa, Peter Dechent, Stefan Treue, "Attentional modulation of human BOLD responses to spiral motion patterns, ” in preparation

Sepideh Fazeli, Stefan Treue, "Graded visual attention in discriminating human spiral motion patterns," in preparation

Sepideh Fazeli, Carsten Schmidt-Samoa, Peter Dechent, Stefan Treue, "Feature-based attentional modulation of human BOLD responses to spiral motion patterns," Society for Neuroscience, Annual Meeting, San Diego, CA, November 2013.

Sepideh Fazeli, Carsten Schmidt-Samoa, Peter Dechent, Stefan Treue, "Population tuning and attentional modulation of human BOLD response to spiral motion patterns, " German Society for Neuroscience Meeting, Göttingen, March 2013.

Sepideh Fazeli, Carsten Schmidt-Samoa, Peter Dechent, Stefan Treue, "Attentional modulation of human BOLD response in processing of spiral motion patterns, " Society for Neuroscience, Annual Meeting, New Orleans, LA, October 2012.

Sepideh Fazeli, Anselm Rothe, Stefan Treue, "Attentional modulation of human spiral motion discrimination," 9th Göttingen Meeting of the German Neuroscience Society, Göttingen, Germany, March 2011.

Sepideh Fazeli, Fariba Bahrami, "A computer model of language acquisition - syllable learning - based on Hebbian cell assemblies and reinforcement learning," International Conference on Computer Science and Engineering, ICCSE 2009, Dubai, United Arab Emirates, January 2009.

Sepideh Fazeli, Fariba Bahrami, "A computer model of sequence learning during language acquisition," Accepted in 15th International Conference on Neural Information Processing, ICONIP 2008, Auckland, New-Zealand, November 2008.

Sepideh Fazeli, Maryam Naghibolhosseini, Fariba Bahrami, "An adaptive neuro-fuzzy inference system for diagnosis of aphasia”, 2nd International Conference on Bioinformatics and Biomedical Engineering, iCBBE 2008, Shanghai, China, May 2008.

Maryam Naghibolhosseini, Amir-Homayoun Jafari, Sepideh Fazeli, Caro Lucas, "Fuzzy clustering of transient evoked otoAcoustic emission signals using Gustafson Kessel algorithm, " 2nd International Conference on Bioinformatics and Biomedical Engineering, iCBBE 2008, Shanghai, China, May 2008.

Maryam Naghibolhosseini, Amir-Homayoun Jafari, Sepideh Fazeli, Saeed Mahmoudian, "Fuzzy clustering of transient evoked otoAcoustic emission signals based on K-Nearest neighbors rule," 6th IASTED International Conference on Biomedical Engineering, BioMED2008, Innsbruck, Austria, February 2008.

H.R. Pourreza, M. Ameli, A.M .Nayebi, S. Fazeli, B. Taghizadeh, "Automatic detection of eggshell defects based on image processing," 4th International Conference on Integrated Systems for AGRI-FOOD Production, SIPA'05, Timisoara, Romania, November 2005. 
Tuebingen spring school: methods to study the brain in action, Tuebingen, Germany, March 2012.

6th M-BIC fMRI workshop, Maastricht, Netherlands, March 2011.

2nd G-Node winter course in neural data analysis, Muenchen, Germany, March 2010.

Summer school on computational vision, Rauischholzhausen, Germany, August 2009.

Functional imaging graduate course, Fall 2007. Course project: A comprehensive lecture on resting state connectivity

Digital signal processing graduate course, Fall 2007. Course project: Implementation of a demosaicking algorithm using wavelet transforms

Pattern recognition graduate course, Spring 2006. Course project: Implementation and evaluation of several methods for aphasia diagnosis and presenting a novel approach for a better diagnosis

Biological system modeling graduate course, Spring 2006. Course project: Implementation of a neuronal model of language cortex for word learning

System identification graduate course, Spring 2006. Course project: Implementation of a monocular model-based 3D tracking system using extended Kalman filter

Digital image processing graduate course, Fall 2006. Course project: Implementation of a locally constrained watershed transform

Bioinstruments graduate course, Fall 2006. Course project: A Comprehensive lecture on designing glucometers \& telemedicine

\section{Research and Working Experience:}

"German Primate Center (DPZ), Cognitive Neuroscience Lab (AKN)", research assistant on the electrophysiology project of "saccade and reward in dorsal stream of macaques" under supervision of Prof. Stefan Treue, Goettingen, Germany, 2009-2010.

"Center of Excellence of Information and Communication Technology (CEICT)", research assistant on the national project of "model for communication and ICT sectors in IRAN liberalization" under Supervision of Prof. Caro Lucas and Prof. Hassan Shanechi. Tehran, Iran, 2006-2007.

"Bina Pardaz Co.", research assistant on the project of "design and implementation of a virtual studio tracking system" proposed and approved in Islamic Republic of Iran Broadcasting (IRIB), under supervision of Dr. Hamidreza Pourreza, Mashhad, Iran, 20052006. 
"Bina Pardaz Co.", research assistant on the project of "design of an automatic eggshell defect detector system" proposed and approved in Islamic Azad University, under supervision of Dr. Hamidreza Pourreza, Mashhad, Iran, 2004-2005.

"Digital Signal Processors Lab", design and implementation of TMS320c25 minimum board, under supervision of Dr. Alireza Seyedein, Ferdowsi University, College of Engineering, Mashhad, Iran, 2004-2005.

"Bina Pardaz Co.”, Industrial Trainee, Mashhad, Iran, 2004.

\section{Computer and Programming Skills:}

Software: MATLAB, Brain Voyager, PRESENTATION, R Statistics

Programming Language: C, Basic, Visual Basic

\section{Additional Professional Activities:}

Working with TI-Digital Signal Processors (TMS320c20)

Working with microcontrollers, 80C5x series

\section{Languages:}

Farsi (Native), English (Fluent), German (Intermediate), Arabic (Basic) 